\title{
Forty years of antiprotons
}

\section{J. Eades}

\author{
CERN, 1211 Geneva 23, Switzerland
}

\section{F. J. Hartmann}

Physik-Department, Technische Universität München, D-85747 Garching, Germany

The discovery of the antiproton some 40 years ago and the almost synchronous fall of parity $(P)$ and charge conjugation $(C)$ symmetries were soon followed by the realization that $C P T$ rather than $C$ invariance is the fundamental symmetry connecting matter and antimatter, and that consequently any measurement of the antiproton's properties can be interpreted as a test of that symmetry. It is the latter view of the antiproton, as an object of study in its own right, rather than as a means to such other ends as the production of gauge bosons and meson resonances, that is presented here. The authors review the technical steps that have led from the handful of antiprotons observed by Chamberlain, Segrè, Wiegand, and Ypsilantis to the intense, high-quality beams available today and show how the state of rest and isolation required for high precision measurements of their properties can be achieved by confining them in electromagnetic traps or in their microscopic counterparts, exotic atoms. The test bench role of antiprotons and antihydrogen atoms for both $C P T$ symmetry and the gravitational weak equivalence principle is discussed, and the body of experimental results obtained since 1955 critically reviewed from this standpoint. Future experiments are then discussed in the light of the closure of the CERN Low Energy Antiproton Ring (LEAR), its replacement in 1999 by the Antiproton Decelerator (AD), and the likely antiproton source at the Japan Hadron Facility. [S0034-6861(99)00401-8]

\section{CONTENTS}

I. Introduction

A. Historical note

B. The antiproton at 40 and beyond

C. Scope of this review

II. Producing and Accumulating Antiprotons

A. Production methods

B. Accumulating antiprotons

III. Decelerating GeV Antiprotons

A. From $\mathrm{GeV}$ to $\mathrm{MeV}$

1. LEAR

2. The Antiproton Decelerator, AD

B. Deceleration below $1 \mathrm{MeV}$

1. Passive energy dissipation

2. The cyclotron trap, or "anticyclotron"

3. Radio-frequency quadrupoles

IV. Practical Conditions of Rest and Isolation

A. Macroscopic traps

1. Loading antiprotons into traps

2. Motion in traps

3. Deceleration and cooling in traps

B. Microscopic traps: exotic atoms

1. Slowing down below the Bragg peak

2. The formation of antiprotonic atoms

3. The structure of antiprotonic atoms

4. The antiprotonic atom cascade

5. The metastable antiprotonic helium atom as antiproton trap

C. Confinement, cooling, and isolation of antihydrogen atoms

1. Neutral-atom traps

2. Atomic beams

V. Fundamental Symmetries, Antiparticles, and Antimatter

A. Antiprotons, antihydrogen, and the $C P T$ theorem

B. Antiprotons, antihydrogen, and the weak-equivalence principle

VI. Studies of Antiprotons and Antihydrogen Atoms to December 1996

397

A. Direct measurements

1. Direct measurements of mass

2. Direct measurement of lifetime

3. Direct measurement of charge-bulk matter method

4. Direct measurement of the magnetic moment in traps

B. Indirect measurements

1. Cyclotron frequencies and mass/charge determinations

2. Exotic atoms: The antiprotonic Rydberg and mass/charge determinations

3. Exotic atoms and the antiproton charge

4. Magnetic moment from exotic atoms

5. Lifetime from the antiproton abundance in cosmic radiation

C. Antiproton ballistics

D. Experiments with "hot" antihydrogen

VII. Physics with Cold Antihydrogen

A. Positron sources

B. Recombination reactions

C. Spectroscopy

D. Evaluation of antihydrogen results in terms of $C P T$

E. Evaluation of antihydrogen results in terms of gravitation

VIII. Experiments with the CERN AD

A. Cold antihydrogen: production and spectroscopy

B. Antiprotonic helium and protonium

IX. Concluding Remarks-The Road Ahead

Acknowledgments

References

\section{INTRODUCTION}

395

395

396

\section{A. Historical note}

It is now more than forty years since the November 1955 announcement of the discovery of the antiproton 
by Chamberlain, Segrè, Wiegand, and Ypsilantis. The immediate context of the search for the antiproton was that while the positron had been clearly identified many years before as the charge conjugate or Dirac counterpart of the electron, it was still possible to question the assumption that the proton was a fundamental Dirac particle at all; its extremely large anomalous magnetic moment, one of the puzzles of the time (Ypsilantis, 1996, 1997), suggested that the Dirac equation might not give a complete description of it.

When looking at the experiment as a piece of scientific history, it is impossible not to be struck by the near contemporaneity of two other far-reaching developments in physics - the observation of parity violation in weak interactions and the establishment of the $C P T$ theorem as the most fundamental among discrete fundamental symmetries.

By April 1958, when Segrè completed the literature survey for his review article on antinucleons in Volume 8 of the Annual Review of Nuclear Science (1958), it had become evident that the parity $(P)$ violation experiments carried out by $\mathrm{C}$. S. Wu and her colleagues in December 1956 (Wu et al., 1957) had also invalidated $C$ invariance as a general property of all interactions (Lee and Yang, 1956), although no conclusion could be drawn at the time about the invariance or violation of $T$ symmetry (i.e., motion reversal). The $C$ and $P$ violations were at the one-part-in- $10^{7}$ scale characteristic of weak interactions. If $C$ invariance breaks down, how could the equality of the masses and lifetimes of any particle/ antiparticle pair be maintained $?^{1}$ From considerations of all data available from the antiproton experiment, Segrè was able to establish the equality of the antiproton and proton masses at the $2 \%$ level, and a lower limit on the antiproton lifetime of $10^{-7} \mathrm{~s}$ could be deduced from the fact that it survived long enough to reach the end of the experimental beamline. ${ }^{2}$ The contemporary figures (Crowe, 1957) for the $\mu^{+}$and $\mu^{-}$leptons were that the masses were equal to $\sim 0.1 \%$ and the lifetimes to $\sim 1 \%$.

The principle that clarified all these matters was of course the $C P T$ theorem. Left untouched by the revolution introduced by $C$ and $P$-violation experiments the theorem had, by 1956, been shown by Bell (1955), Pauli (1955), and Lüders $(1954,1956)$ to represent the minimal set of conditions for the existence of any relativistic field theory consistent with both quantum mechanics and special relativity. ${ }^{3}$ What now became apparent was that $C P T$ invariance alone (Lüders and Zumino, 1957; Lee, Oehme, and Yang, 1957), and not the stronger condition of $C$ invariance, was sufficient to guarantee the equality of the masses, lifetimes, charges, spins, and

\footnotetext{
${ }^{1}$ The point in question was not the weakness or strength of interactions, but that $C$ invariance was at the time accepted as the reason for the very existence of antiparticles.

${ }^{2}$ See Sec. VI.A. for values and methods.

${ }^{3}$ The best-known of Lüders' papers on the theorem is the one appearing in Annals of Physics (Lüders, 1957) a little later.
}

magnetic moments of particle-antiparticle pairs, although as Lüders and Zumino pointed out, not in general of branching ratios.

Astrophysicists were not slow to speculate on the cosmic significance of the presence or absence of antimatter in the universe, or of possible anomalies in its gravitational properties. Segrè (1958) and Morrison (1958) both made the point, sometimes forgotten even now, that it is the weak-equivalence principle, and not the $C P T$ theorem that requires the gravitational masses of protons and antiprotons to be the same, although these authors realized that any difference would give rise to quite obvious complications in general relativity. ${ }^{4}$ Burbidge and Hoyle $(1956,1957)$ estimated a maximum concentration of antimatter in our galaxy of $10^{-7}$, interpreting this figure in terms of the steady state, or continuous creation model that was then the favored cosmological candidate.

All these developments were part of the general emergence of the idea that, as Park (1958) so neatly put it, "the degree of assurance that nature exhibits a certain symmetry is essentially furnished by the precision of the experiments which support the corresponding conservation law." Indeed, interactions in general might be severely enough constrained by the invariance principles associated with such symmetries to be wholly determined by them. ${ }^{5}$ In any case it would be wise in future to relegate a priori assumptions like universal left-right symmetry to the status of aesthetic preferences, not necessarily shared by Nature and therefore in need of rigorous testing by experiment.

Considerations like those of Park simply demonstrate the falsifiability criterion of Karl Popper (1959) in action: a conservation law that has survived scrutiny at a certain level of precision is not guaranteed against falsification by yet more precise tests. It was then promising to think of all measurements of the properties of antiparticles in this light. Rather than being an embarrassment in the face of $C$-violating weak interactions, the equal masses and lifetimes of lepton/antilepton and baryon/antibaryon pairs could be interpreted as experimental checks on the weaker but more general $C P T$ symmetry, although with a very low degree of assurance given the scale of $P$ and $C$ violations. Finally, investigations of the gravitational properties of antiparticles and/or antiatoms could be seen as a means of furnishing an additional degree of assurance concerning general relativity, but in a new domain - that of antimatter. Evi-

\footnotetext{
${ }^{4}$ It has been pointed out by Okun $(1989,1992)$ that general relativity makes the distinction between inertial and gravitational mass redundant for slow particles and wrong for fast ones, as the "charge" on which gravitational forces pull is the particle's energy-momentum tensor and not its mass. For a slow particle in a weak field the tensor reduces to a single element which determines both its inertial properties and its behavior under gravity.

${ }^{5}$ This argument that full recognition of the symmetry of a physical problem is tantamount to its complete dynamical solution has been discussed by Kabir $(1965,1995)$.
} 
dently though, the measurement precision for the static properties of antiprotons would have to be improved by many orders of magnitude if they were to seriously challenge either $C P T$ or general relativity in the Popperian sense.

At what level, then, might $C P T$ or other discrete symmetry violations begin to appear? The $10^{-7}$ scale of $C$ and $P$ violations gave some rough indication that, following the logic of Park, any further violations would certainly have to be sought at a still smaller level than $10^{-7}$-one might guess at perhaps at $10^{-14}$ - the next order in the weak-interaction coupling constant. ${ }^{6}$

But how could the impediment of the proton's large anomalous moment noted in the first paragraph of this review be removed? As we shall see in Sec. V.A, however imperfectly the internal structure of the proton was understood in 1956, its constituent Dirac particles would have to interact in such a way as to produce a $C P T$-symmetrical antiproton. Even before the advent of quantum chromodynamics, therefore, the anomalous moment had ceased to be a mystery. ${ }^{7}$

The discovery of the antiproton was therefore not only a new and direct confirmation of the Dirac equation (its relation to the $C P T$ theorem made clear for once and for all), but an implicit challenge to experimentalists to probe the foundations of modern physics still further by comparing its properties closely with those of the proton. Indeed, it is arguable whether measurements of the antiproton's properties have any other merit, as $C P T$ symmetry alone tells us what they should be.

\section{B. The antiproton at 40 and beyond}

Looking at the situation in 1997, we must admit that this challenge has not yet been fully met. The span of some forty years between 1956 and the present is as great as that between J. J. Thomson's discovery of the electron and the Lamb-Retherford observation of the minute $2 S_{1 / 2}-2 P_{1 / 2}$ energy-level difference in the hydrogen atom that brought the first experimental confirmation of QED (or, what Popper would consider more relevant, the first falsification of the Dirac equation). Yet as late as August 1978 the empirical lower limit on the antiproton lifetime could only be set at $120 \mu$ s (Evans et al., 1989); it was only in 1988 that its magnetic moment was determined with a precision of better than one percent (Kreissl et al., 1988), only in 1990 that the experimental constraint on its $e / m$ ratio was pushed below the level (modest even at the $P$-violation scale) of one part in a million (Gabrielse et al., 1990), and only in 1996 that the first atoms of antihydrogen were produced

\footnotetext{
${ }^{6}$ When $C P$ violation was finally found in 1963 , it did indeed appear at this $10^{-14}$ scale.

${ }^{7}$ We have to remember here that, the $C P T$ theorem notwithstanding, it was only in the late 1970 s that the successes of quantum chromodynamics made relativistic quantum field theories a respectable topic in polite company (Zichichi, 1996).
}

(Baur et al., 1996). And forty years after Segrè's conclusion that "the question of the gravitational properties of antimatter can ultimately only be resolved by experiment," we are still waiting for that experiment to be done.

The reason for the absence of measurements on the antiproton of equivalent precision to corresponding measurements on the proton is that really high-precision experiments on any particle (or atom) require it to be both isolated and at rest. Isolation from the perturbing effects of collisions is an obvious prerequisite to any close study of a given particle's properties, while the state of rest in the laboratory is, in general, the only condition under which it can be kept under observation for long periods. For the electron and proton, this condition has best been approached by confining them in potential wells, easily realizable in the form of electromagnetic-field configurations that pull the electrical charge of these particles towards some central point or region of stability; such configurations are known as charged-particle traps and figure extensively in the discussions that follow. Even when these two particles are bound together as a hydrogen atom, achieving the highest precision in atomic spectroscopy requires that this atom be kept still in a laser beam, either by the use of neutral-atom traps that pull the electron magnetic moment (and therefore the whole atom) to a central equilibrium point, or by confining the atoms in a cold atomic beam that stays within the region covered by a collinear laser probe.

When we turn to precision studies of the antiproton and the positron, and of their bound state, the antihydrogen atom, we must clearly aim at the same ideal condition of rest and isolation. However, not only is the isolation problem now augmented considerably by the phenomenon of annihilation, but the state of rest is itself far more difficult to achieve since both particles must necessarily be produced from high-energy sources. Many of the technical challenges of such studies are associated with achieving (or at least approaching) this ideal state, and we shall need to return to this point many times below. The problem is alleviated to some extent for the antiproton, as its negative charge allows it to be confined very easily in the potential well surrounding ordinary atomic nuclei. Such naturally occurring traps are more commonly known as exotic atoms. They are very easily formed but, with one important exception, they do not buy the antiproton much time against annihilation.

These techniques of decelerating, trapping, and manipulating charged particles are currently under rapid development, and it is therefore not surprising that the antiproton (together with the positron) has at last begun to assume a central place in the study of fundamental physics. ${ }^{8}$ Furthermore, parallel developments in hydrogen atom traps (Cesar, 1997) and in high-precision laser spectroscopy (Pachucki et al., 1996; Adams and Riis,

\footnotetext{
${ }^{8}$ This is perhaps best indicated by the value obtained for the antiproton's $e / m$ ratio, within $1.5 \times 10^{-9}$ of that of the proton, by Gabrielse et al. (1990); it is discussed in Sec. VI.B.1.
} 
1997) suggest that the antihydrogen atom can soon play a role in the study of the antiworld similar to that played by the hydrogen atom over more than a century of scientific history. Very recently, indeed, it has been decided to construct at CERN a new experimental facility for just such a program of research. This Antiproton Decelerator (AD) will replace the now-defunct CERN LowEnergy Antiproton Ring (LEAR), with which much of our meager experience of the antiworld has been associated.

\section{Scope of this review}

It is therefore largely (but not exclusively) to the practical role of the antiproton as a high-precision probe of $C P T$ invariance and of the gravitational properties of antiparticles that we turn our attention in this review. The field is wide enough for a book rather than an article, and we have been obliged to omit certain secondary topics. Thus we do not discuss the use of the antiproton to label $K^{0}$ and $\overline{K^{0}}$ in measurements of $K^{0} \overline{K^{0}}$ $C P$-violation parameters, nor the suggested search (Kabir, 1995) for atomic $C P$ violation by comparisons of parity-violating effects (such as $S_{1 / 2}-P_{1 / 2}$ mixing) in hydrogen and antihydrogen, nor nuclear antimatter as embodied in the antideuteron and antitriton. In the topics we do discuss, we have tried to follow a pedagogical style throughout in order to make the review in its entirety accessible to graduate students and workers in other fields. We direct the reader who wishes to dig deeper to other, more detailed reviews of individual topics only briefly summarized here. Some of these are, for antihydrogen, Charlton et al. (1994) and Greenland (1997); for ion traps, particularly for antiprotons, Brown and Gabrielse (1986); for CPT and other invariance principles, Kabir (1965); and for gravitational interactions with the electromagnetic field, Thirring (1961, 1969); others can be found in the reference list.

The first problems to be addressed are the technical ones discussed in Secs. II-IV: how to produce antiprotons and collect a sample of them; how to bring this sample to rest, given that Nature has insisted on production mechanisms that give its members a kinetic energy of at least $1 \mathrm{GeV}$; and finally how are we then to isolate the sample from ordinary matter?

Having indicated how to prepare our raw material, we return in Sec. V to the question raised briefly above of why it is important to carry out $C P T$ and weakequivalence principle (WEP) tests with it.

Section VI is devoted to an evaluation of experiments on the physics of the antiproton obtained prior to the closure of LEAR in December 1996, although it is not confined to results obtained with that machine. This section is brought to a topical end with a discussion of the recent experiments on the production at CERN and Fermilab of "hot" antihydrogen atoms. We then turn (Sec. VII) to the greater difficulties of producing, and the commensurately greater promise of studying, cold ones, ending with a critical evaluation of antihydrogen as both a $C P T$ and a WEP laboratory.
This is followed (Sec. VIII) by a look ahead at what is likely to be the future of antiproton and antimatter research in the era of the CERN Antiproton Decelerator, and by a brief mention of possibilities at the future Japanese Hadron Facility. Section IX presents our conclusions in the light of these developments.

\section{PRODUCING AND ACCUMULATING ANTIPROTONS}

\section{A. Production methods}

The Berkeley Bevatron, which produced the first handful of antiprotons seen in the world, was completed in 1954 and had a maximum proton kinetic energy of 6.2 $\mathrm{GeV}$. At threshold, by definition, antiprotons are produced at rest in the center-of-mass system, and conservation of nucleon number further requires two new proton masses to be added to the two old ones. The laboratory threshold energy for producing antiprotons by directing a proton beam onto a stationary proton target is then exactly $6 m_{p}$, as these four equal-mass particles must each emerge in the laboratory with one quarter of the beam momentum. In fact, the target used in the experiment of Chamberlain et al. (1957) was not hydrogen but copper, so that Fermi motion of protons within the target nuclei reduced the threshold a little. The authors also produced an excitation function showing the number of antiprotons per $10^{5} \pi$-rising from zero at $4.25 \mathrm{GeV}$ to 2.3 at $6.2 \mathrm{GeV}$.

In order to produce antiprotons in large numbers it would obviously be necessary to go far above threshold. Under such conditions, the antiprotons, no longer at rest in the center-of-mass system, would be boosted by the Lorentz transformation to laboratory energies far beyond the threshold value of $\approx 1 \mathrm{GeV}$ and be taken still further away from the ideal condition of rest necessary for precision measurements.

Several fountains of antiprotons have come and gone in the world since 1954. Among these the one at CERN may serve as an example. With an energy of $26 \mathrm{GeV} / c$ and a proton-beam intensity on the order of $10^{13} \mathrm{~s}^{-1}$ the CERN proton synchrotron (PS) beam can produce antiprotons abundantly enough for typical high-energy particle-physics experiments, in which the cross sections to be studied are of order $\mu b$ or $n b$, although the antiprotons have for some time mainly been used for experiments below the 1-GeV threshold energy. Möhl (1997) summarizes the general features of the CERN system, with particular reference to this low-energy context. At Fermilab and elsewhere the production schemes are quite similar but remain fully oriented towards the needs of high-energy experiments, although this situation may soon change (Jackson, 1997). A full and excellent description of both the CERN and the Fermilab sources has been provided by Church and Marriner (1993).

The beam from the CERN proton synchrotron is extracted onto an iridium target, typically a cylinder of 3 $\mathrm{mm}$ diameter and $50 \mathrm{~mm}$ length, embedded in graphite. A useful parametrization of the inclusive cross section 
TABLE I. Empirical target-dependent parameters of Hojvat and Van Ginneken (1983).

\begin{tabular}{cccc}
\hline \hline Target & $\mathrm{a}$ & $\mathrm{b}$ & $\mathrm{c}$ \\
\hline $\mathrm{H}$ & 1.00 & 0.00 & 0.00 \\
$\mathrm{Be}$ & 0.90 & 0.95 & 0.61 \\
$\mathrm{Al}$ & 1.22 & 1.15 & 0.87 \\
$\mathrm{Cu}$ & 1.69 & 1.38 & 1.79 \\
$\mathrm{~W}$ & 1.5 & 1.43 & 1.56 \\
$\mathrm{~Pb}$ & 1.73 & 1.37 & 1.83 \\
\hline \hline
\end{tabular}

for antiproton production has been derived by Hojvat and van Ginneken (1983) from experimental data on a number of targets:

$$
\begin{aligned}
\left(E / \sigma_{\mathrm{abs}}\right)\left(d^{2} \sigma / d p_{t} d p_{z}\right)= & {\left[0.065\left(1-x_{r}\right)^{8} \exp \left(-3 p_{t}^{2}\right)\right] } \\
& \times\left[1+24 s^{-2} \exp \left(8 x_{r}\right)\right] \\
& \times\left[a \exp \left(b p_{t}^{2}\right) \exp \left(-c x_{r}\right)\right] .
\end{aligned}
$$

Here $p_{t}$ and $E$ are the transverse momentum and center-of-mass kinetic energy of antiprotons produced in collisions of protons travelling along the $z$ axis with target nuclei characterized by empirical constants $a, b$, and $c$ and by total proton absorption cross section $\sigma_{a b s}$; $s$ is the square of the center-of-mass energy and $x_{r}$ $=E / E_{\max }$, where $E_{\max }$ is the maximum kinematically allowed antiproton center-of-mass kinetic energy. For hydrogen, $a=1, b=c=0$, and the last factor becomes unity, but the measurement-derived values given by Hojvat and van Ginneken for common target materials are all of order one (Table I). Fermi motion effects are not included in the formula, but values can, with care, be interpolated for iridium and other common metal targets.

The primary proton beam is focused in both planes by optical elements to form an image inside the target cylinder that matches its size and shape as far as possible. Evidently the primary-beam emittance will limit the overall emittance of the secondary (antiproton) beam, usually required to be as small as possible. In the ideal case, the target should be long enough relative to the collision length $L_{\text {coll }}$ to make sure that the protons interact, but short compared with the absorption length $L_{\text {abs }}$ for antiprotons, so that most of the antiprotons will leave the cylinder. As $L_{\text {coll }} \approx L_{\text {abs }}=50 \mathrm{~mm}$ for iridium, the aim will normally be to produce a beam waist within a 50-mm-long cylinder. The emerging antiprotons must then be collected by another double-focusing element. Of the various possibilities, magnetic horns are usually preferred when robustness, ease of repair, and simplicity are the paramount considerations. When this is not so, lithium lenses and active (or current-carrying) targets may be used, resulting in yields that are $20-40 \%$ higher.

The usable antiproton intensity is limited by several factors, including the acceptable thermal shock to the target produced by the proton beam. For the maximum allowable target temperature (about $1500{ }^{\circ} \mathrm{C}$ ), the pro-

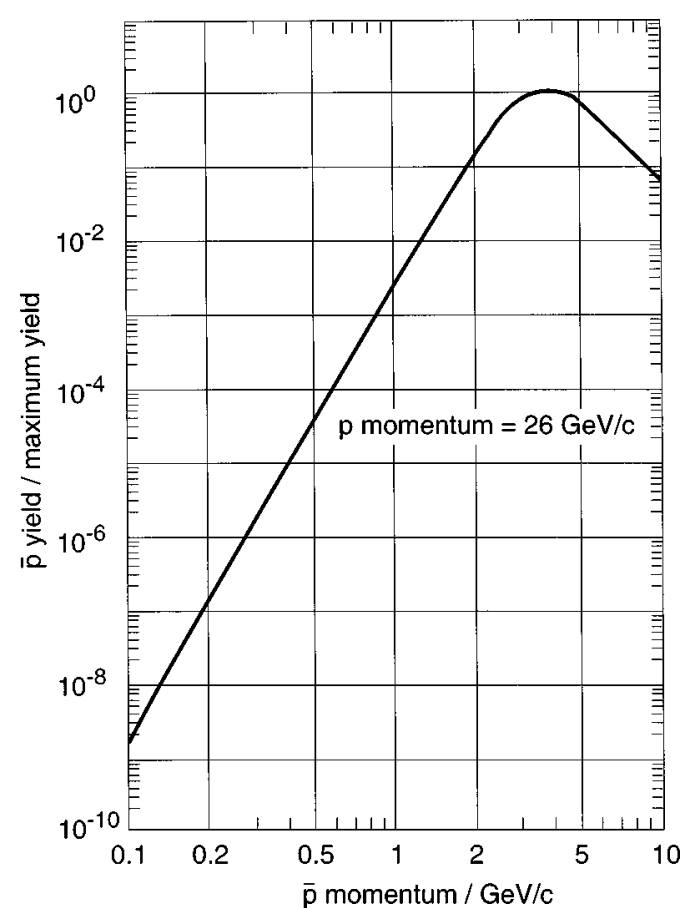

FIG. 1. Normalized antiproton yield (antiprotons per proton) at $26 \mathrm{GeV} / c$ proton-beam momentum. The normalization is chosen so that the yield is one at the maximum.

ton pulse intensity delivered to a $1-\mathrm{mm}^{2}$ area must be less than $\sim 6 \times 10^{14}$ per GeV/c. This is $2.5 \times 10^{13}$ protons for $26 \mathrm{GeV} / c$ (the CERN case), at which momentum Möhl (1997) calculates the yield as a function of antiproton collection momentum as shown in Fig. 1. The yields are normalized to their value at the $3.5-\mathrm{GeV} / \mathrm{c}$ maximum, and show a very steep falloff on either side of it, so it is important not to stray far from this value. The dependence of the antiproton momentum for maximum antiproton yield on the incident-beam momentum is shown in Fig. 2.

Some of the characteristics of the CERN antiproton source are displayed in Table II and compared with those at Fermilab, where the proton-beam energy is $120 \mathrm{GeV} / c$ and the momentum for maximum yield is then $\sim 9 \mathrm{GeV} / c$. Although the production cross section is 20 times larger at the Fermilab proton-beam energy, the yield (antiprotons per proton) is only four times

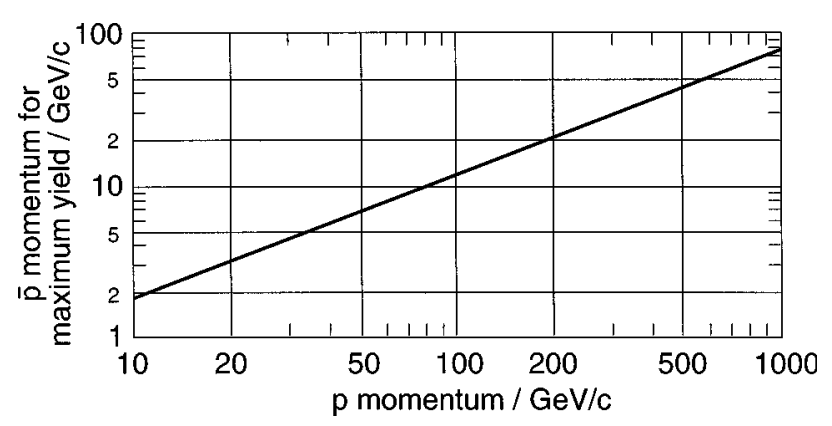

FIG. 2. Antiproton momentum for maximum yield vs incidentproton momentum. 
TABLE II. Comparison of CERN and Fermilab antiproton sources: for Fermilab the upgrading program quoted in Church and Marriner (1993) has been anticipated; for CERN the measured yield with magnetic horn has been used.

\begin{tabular}{lll}
\hline \hline Machine & $\begin{array}{c}\text { CERN } \\
\text { Antiproton Collector }\end{array}$ & $\begin{array}{c}\text { Fermilab } \\
\text { debuncher }\end{array}$ \\
\hline Production momentum $(\mathrm{GeV} / c)$ & 26 & 120 \\
Collection momentum $(\mathrm{GeV} / c)$ & 3.5 & 9 \\
$\bar{p} / \mathrm{sr} / \mathrm{GeV} / \mathrm{c} /$ Interacting $p$ & 0.013 & 0.25 \\
Acceptances $\quad A_{h}(\pi \mathrm{mm} \mathrm{mrad})$ & 200 & 25 \\
$A_{v}(\pi \mathrm{mm} \mathrm{mrad})$ & 200 & 25 \\
$\sqrt{A_{h} A_{v}} \times \Delta p / p\left(\pi \mathrm{mm} \mathrm{mrad} \times 10^{-3}\right)$ & 60 & 40 \\
Yield $(\bar{p} / p)$ & $12 \times 10^{3}$ & $10^{3}$ \\
Protons per pulse & $3.5 \times 10^{-6}$ & $14 \times 10^{-6}$ \\
Antiprotons per pulse & $1.5 \times 10^{13}$ & $0.5 \times 10^{13}$ \\
\hline
\end{tabular}

higher than at CERN, largely due to the target-heating limitation mentioned above.

Our brief tour of the world's antiproton facilities terminates in the company of Peaslee (1996) and Seth (1997), who describe the current situation at the Brookhaven Alternating-Gradient Synchrotron (AGS) together with a plan for the development of an antiproton facility based on the AGS upgrade to serve as injector for the Relativistic Heavy-Ion Collider (RHIC).

\section{B. Accumulating antiprotons}

From the early days, the difficulties of working with antiprotons were compounded by the large numbers of pions and other negatively charged particles that were inevitably produced in the target along with the antiprotons. Until the early 1970s all $\bar{p}$ beams were therefore "unseparated," which is to say that they were usually $\pi^{-}$beams in which the antiprotons constituted a small background. Electrostatic separators (de Raad, Minten, and Keil, 1966), which work by deflecting particles of equal momentum but different mass by different amounts when they pass between the plates of a condenser, certainly improved the background problem, but what was really needed was a technique for collecting antiprotons produced near the momentum of maximum yield over an extended period, without also collecting particles of other types.

This need was eventually filled by the advent of the beam cooling techniques that culminated in the discovery of the $W$ and $Z$ bosons at the CERN $300-\mathrm{GeV}$ proton-antiproton collider $(S p \bar{p} S)$ in 1983. The array of machines assembled for this purpose (Fig. 3) at CERN again exemplifies most of the features of a typical modern antiproton "factory." Particles emerging at $3.5 \mathrm{GeV} / c$ from a target station, as described above, entered a storage ring known as the antiproton accumulator, or AA (Evans et al., 1989), where unwanted charged particles (mainly pions and electrons) rapidly decayed or radiated energy via synchrotron radiation until they collided with the beam pipe. The horizontal and vertical
AA acceptances $\left(E_{H}\right.$ and $\left.E_{V}\right)$ were both of order 100 $\times \pi \mathrm{mm} \times \mathrm{mrad}$, and its momentum acceptance $\Delta p / p$ was $0.7 \%$. The single-pulse yield of about $10^{7}$ antiprotons from the target station described above occupied about $50 \%$ of this phase-space volume, while the $W$ and $Z$ experiments required building up an AA stack containing several times $10^{11}$ particles (Evans et al., 1989). This enormous demand could only be satisfied if some way could be found to compress the phase-space density of single pulses by a factor of $10^{8}$, thereby making room for the accumulation of many successive pulses prior to acceleration to $300 \mathrm{GeV}$.

Liouville's theorem normally forbids any such reduction of phase-space volume for a fixed-momentum beam. Stochastic cooling (Möhl et al., 1980) avoids this constraint by the application of repeated corrections to the particles' orbits in the storage ring by means of sig-

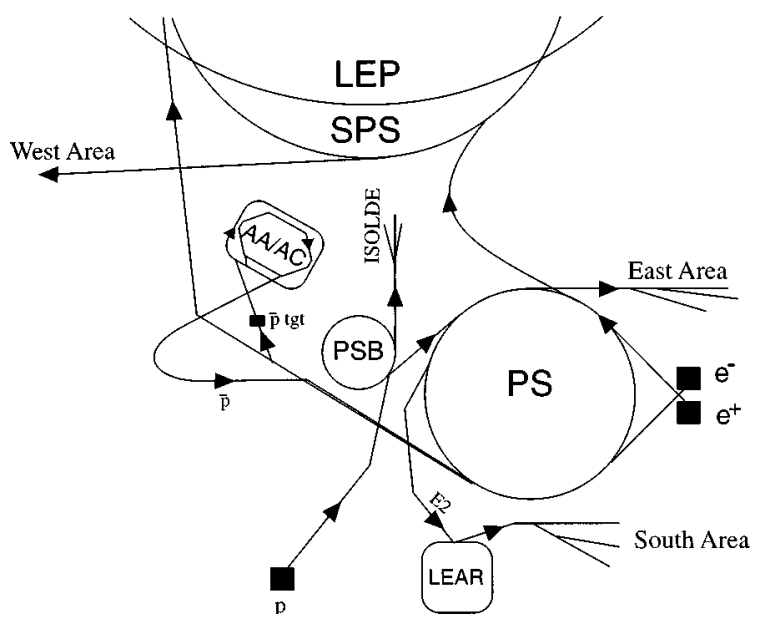

FIG. 3. The CERN antiproton factory (not to scale) showing also the $e^{+}$and $e^{-}$sources, the Large Electron-Positron collider (LEP), and the Super Proton Synchrotron (SPS) formerly used to study $300-\mathrm{GeV} p-\bar{p}$ collisions. The Antiproton Collector (AC) ring is the outer of the two concentric storage rings labeled AA/AC. PSB is the Proton Synchrotron (PS) booster ring. 


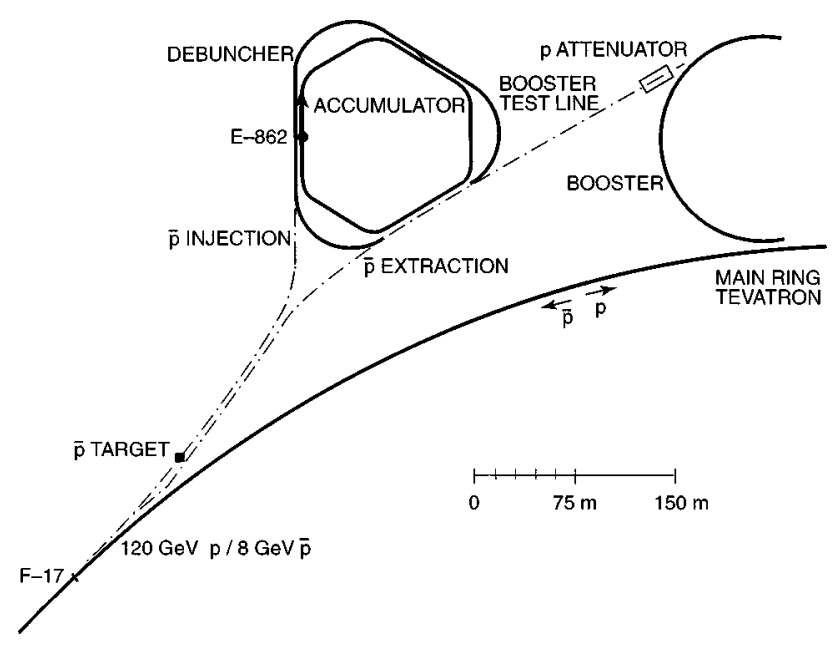

FIG. 4. The FNAL antiproton factory. The Debuncher and Accumulator correspond roughly to the CERN AA and AC. The $\bar{H}$ experiment (see Fig. 18) described in Sec. VI.D occupies the position labeled E-862.

nals from pickup electrodes that sense their mean deviation from the ideal orbit. ${ }^{9}$

The final element required for the $W$ and $Z$ experiments was the Antiproton Collector (AC), a supplementary storage ring concentric to the AA; this was added in 1984 (AC Design Study Team, 1983). The longitudinal emittance of a bunched antiproton beam of duration $\Delta t$ and energy spread $\Delta E$ (set by the target length and the beam energy) is $\pi \times \Delta E \times \Delta t$. The $\Delta t$ of the antiprotons is of course equal to that of the protons; consequently the shorter the duration of the proton pulse, the smaller will be the energy spread after debunching in the storage ring and the more easily can the antiprotons then be cooled and stacked (Möhl, 1997). The intermediate AC ring performed these debunching and precooling operations separately prior to the transfer of the antiprotons to the AA. This separation of functions resulted in a factor-of-5 increase in the the AA capacity.

The Fermilab Debuncher/Accumulator (Fig. 4) corresponds roughly to the CERN AC/AA, but is physically considerably larger (some $474 \mathrm{~m}$ in circumference, as compared to $182 \mathrm{~m}$ ). For deceleration to low energies the higher storage momentum is a disadvantage, and the high-energy proton-antiproton Tevatron collider remains as the prime user of the Fermilab source long after the closure of the CERN collider.

\section{DECELERATING GeV ANTIPROTONS}

In Sec. II we discussed the ways in which the specific needs of high-energy experiments with antiprotons have been filled since 1955, the requirements of experiments with antiprotons at extremely low energies having al-

\footnotetext{
${ }^{9}$ What is cooled is the transverse or longitudinal energy of individual antiprotons relative to the mean motion of the whole pulse.
}

ways been rather incidental to these endeavors. We now turn to the unusual problems posed by the latter experiments. The first problem is that electromagnetic traps are limited by practical considerations to depths of a few tens of $\mathrm{kV}$; antiproton confinement in such devices will therefore mean reducing their energy from the typical production values of order $\mathrm{GeV}$ to some tens of $\mathrm{keV}$. For confinement in exotic atoms, the corresponding figure is some tens of $\mathrm{eV}$ (this being the energy at which the capture cross section becomes dominant).

\section{A. From $\mathrm{GeV}$ to $\mathrm{MeV}$}

It should not, therefore, be surprising that solutions to the puzzle of how to obtain low-energy antiprotons from high-energy ones were a long time coming and that study of their intrinsic properties under ideal conditions was for many years out of the question. The Liouville condition that the phase-space volume occupied by a collection of particles remains constant is true only at constant momentum; if the particles undergo deceleration, the theorem ensures that this volume will increase with the inverse third power of the momentum (Möhl, 1997).

As with the antiproton collection/storage problem described in Sec. II, the solution to this new Liouvilletheorem problem came from phase-space cooling. It was quickly realized that the rapid phase-space blowup that inevitably would accompany deceleration below the 3.5- GeV/c AA/AC storage momentum could be compensated for by cooling the beam pulse at one or more intermediate momenta.

\section{LEAR}

Undoubtedly the most important dividend of the CERN 300-GeV $p \bar{p}$ collider for low-energy antiproton studies was the adaptation of AA cooling techniques to a new storage ring known as LEAR (Low-Energy Antiproton Ring); indeed this machine (LEAR Design Study Team, 1980) survived the $S p \bar{p} S$ itself for many years. The sequence of events in use for decelerating antiprotons began with the return from the AA to the proton synchrotron, approximately once every 30 minutes, of bunches containing a few $\times 10^{9}$ particles and extracted from the $3.5-\mathrm{GeV} / \mathrm{c} 10^{12}$-strong AA stack. In the proton synchrotron they were decelerated to $0.6 \mathrm{GeV} / c$ before transfer to the LEAR ring proper (Fig. 5), where cooling was applied after successive deceleration to three or four intermediate momenta. At and below $300 \mathrm{MeV} / c$, the electron cooling technique, as initially demonstrated by Budker et al. (Budker, 1966; Budker et al., 1975; Budker and Skrinsky, 1978) was found to be more efficient than stochastic cooling and permitted energies as low as $2 \mathrm{MeV}$ (momentum $\sim 61 \mathrm{MeV} / c$ ) to be attained. In electron cooling the antiprotons transferred energy by collisions to a velocity-matched (comoving) electron beam; the increase in their phase-space volume was effectively transferred to the phase space of the electron beam. This, being continuously replenished with cold electrons from its source, carried off the antiprotons' 


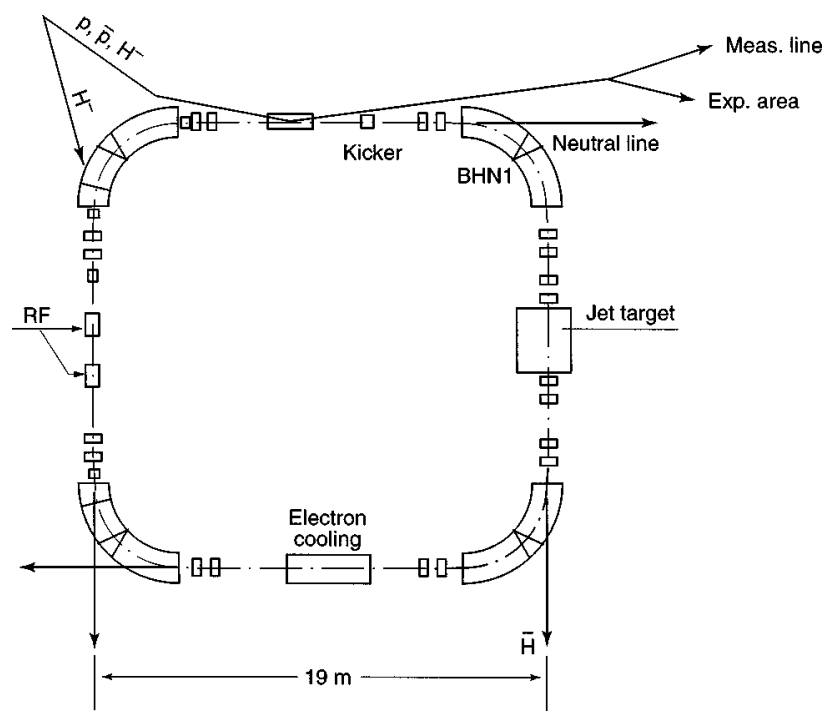

FIG. 5. The CERN Low-Energy Antiproton Ring, LEAR. For most experiments, the antiproton beams were ejected into an experimental hall at the right. For the $\bar{H}$ experiment described in Sec. VI.D, the internal circulating beam was used with a xenon gas jet target in the indicated position.

surplus relative energy into the electron-beam dump. A final application of cooling at the extraction momentum compressed the beam phase space to the emittance required in the external beamlines. Typical $106 \mathrm{MeV} / c$ $(6.0 \mathrm{MeV})$ beamline values were $1 \pi \mathrm{mm} \times \mathrm{mrad}$ and $\Delta p / p=5 \times 10^{-4}$. In addition to these deceleration capabilities, LEAR could also re-accelerate the $600-\mathrm{MeV} / c$ antiprotons to $1.6 \mathrm{GeV} / c$ for special purposes, such as the antihydrogen production experiment discussed in Sec. VI.D.

\section{The Antiproton Decelerator, $A D$}

LEAR was formally closed in December 1996, after fourteen years of service which saw the achievement of many of the experimental results cited in Secs. IV and VI below. It will by the end of 1998 be replaced for these purposes by the Antiproton Decelerator (AD). The AD (Berlin et al., 1996; Baird et al., 1997; Maury, 1997), being specifically directed towards the extremely low energies required for the kind of experiments described below, will retain most of the features of LEAR without being burdened by the high-luminosity requirements for microbarn or nanobarn-scale meson spectroscopy experiments, although its antiproton flux will be lower than that of LEAR by a factor of about 10 . Strictly speaking, it is not a new machine but a modification of an old one, the Antiproton Collector (AC), referred to in Sec. II (Fig. 6). The main production features of the antiproton target station described above will be maintained, together with some advantages derived from developments related to the CERN Large Hadron Collider. Once in the $\mathrm{AD}$ at $3.5 \mathrm{GeV} / c$, antiprotons will be stochastically cooled to $5 \pi \mathrm{mm} \times \mathrm{mrad}$ in transverse planes and $\Delta p / p=10^{-3}$, then decelerated to $2 \mathrm{GeV} / c$ and stochastically cooled again. Electron cooling will be used after subsequent deceleration steps to the $300-\mathrm{MeV} / \mathrm{c}$ and $100-\mathrm{MeV} / \mathrm{c}$ plateaus, using hardware transferred from LEAR. Table III summarizes the expected emittances, momentum spreads, and cooling times at the various intermediate momenta.

The new AD experimental area will occupy the space inside the AC ring (Fig. 6), the elements of the AA being removed for this purpose. Ejection to this area will not differ substantially from the system formerly used to transfer particles from the AC to the AA. After

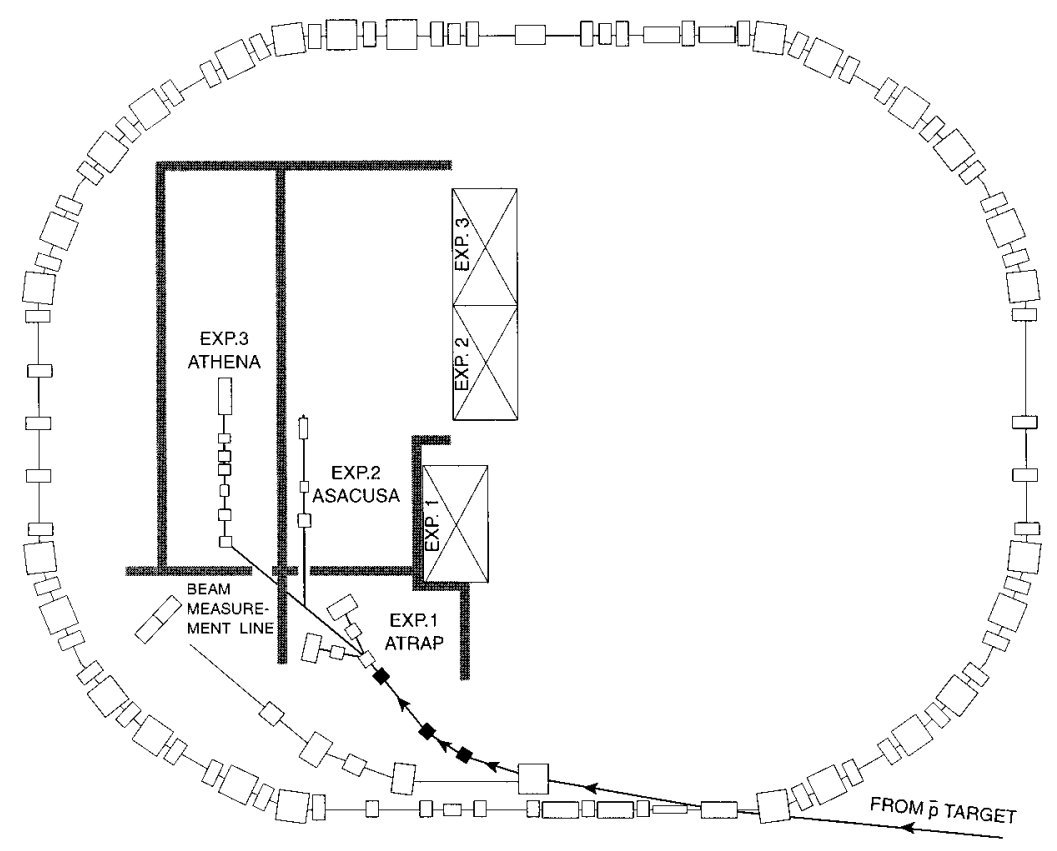

FIG. 6. The CERN Antiproton Decelerator (AD), in large measure a refurbished version of the Antiproton Collector (AC) of Fig. 3. 
TABLE III. Transverse emittances $\left(\epsilon=2 \sigma^{2} / \beta\right)$ in the Antiproton Decelerator (AD) ( $\epsilon_{i}$ and $\left.\epsilon_{f}\right)$ and momentum spreads $\left(\Delta p / p_{i}\right.$ and $\left.\Delta p / p_{f}\right)\left(\Delta p=4 \times \sigma_{p}\right)$ before and after cooling at intermediate momenta $p$, and cooling times $t$. Only adiabatic increase due to deceleration is considered.

\begin{tabular}{ccccccc}
\hline \hline $\begin{array}{c}p \\
\mathrm{GeV} / c\end{array}$ & \multicolumn{1}{c}{$\epsilon_{i}$} & $\begin{array}{c}\epsilon_{f} \\
\pi \mathrm{mm} \times \mathrm{mrad}\end{array}$ & $\Delta p / p_{i}$ & $\Delta p / p_{f}$ & $\begin{array}{c}t \\
\mathrm{~s}\end{array}$ & Cooling process \\
\hline 3.5 & 200 & 5 & 1.5 & 0.1 & 20 & Stochastic \\
2.0 & 9 & 5 & 0.18 & 0.03 & 15 & Stochastic \\
0.3 & 33 & 2 & 0.2 & 0.1 & 6 & Electron \\
0.1 & 6 & 1 & 0.3 & 0.01 & 1 & Electron \\
0.1 (bunched) & - & 1 & - & 0.1 & - & Electron \\
\hline \hline
\end{tabular}

electron cooling at $100 \mathrm{MeV} / c$, it should be possible to deliver standard operation pulses of about $10^{7}$ antiprotons to the experiments in a pulse of $0.2-0.5 \mu s$ duration. This bunched operation to the experimental hall is all that is required for the experiments on antihydrogen and antiprotonic helium which will form the backbone of the AD experimental program (Sec. VIII). The cycle time between particle transfers from the proton synchrotron is expected to be about one minute when sufficient time has been allowed for the cooling at intermediate momenta. A further option is the possibility of stacking two to ten $3.5-\mathrm{GeV} / c$ proton synchrotron pulses in the $\mathrm{AD}$, increasing the intensity per cycle by an order of magnitude.

\section{B. Deceleration below $1 \mathrm{MeV}$}

A practical lower limit for the standard deceleration techniques previously used in LEAR, and now to be used in the $\mathrm{AD}$, is approximately $5 \mathrm{MeV}(100 \mathrm{MeV} / c)$. Although several methods are available for reaching the practical upper limit of electromagnetic trap structures $(\sim 50-100 \mathrm{keV})$, they are often less than ideal for all purposes, and the choice among them is usually made according to the way the antiprotons will be used at the lower energy.

\section{Passive energy dissipation}

By passive degradation we mean slowing of particle beams by passage through thin foils and/or gases, no attempt being made to compensate for or circumvent the inevitable effects of multiple scattering and energy spread.

In the sense that the beam particles lose energy to the electrons of the degrader material, these are also Liouville-theorem effects, diverting beam particles to phase-space regions lying outside the acceptance of experimental apparatus downstream of the degrader so that they become unusable.

Passive degradation is therefore often characterized by high particle loss rates, sometimes reaching $99 \%$ or more. Thus when a thin foil serves the dual purpose of energy degrader and window into a Penning trap (Gabrielse et al., 1989; Holzscheiter et al., 1996), most particles either annihilate within the foil or leave it with kinetic energy greater than the trap depth. The reason why such disadvantages do not disqualify passive degradation from any interest at all is that many studies of very-low-energy antiprotons require few particles anyway.

In other experiments, thin foils have been used as windows into high-density gas targets, as discussed by Nakamura et al. (1994) and Torii et al. (1997). These authors describe situations in which the exit energy from the foils is high enough for the annihilation losses within them to be negligible, but the residual range of antiprotons in the gas is short, and the stopping volume correspondingly small.

In both types of experiment, the need for beam diagnostic devices complicates the simple geometry in which the beam pipe is closed by a window that leads directly into the trap volume or gas target. Thus the particles must normally traverse a beam-pipe sealing foil into a sequence of air drift spaces, scintillation counters, and/or tracking chambers before traversing the final window into the experimental apparatus. Sometimes a variablepressure gas cell is also added to allow fine tuning of ranges and energies.

The energy and angular distributions of antiprotons emerging from the window into the experimental apparatus must then be simulated by Monte Carlo calculations in which the geometrical layout is fully modelled. In addition to specifying the thickness and position of foils, intermediate air paths, etc., such calculations must take into account several deviations from the BetheBloch $d E / d x$ formula for projectile velocities $\beta=v / c$ below about $Z \alpha c$. In this domain the implicit assumption that the scattering material's electrons are free, as well as various other assumptions and approximations used in deriving the formula, are no longer justified (for a discussion see, e.g., ICRU Report 49, 1993; LodiRizzini and Zenoni, 1997). Furthermore the loss of validity of the impulse approximation (according to which the projectile does not deviate from a straight line while passing through the atom) introduces important processes at low energies that depend on the sign of its charge.

At $\bar{p}$ kinetic energies above $1 \mathrm{MeV}$ the Bethe-Bloch formula is the standard stopping-power formula for a projectile of charge ze traversing a degrading foil (or 
TABLE IV. Calculated energy losses $\delta T$ of antiprotons in the windows and counters used by Iwasaki et al. (1991) to deliver antiprotons into a target chamber for subsequent stopping in gas. $x$ is the distance from the beamline window in beam direction, $d$ the thickness of the material, $d^{\prime}$ the surface density of the material in $\mathrm{mg} / \mathrm{cm}^{2}$, and $T$ the kinetic energy of the $\bar{p} s$ after they traverse the material.

\begin{tabular}{lllcrcc}
\hline \hline & \multicolumn{1}{c}{$\begin{array}{c}x \\
\text { Material }\end{array}$} & $\mathrm{cm}$ & $d$ & $\begin{array}{c}d^{\prime} \\
\mathrm{mg} / \mathrm{cm}^{2}\end{array}$ & $\begin{array}{c}\delta T \\
\mathrm{MeV}\end{array}$ & $\begin{array}{c}T \\
\mathrm{MeV}\end{array}$ \\
\hline Beam Energy & & & & & & 5.85 \\
Beam Window & Be & 0 & $100 \mu \mathrm{m}$ & 18.5 & 1.15 & 4.70 \\
Gap & Air & & $3.5 \mathrm{~cm}$ & 4.2 & 0.30 & 4.40 \\
B counter & Pl. Scint. & 3.5 & $100 \mu \mathrm{m}$ & 10.3 & 0.97 & 3.43 \\
Gap & Air & & $2 \mathrm{~cm}$ & 2.4 & 0.22 & 3.21 \\
Degrader & Kapton & 5.5 & $50 \mu \mathrm{m}$ & 7.0 & 0.77 & 2.44 \\
Gap & Air & & $2 \mathrm{~cm}$ & 2.4 & 0.28 & 2.16 \\
Target Window & Kapton & 7.5 & $50 \mu \mathrm{m}$ & 3.5 & 0.50 & 1.66 \\
\hline \hline
\end{tabular}

target) of atomic charge and number $(Z e, A)$ and mean ionization potential $I$ (Particle Data Group, 1996):

$$
-\frac{d E}{d x}=4 \pi r_{e}^{2} m_{e} c^{2} N_{A} \frac{Z}{A} \frac{1}{\beta^{2}} z^{2} L,
$$

where $r_{e}$ is the classical electron radius and $N_{A}$ Avogadro's number.

The value of the $4 \pi r_{e}^{2} m_{e} c^{2} N_{A}$ term is $0.307 \mathrm{MeV} \mathrm{cm} / \mathrm{Mol}$, and the factor $L$ has the slowly varying $\beta$ dependence

$$
L=\frac{1}{2} \ln \left(\frac{2 m_{e} c^{2} \gamma^{2} \beta^{2} T_{\max }}{I^{2}}-\beta^{2}-\frac{\delta}{2}\right) .
$$

Here $T_{\max }$ is the maximum possible energy transfer to an atomic electron $\left(\sim 2 m_{e} c^{2} \beta^{2} \gamma^{2}\right.$ at all energies of interest here), and $\delta / 2$ is a density correction, negligible for particles with $v \ll c$.

The above-mentioned deviations from Eq. (2) are usually parametrized by replacing $L$ with a $z$-dependent polynomial series:

$$
L \rightarrow L_{0}+z L_{1}+z^{2} L_{2}+\cdots .
$$

Thus $L_{0}$ corresponds to $L, L_{1}$ gives the $z^{3}$ (i.e., signdependent) polarization term usually known as the Barkas term (Barkas et al., 1956), and $L_{2}$ gives the (signindependent) $z^{4}$ (Bloch) term.

In this low-energy region, computer simulations usually involve a mixture of empirical data and energy-loss formulas. A wealth of such data is available for the stopping power $S \equiv d E / d x$ of protons down to very low energies. Using a parametrization proposed by Varelas and Biersack (1970), Andersen and Ziegler (1977) developed a table of stopping-power parameters for all elements, which took all measurements until 1977 into account. This parameter list has been constantly updated (Ziegler, 1991) and is claimed to be valid down to $1 \mathrm{keV}$.

For antiprotons, major Barkas-effect corrections (Barkas et al., 1956) are necessary in the energy region below $1 \mathrm{MeV}$. They reach about $30 \%$ of the proton stopping-power values at the energy-loss maximum (the so-called Bragg peak). Data on these corrections for heavy negatively charged particles $\left(\mu^{-}, \pi^{-}, \bar{p}\right)$, however, are rather scarce. For antiprotons, measurements have been performed only for hydrogen (Adamo et al., 1993) and several metals (Mbller et al., 1997). The Barkas term $L_{1}$ is rather well reproduced by calculations based on the harmonic-oscillator model (Mikkelsen, 1992) down to about $100 \mathrm{keV}$. Nevertheless some uncertainty remains for all low-energy-range calculations performed for antiprotons. For the moderation of 5.3-MeV $(100 \mathrm{MeV} / c)$ antiprotons to several tens of $\mathrm{keV}$ these uncertainties play a minor role: The particle range $R$ increases with kinetic energy $T$ roughly as $T^{1.7}$, and the 5.3-MeV antiprotons traverse about $94 \%$ of their range at energies above $1 \mathrm{MeV}$, where the Barkas effect is negligible. This effect grows to the aforementioned $30 \%$ level between $1 \mathrm{MeV}$ and $\approx 100 \mathrm{keV}$. As the uncertainties of the theoretical energy-loss formulas are also less than $30 \%$, path-length calculations in this energy region should be accurate at the percent level.

A typical example of accurate modeling of the degrading process is that by Iwasaki (1994; see also Nakamura et al., 1994). These authors used both the semiempirical Andersen and Ziegler $d E / d x$ parametrization (1977) and Barkas-effect corrections in a calculation for a 5.6-MeV antiproton beam entering a high-density helium chamber through various degrader elements. Their calculated energy losses are summarized in Table IV; the calculated residual ranges in the helium target are in good agreement with the values finally measured via the distribution of annihilations at rest (Table V). ${ }^{10}$

When similar degrader geometries are used to load antiprotons into Penning traps (Sec. IV.A.1 below), the foil exit energy must be chosen so as to maximize the number of antiprotons emerging into a given energy range, the trap "depth," of order $\mathrm{keV}$ or tens of $\mathrm{keV}$ as we saw above. Under these conditions the total degrader thickness must be of the same order as the mean range $R_{0}$, and many antiprotons will annihilate in it. Nevertheless, measured efficiencies for $\mathrm{keV}$ (Gabrielse

\footnotetext{
${ }^{10} \mathrm{We}$ defer to Sec. IV.B.1 a more detailed discussion of degradation processes in the extremely-low-energy region at which antiproton capture into exotic atoms occurs.
} 
TABLE V. Calculated and measured values for range and range straggling in helium gas of different pressure.

\begin{tabular}{lcccc}
\hline \hline Pressure & \multicolumn{2}{c}{$3 \mathrm{~atm}$} & \multicolumn{2}{c}{$10 \mathrm{~atm}$} \\
\hline & $\langle R\rangle$ & $\delta R$ & $\langle R\rangle$ & $\delta R$ \\
& $\mathrm{~cm}$ & FWHM, cm & $\mathrm{cm}$ & FWHM, cm \\
Standard Calc. & $9.0 \pm 2$ & 3.8 & $2.7 \pm 1.5$ & 1.2 \\
Incl. Barkas & $11.9 \pm 2$ & 3.8 & $3.6 \pm 1.5$ & 1.2 \\
Experiment & $13.5 \pm 1$ & $3 \pm 1$ & $6.6 \pm 1$ & $\approx 1$ \\
\hline \hline
\end{tabular}

et al., 1989) and tens of $\mathrm{keV}$ (Holzscheiter et al., 1996) samples are now well accounted for by modeling programs.

Multiple scattering and energy spread, two effects already addressed briefly, limit the efficiency of loading antiprotons into traps considerably. During slowing down to the Bragg maximum, the energy distribution is drastically widened. It was shown by Fano (1953), Payne (1969), and Daniel (1977) that the width of the energy distribution of an ensemble of particles varies with the stopping power, just by the difference in stopping power between the fastest and the slowest particles. This effect is even more important than the effect of range straggling due to the statistical nature of the interaction process. This means, for example, that an antiproton beam of $100 \mathrm{MeV} / c(5.3 \mathrm{MeV})$ and LEAR quality $(\Delta T / T$ $\sim 10^{-3}$ ) is widened during its way through a silicon moderator (here reliable stopping-power data are available for $T<1 \mathrm{MeV}$ ) to $\Delta E=27 \mathrm{keV}$ at $100 \mathrm{keV}$, which gives a $\Delta T / T$ of $27 \%$. At the rather low acceptance of traps this is a real drawback. It may be overcome, however, by the use of RFQ (radio-frequency quadrupole) deceleration, as discussed in Sec. III.B.3.

Multiple scattering (Molière, 1947; Marion and Zimmerman, 1967) increases the lateral size of the $\bar{p}$ beam and therefore decreases its particle density. Slowing down a $\bar{p}$ beam from $100 \mathrm{MeV} / c(5.3 \mathrm{MeV})$ to $100 \mathrm{keV}$ with a $\mathrm{Be}$ moderator results in a mean divergence of about $10^{\circ}$. To avoid large radial dimensions, which make the trapping efficiency low, the distances in the experimental setup have to be kept short and low- $Z$ moderators (like beryllium or helium) are mandatory.

\section{The cyclotron trap, or "anticyclotron"}

A variant on passive dissipation, the so-called cyclotron trap (Simons, 1993) developed for studies of exotic atoms, has in addition provided many data on the stopping power $(S)$ of gases in this energy region. This device is a cyclotron operating in inverse (i.e., decelerating) mode. However, the deceleration is not produced by a radio-frequency electric field, but by dissipative forces in a low-pressure gas introduced into what would, in a normal cyclotron, be the vacuum chamber. Antiprotons or other particles enter the gas through a beryllium window placed in the $z-\theta$ plane near the outer edge of this chamber. The field is of a conventional cyclotron type, with $z$-component $B_{z}$ up to about $1.6 \mathrm{~T}$ and the usual radial and axial inhomogeneities necessary to ensure orbital cyclotron stability via betatron oscillations.

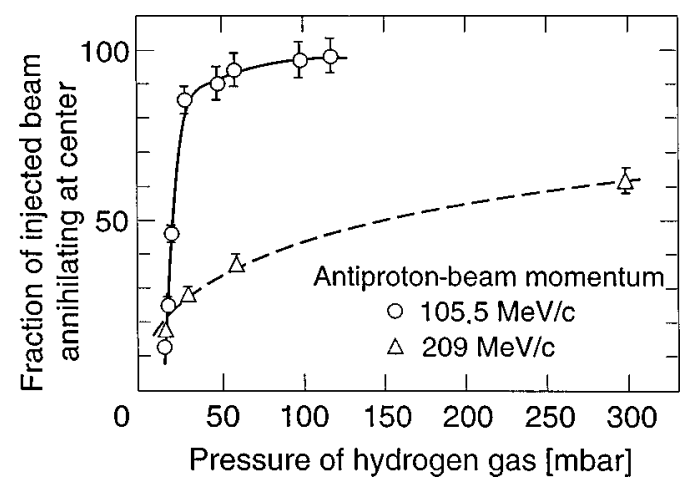

FIG. 7. Number of antiprotons annihilating at the center of the cyclotron trap vs degrader gas pressure, as a fraction of the number entering the trap, at two values of the beam momentum.

In the absence of dissipative forces (i.e., in vacuo or with extremely low-pressure gas) the beam would return to the window and annihilate there after a few orbits. If, on the other hand, the gas pressure is so high that too much energy is dissipated per turn, the orbital stability will be destroyed. An intermediate range of pressures exists, however, within which the dissipation per turn is sufficiently adiabatic to maintain the radial and axial stability, but high enough to allow a useful fraction of the injected antiprotons to spiral down towards the center of the magnet in stable orbits. The foil thickness, and its azimuthal and radial positions, must be carefully tuned for each gas pressure and beam momentum with the help of computer simulations to ensure that the beam leaves the foil with mean radial momentum close to zero and azimuthal momentum slightly smaller than the corresponding stable-orbit radius.

Typically, the exit energy is in the region of one $\mathrm{MeV}$, and a large fraction of the particles is accepted into stable orbits at hundreds of mbar gas pressure. As the pressure is reduced below a few tens of mbar, this fraction becomes very small owing to insufficient dissipation during the first few turns. Figure 7 demonstrates this effect as measured by the fraction of antiprotons that annihilates at the center of the magnet after slowing down to the eV-scale energy at which exotic atoms are formed in the dissipating gas. This, the normal fate of the antiprotons in this device, will be described in Secs. IV.B.1 and IV.B.2, but attempts have been made (Sec. IV.A.1) to extract them from the magnet center through an axial borehole. Operated in this mode, with injection at high energy and extraction at low energy, the cyclotron trap has usually been referred to as an "anticyclotron."

\section{Radio-frequency quadrupoles}

A third technique for decelerating antiprotons below a few $\mathrm{MeV}$ kinetic energy is that of the radio-frequency quadrupole (RFQ) proposed by Kapchinsky and Teplyakov (1970), but used in decelerating, rather than the usual accelerating mode. 


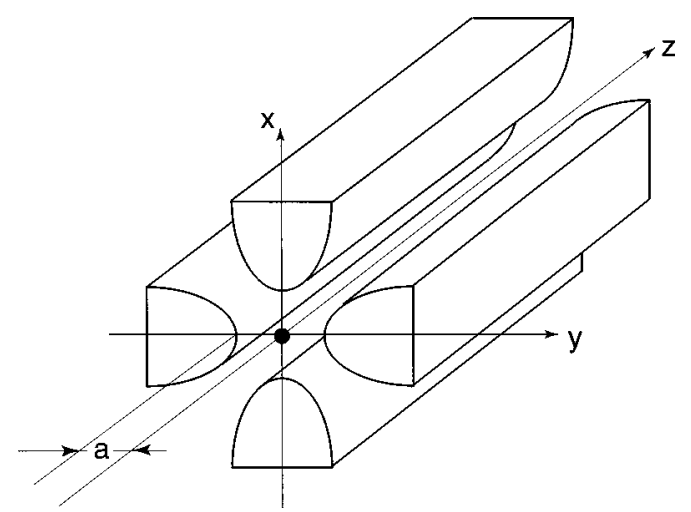

FIG. 8. Principles of a Radio-Frequency Quadrupole (RFQ). A voltage $+\left(V_{0} / 2\right) \sin \omega t$ is applied to the two electrodes on the $x$ axis, a voltage $\left(-V_{0} / 2\right) \sin \omega t$ to the electrodes on the $y$ axis.

Electric quadrupole fields, unlike their magnetic counterparts, develop deflecting forces that do not depend on the particle velocity and are therefore effective for particles down to zero kinetic energy. Thus a dc voltage applied to four hyperbolic-section electrodes (known as vanes) arranged as in Fig. 8 would produce central restoring forces (i.e., linearly increasing with increasing distance from the symmetry axis) on particles moving at any speed in the $z$ direction. As with the magnetic quadrupole case, focusing in one plane implies defocusing in the other, and a simple transport line relies on the principle of "alternate-gradient" focusing, in which a spatial sequence of short (constant-voltage) quadrupoles with alternating polarity ensures restoring forces in both planes. Another solution, due to W. Paul, to the problem of double-focusing (see Bruck, 1966) is to excite a long quadrupole by an rf voltage; a beam traveling along $z$ will then experience focusing and defocusing fields in turn just as if it were traversing a series of short quadrupoles.

Simple hyperbolic vanes as in Fig. 8 cannot of course produce the $z$ fields necessary to accelerate the particles. However, if opposite pairs are machined in such a way that the distance $(2 \times a)$ between them varies cyclically along $z$, a weak axial-field component results (Schempp, 1992; Weiss, 1995) with the standing-wave form

$$
E_{z}=\frac{V}{2} k A_{10} I_{0}(k r) \sin (k z) \sin (\omega t+\phi) .
$$

Here $I_{0}$ is the Bessel function of zeroth order, and $A_{10} \equiv\left(m^{2}-1\right) /\left[m^{2} I_{0}(k a)+I_{0}(m k a)\right]$ is a dimensionless geometrical function (the acceleration parameter) of the minimum $(a)$ and maximum $(m \times a)$ distances of the vane surface from the $z$ axis; $k=2 \pi / \beta \lambda$ where $\beta \times c$ is the particle velocity and $\lambda$ is the free-space rf wavelength, $\omega$ is the rf angular frequency, and $\phi$ is the phase angle between the field and the particle when it enters the RFQ.

The depth $(m-1) a$, and pitch $\beta \lambda$ of the vane modulations can be chosen such that the $z$ field remains phase synchronized with the particles as their velocity increases under its action. The axial perturbation there- fore accelerates, its weakness being compensated for by the fact that it acts over the whole length of the cavity (typically several meters).

The vane modulation must also add a weak perturbation to the transverse field:

$$
\begin{aligned}
E_{r}= & -\frac{V}{2} \cdot r \cdot\left\{2 A_{01} \sin (k z+\phi)\right. \\
& \left.+\frac{1}{4} A_{10} k^{2}[\sin (2 k z+\phi)+\sin \phi]\right\} .
\end{aligned}
$$

The first term is the alternating-gradient focusing field, with $A_{01}$ being a geometrical focusing parameter. Like the axial perturbation the second term is weak and does not seriously modify the lateral focusing properties. However, the dependence of $A_{01} \quad(=[1$ $\left.-A_{10} I_{0}(k a)\right] / a^{2}$ ) on $A_{10}$ means that the focusing and acceleration properties must be chosen together. When $m \rightarrow 1$, then $A_{10} \rightarrow 0$ and $A_{01} \rightarrow 1 / a^{2}$, and we regain the nonaccelerating characteristics and the simple focusing field $E_{r}=-(V / a)(r / a) \times \sin \phi$ of Fig. 8 .

RFQs have replaced virtually all electrostatic preaccelerators as sources for high-energy machines, but their use to decelerate beams (Pirkl, 1997) is a relatively new application. Even in the ideal case of adiabatic deceleration (constant normalized phase-space volume) the increasing beam dimensions towards lower energies put higher demands on the rf source than in accelerating RFQs, where gentle bunching of the beam at low energies is adequate and can be achieved with modest rf fields.

The design parameters of the decelerating RFQ currently under construction for the CERN AD are shown in Table VI. Equating electric and magnetic forces, $q \mathbf{E}$ $=q \mathbf{v} \times \mathbf{B}$, shows that antiprotons at the lowest AD energy (momentum $100 \mathrm{MeV} / c$ or $\beta \sim 0.11$ ) will feel the same focusing force from the RFQ $28-\mathrm{MV} / \mathrm{m}$ electric field as would be produced by a 0.84-T magnetic field; at the RFQ output it is equivalent to $7 \mathrm{~T}$. The 250-ns-long $\mathrm{AD}$ beam will be contracted into many 5-ns bunches before entering the RFQ; after they leave it at $60 \mathrm{keV}$, an electrostatic post-decelerator will be used to adjust the particles' energy to the $10-100 \mathrm{keV}$ range. The design parameters have been chosen according to the requirements of the ASACUSA experiment (Sec. VIII.B) to collect the AD particles in a Penning trap, as described in Sec. IV.A.

\section{PRACTICAL CONDITIONS OF REST AND ISOLATION}

The idealized goal for these technological gymnastics is, we repeat, to produce samples of antiprotons at rest and in isolation, in order to be able to probe them over long periods of time in an undisturbed state. In searching for ways of achieving this, we were led to consider putting our antiprotons in cages from which they could not escape, these being constructed out of electric and magnetic fields. The cages can be either macroscopic or microscopic in nature. In the macroscopic case, they are referred to simply as traps, while in the microscopic 
TABLE VI. Design parameters of the Antiproton Decelerator Radio-Frequency Quadrupole (RFQ).

\begin{tabular}{ll}
\hline \hline Pre-buncher & \\
\hline Beam energy & $5.3 \mathrm{MeV}$ \\
Frequency & $202 \mathrm{MHz}$ \\
Effective voltage & $50 \mathrm{kV}$ \\
Drift distance to RFQ & $615 \mathrm{~cm}$ \\
Total normalized design emittance & $1 \pi \mathrm{mm} \times \mathrm{mrad}$ \\
Transverse emittance growth & $5 \%$ \\
\hline RFQ & \\
\hline Upper energy & $5.3 \mathrm{MeV}$ \\
Lower energy & $60 \mathrm{keV}$ \\
Frequency & $202 \mathrm{MHz}$ \\
Vane voltage & $167 \mathrm{kV}$ \\
Maximum electric field & $28 \mathrm{MV} \mathrm{m}{ }^{-1}$ \\
Vane length & $340 \mathrm{~cm}$ \\
Number of cells & 75 \\
Power losses & $\sim 600 \mathrm{~kW}$ \\
Average bore radius & $0.79 \mathrm{~cm}$ \\
Modulation factor $m$ (max.) & 2.9 \\
Total normalized design emittance & $1 \pi \mathrm{mm} \times \mathrm{mrad}$ \\
Total normalized acceptance & $1.5-2.0 \pi \mathrm{mm} \times \mathrm{mrad}$ \\
Transverse emittance growth & $0 \%$ \\
Longitudinal emittance growth & 1.6 \\
\hline \hline
\end{tabular}

case they are better known as exotic atoms. A third possibility is the short-term confinement of particles in extremely-low-energy particle beams.

In no case can the particles' state of isolation be absolute; the very fact that they are confined by fields implies contact or interaction between these two entities. In a trap, these contacts will be supplemented by interactions with residual gas molecules; in an exotic atom, the antiproton is continually interacting with the electron cloud and is (in nearly all cases) only imperfectly isolated from the effects of collisions of its "carrier" atom with other atoms.

The idea of rest in the "degree of motionlessness" sense also needs modification; even at $4.2 \mathrm{~K}$ in a Penning trap, the antiproton moves (and must do so in order for the trapping forces to work), while in an exotic atom it has an average orbital velocity of $\sim Z \alpha c \sqrt{m_{\bar{p}} / m_{e}}$ at capture, which increases during deexcitation of the exotic atom, as does the additional thermal motion of the atom as a whole.

Suitable working definitions of rest and isolation are always possible, however, if they are taken to mean that the particle is sufficiently motionless and sufficiently free of external contacts to allow measurement of the property being studied with the required precision. Thus rest for the ballistic-style gravitational measurements discussed in Sec. VI.C is equivalent to a few $\mu \mathrm{eV}$, this be-

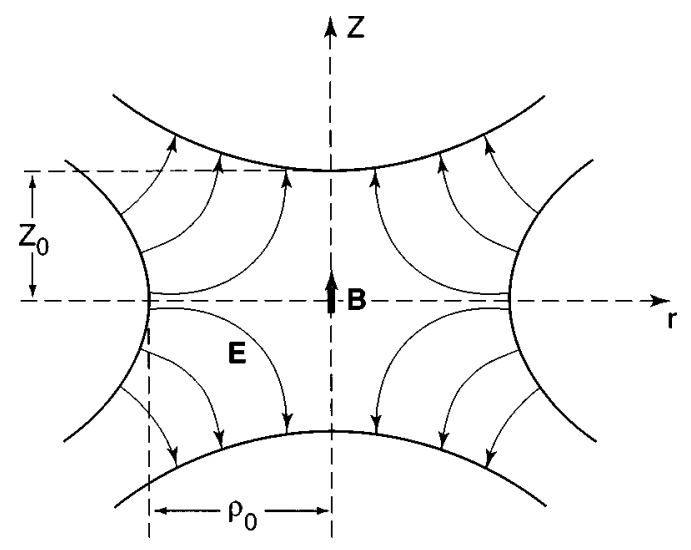

FIG. 9. Geometry and parameters of a typical Penning trap. The figure has cylindrical symmetry about the $z$ axis. All electrodes have hyperbolic section; the two on the $z$ axis (the endcap electrodes) carry a constant voltage $+V_{0} / 2$, while the remaining ring electrode carries a constant voltage $-V_{0} / 2$. Normally, particles are admitted to the trap via holes drilled in the end-cap electrodes.

ing the gravitational potential energy difference between the floor and the ceiling of a typical laboratory. Rest for measuring the $1 S-2 S$ frequency of the antihydrogen atom means that Doppler shifts and magnetic effects arising from the motion of the antihydrogen atom must be within the aimed-at experimental precision during the $1 / 7$-second lifetime of this transition and that the atom must stay within the laser-beam probe for a similar period. Likewise, isolation means the elimination of forces other than those whose interaction with the antiparticle or antiatom is being studied. Stronginteraction forces can be avoided in a particle trap simply by having a good enough vacuum to make annihilation unlikely while the measurements are being made; electromagnetic ones are avoided by ensuring that stray fields are screened out to the level at which they can be treated as small systematic corrections.

\section{A. Macroscopic traps}

All the deceleration methods described in Sec. III can produce useful samples of antiprotons with energies below a few tens of $\mathrm{keV}$. This is a suitable energy for collection and confinement by the combination of electric and magnetic fields found in charged-particle traps. In the Penning trap (Fig. 9) the particles are restricted to cyclotron orbits in the $x-y$ plane by an axial ( $z$ ) magnetic field, while a superimposed electric quadrupole field forms a potential well around them that further constrains them to harmonic (and therefore momentumindependent) motion along the $z$ axis.

Brown and Gabrielse (1986) have given a very thorough review of the properties of charged-particle traps, with particular emphasis on those used for confining antiprotons. The reader is referred to this excellent paper for a comprehensive treatment of the material summarized in the next few paragraphs. 


\section{Loading antiprotons into traps}

Particles entering a trap will sooner or later find their way out again since in the absence of dissipative forces they must eventually return to the aperture through which they entered it-usually a hole in one of the electrodes. The simplest way around this problem is to bunch the particles before entry and to switch on the voltage of the electrode via which they entered (one of the axially symmetric ones for the case of Fig. 9) only after the bunch is inside.

Thus if a thin foil used for passive deceleration as described above forms part of an upstream trap electrode (Kalinowsky, 1993), it can be raised in potential once the bunch emerging from the foil has fully entered the trap structure. The pioneering experiments on antiproton trapping were done by the Harvard group led by Gabrielse (Gabrielse et al., 1986). In these experiments, a 300- $\mu \mathrm{m}$ entrance window was used with titanium and Mylar foils and a $\mathrm{SF}_{6}$ gas cell as a fine-tunable moderator. The purpose of these experiments was to make a precision comparison of the $\mathrm{e} / \mathrm{m}$ value of protons and antiprotons (discussed in Secs. VI.B.1 and VI.B.3 below) by measuring the cyclotron frequencies of single trapped particles, and not to accumulate large numbers of them. Consequently a shallow trap of depth $3 \mathrm{kV}$ was used in these first experiments, and the efficiency for capture into it was only $1.6 \times 10^{-4}$. In later experiments, the same group focused on sequential loading of many bunches with the long-term view of antihydrogen production (Gabrielse et al., 1993) and succeeded in accumulating $\sim 10^{6}$ antiprotons in a similar structure. $M$. Holzscheiter's group (1992) was able to accumulate a similar number of 5.6-MeV antiprotons from LEAR in a 30-kV-deep Penning trap after degrading their energy in a number of thin windows and moderators. Calculations based on the TRIM program (Ziegler, 1991) give a maximum theoretical transmission of $2.5 \%$ of the beam antiprotons into this energy window. So far this figure has not been achieved, the best figure being, for unknown reasons, of the order of $0.4 \%$.

In the cyclotron trap the deceleration is normally continued in the gas down to some tens of electron volts, at which exotic atoms are formed (see Sec. IV.B.2). However, it should also be possible to interrupt this process at a few tens of $\mathrm{keV}$ and remove the particles from the cyclotron magnet center into an ultrahigh vacuum via an axial borehole equipped with a very thin foil window (Eades and Simons, 1989). This requires an extremely low gas pressure, and attempts have been made to reduce the low-pressure losses on injection (mentioned in Sec. III.B.2 and shown in Fig. 7) by using a pulsed electrostatic inflector following the entrance window (Aschenauer et al., 1992). Extraction from the cyclotron trap center has indeed been successfully demonstrated, so far only with $\mu^{-}$and using a somewhat different deceleration technique (DeCecco et al., 1997). The highest number of extracted muons was $2 \times 10^{4} / \mathrm{s}$ for $10^{8}$ injected pions, and a figure for antiprotons can be estimated by correcting these figures for the $\pi$ and $\mu$ decays during deceleration and extraction of the low-energy muons. When this is done, the estimated efficiency is $20 \%$ for antiprotons, though only $6 \%$ has so far been achieved in the LEAR antiproton beam (Kalinowsky, 1993).

Finally, the RFQ under design for the Antiproton Decelerator should achieve a transmission efficiency of about $50 \%$. A continuous vacuum system between the $\mathrm{AD}$ and a trap at its exit should result in a loading efficiency close to this value.

\section{Motion in traps}

Once inside the trap, the antiproton sample moves according to the equations of motion imposed by the applied fields. For a Penning trap the magnetic field $B$ (along $z$ ) is usually highly uniform. The motion of particles of charge $q$ and mass $m$ in the $x-y$ plane $\left(\rho^{2} \equiv x^{2}\right.$ $\left.+y^{2}\right)$ is then close to circular, with the cyclotron orbit frequency

$$
\omega_{c}=(q / m) \cdot B
$$

When harmonic $z$ motion is required, a voltage $V_{0}$ applied between hyperboloid-of-revolution electrodes as in Fig. 9 will produce the required quadrupole potential:

$$
V=V_{0} \times \frac{z^{2}-\rho^{2} / 2}{d^{2}},
$$

where $d^{2} \equiv z_{0}^{2}+\rho_{0}^{2} / 2$ (see Fig. 9). The simple harmonic axial motion then has frequency

$$
\omega_{z}=\left(\frac{q V_{0}}{m d^{2}}\right)^{1 / 2} .
$$

The motions under the combined electric and magnetic fields are not independent, their interaction moving the cyclotron orbits away from the axis and adding magnetronlike motion of this eccentric orbit in the $x-y$ plane with frequency

$$
\omega_{m} \sim \omega_{z}^{2} / 2 \omega_{c}
$$

Normally the binding of the particles to the electric quadrupole field is a weak perturbation on the magnetic binding, and $\omega_{m} \ll \omega_{c}$.

In the Penning-Malmberg variant of the Penning trap (Malmberg and de Grassie, 1975; Malmberg and Driscoll, 1980), the hyperboloid-of-revolution electrode geometry is deformed into a sequence of cylinders along the $z$ axis. With this geometry, axial harmonicity can be retained over much of the trap volume by a suitable choice of the length/radius ratio of the cylinders. One of the solutions proposed for collecting antiprotons and positrons for antihydrogen formation at the AD is a Penning-Malmberg trap, whose characteristics are summarized in Table VII.

Other cylindrical electrode arrangements result in flat-bottomed or square-well traps. These have no harmonic properties, although a harmonic region can be superimposed near $z=0$, if required, by adding further short axial cylinders. 
TABLE VII. Some parameters of a typical trap.

\begin{tabular}{ll}
\hline \hline Trap length $\ell$ & $50 \mathrm{~cm}$ \\
Trap diameter & $2 \mathrm{~cm}$ \\
Trapping voltage $V$ & $15 \mathrm{kV}$ \\
Trapping efficiency & $\sim 1$ \\
Magnetic field $B$ & $2.5 \mathrm{~T}$ \\
$\Delta B / B$ & $10^{-3}$ \\
Effective duration of trapped pulse & $200 \mathrm{~ns}$ \\
\hline \hline
\end{tabular}

\section{Deceleration and cooling in traps}

Our trapped sample of antiprotons is now isolated from walls and other manifestations of ordinary matter by the joint effect of the trap fields and the ultrahigh vacuum. Were this isolation complete, there could be no further interchange of energy between them and the rest of the universe.

In practice there are two unavoidable points of contact between the antiprotons and the outside world, which will ensure that these will eventually cool from tens of $\mathrm{keV}$ to thermal equilibrium with the trap walls (usually at liquid-helium temperature, $4.2 \mathrm{~K} \sim 0.36 \mathrm{meV}$ for superconducting traps). These are (a) the synchrotron radiation associated with the nonrectilinear antiproton motion in the trap and (b) the movement of charges induced by the moving antiproton sample in the trap electrodes (the latter normally being coupled to external circuitry). The first and second laws of thermodynamics alone will therefore eventually bring the sample to the ambient energy. Neither process, however, represents a practical cooling mechanism. Synchrotron radiation for antiprotons is extremely slow; Wille (1991) gives the power radiated by a particle of orbital radius $\rho$ as

$$
P=\frac{1}{6 \pi \epsilon_{0}} \frac{e^{2} c}{\rho^{2}} \beta^{4} \gamma^{4} \sim 2874 \frac{\beta^{4}}{(\rho / \mathrm{cm})^{2}}\left[\mathrm{eV} \mathrm{s}^{-1}\right] .
$$

For mass $m_{\bar{p}}$ in a field $B$ that is uniform along $z$ (as is approximately true in most trap configurations), we can write $\rho=m_{\bar{p}} \beta_{t} c /(e \cdot B)$ for the transverse motion and get

$$
\frac{d T_{\perp}}{d t}=-P=-\frac{1}{3 \pi \epsilon_{0}} \frac{e^{4} B^{2}}{c^{3}} \times \frac{T_{\perp}}{m_{\bar{p}}^{3}} .
$$

The transverse kinetic energy therefore falls exponentially with lifetime:

$$
\tau_{\mathrm{rad}}=3 \pi \epsilon_{0} \frac{c^{3}}{e^{4} B^{2}} \times m_{\bar{p}}^{3},
$$

so that, for the same kinetic energy, radiative deceleration is $1840^{3}$ times less efficient for antiprotons than for electrons or positrons. For $T=20 \mathrm{keV}$ and typical trap fields of $B=5 \mathrm{~T}, \rho=4 \mathrm{~mm}, P \sim 3.1 \times 10^{-5} \mathrm{eV} \mathrm{s}^{-1}$, and $\tau_{\text {rad }}=6.2 \times 10^{8} \mathrm{~s}$.

Brown and Gabrielse (1986) describe how a refrigerated, but purely resistive, element $R$ connected to the trap electrode and driven by the axial motion of the antiprotons can couple axial energy out of the antiproton cloud via induced currents. According to Walls and Dehmelt (1968), the time constant for this is

$$
\tau_{R}=\left(\frac{2 z_{0}}{e \kappa}\right)^{2} \frac{m_{\bar{p}}}{R}
$$

where $z_{0}$ is the characteristic axial dimension of the trap and $\kappa$ a dimensionless geometrical constant of the trap of order 1 (Gabrielse et al., 1995). For a trap with $z_{0}$ $=1 \mathrm{~mm}$, and $R=10^{5} \Omega$ the resistive cooling time would be of the order of one hour. A formula similar to Eq. (14) but with the trap's radial parameter replacing the axial one gives a time constant of the same order for resistive damping of the cyclotron motion (Brown and Gabrielse, 1986).

For both radiative and resistive cases, methods exist to improve the cooling/deceleration efficiency. Thus if an electron cloud is added to the trap, it will receive energy from the antiprotons by thermal mixing. According to Eq. (13) with $m_{e}$ substituted for $m_{\bar{p}}$, this energy will be reradiated with a time constant $\sim 2.6 / B^{2} \mathrm{~s}(B$ in tesla). With $\mathcal{O}(1840)$ electrons per antiproton, the mixing becomes so efficient that the antiprotons appear to radiate at almost the electron rate (Beck et al., 1992, 1996). Such procedures are clearly variations on the electron cooling methods used in storage rings. ${ }^{11}$

Gabrielse et al. $(1989,1990)$ have demonstrated electron cooling on a sample of antiprotons trapped at $\sim 3 \mathrm{keV}$ in a 6-T field. With $10^{7}$ electrons losing energy partly by radiation and partly via their coupling to the resistive part of a 4.2-K resonant circuit connected to one of the trap electrodes, $\sim 10^{4}$ antiprotons were cooled to $10^{-4} \mathrm{keV}$ over a period of $10 \mathrm{~s}$.

Other methods suggested for cooling antiprotons include a stochastic technique analogous to that used in storage rings (Beverini et al., 1988a, 1988b; Rolston and Gabrielse, 1988) and the adiabatic expansion of the antiproton cloud via slow reduction of the trap electrode voltages (Li et al., 1993).

\section{B. Microscopic traps: exotic atoms}

In referring to the exotic atom as a microscopic trap for the antiproton, we are casting a wide and independent field of research in a rather unusual light. The many similarities between exotic atoms and macroscopic traps make this a valid and useful point of view, but there are also several important differences. The most important of these is the fact that the trap provided by the antiprotonic atom for the $\bar{p}$ is far from ideal: the lifetime in it is short (of the order of ns, in one very favorable case described in Sec. IV.B.5 below, in the $\mu$ s region), the antiproton interacts with the electrons and the nucleus of the host atom, and the atomic trap itself (i.e., the carrier atom) is far from being really isolated even in

\footnotetext{
${ }^{11}$ Another well-known technique of a similar nature is cooling by collisions with buffer gas molecules (Greaves et al., 1994). This is out of the question for antiprotons, which would first be captured by the gas molecules and then annihilate, but as we shall see in Sec. VII.A, the method can be used for cooling positrons, whose annihilation rate for suitable gas densities is one order of magnitude slower than the cooling rate.
} 
dilute gases. Nevertheless, even a nanosecond lifetime corresponds to many orbital periods. Antiprotonic atoms are therefore extremely well-defined objects, the study of which has brought valuable information on such fundamental quantities of the antiproton as its mass, charge, and magnetic moment; considerably more can be expected in the coming era of the AD. In our treatment below we accompany the antiproton closely on its journey as it slows down below the maximum of the $d E / d x$ curve via atomic collisions, displaces a single electron from the host atom in which it takes up residence, interacts with the other inhabitants of its microscopic trap (the host atom's remaining electrons), and finally annihilates in this atom's nucleus.

\section{Slowing down below the Bragg peak}

Before the $\bar{p}$ reaches energies of a few tens of $\mathrm{eV}$, the upper limit of the capture region, it has to be slowed down by a further factor of a 1000 or more from the energy regime discussed in Sec. III.B. The theoretical and experimental basis for the stopping power in this energy region is by far less solid than that in the BetheBloch regime: the contribution of the various atomic electrons to the stopping process varies drastically with energy, the polarization of the medium by the heavy charged particle becomes more and more important, and, finally, nuclear stopping, i.e., the energy loss by elastic collisions with the atomic nucleus, comes into play.

This is not the place to give a full account of the stopping of charged particles at low energies; a rather recent review may be found in the ICRU Report 49 (1993). A few facts that are relevant to capture in exotic atoms (and also in traps) must, however, be mentioned.

Already in their first studies of the approach to capture, Fermi and Teller treated the electrons in a moderator as a Fermi gas. The stopping power $S$ at energies well below the Bragg peak was predicted to be proportional to the particle velocity (Fermi and Teller, 1947). Since this fundamental work many other authors (see ICRU Report 49, 1993) have corroborated this result, although some experiments reported deviations for protons at very low energies (Golser and Semrad, 1991). The dominant role played by nuclear stopping at the lowest energies (below $1 \mathrm{keV}$ for $\bar{p}$ in hydrogen) is evident in the data produced for hydrogen by the OBELIX collaboration at LEAR (Fig. 10; Agnello et al., 1995)

Several aspects of the deceleration process are especially important in calculations of the capture efficiency of antiprotons in traps and of their stopping efficiency in the low-density gas targets normally used in studies of exotic-atom processes.

First of all, the very effect which blows up the energy distribution of the beam above the Bragg peak works to narrow it at energies below it (Daniel, 1992), because in this region, $S$ (which has the dimensions of force) decreases with decreasing energy and therefore opposes the motion of fast particles more strongly than that of slow ones. This phase-space compression effect has re-

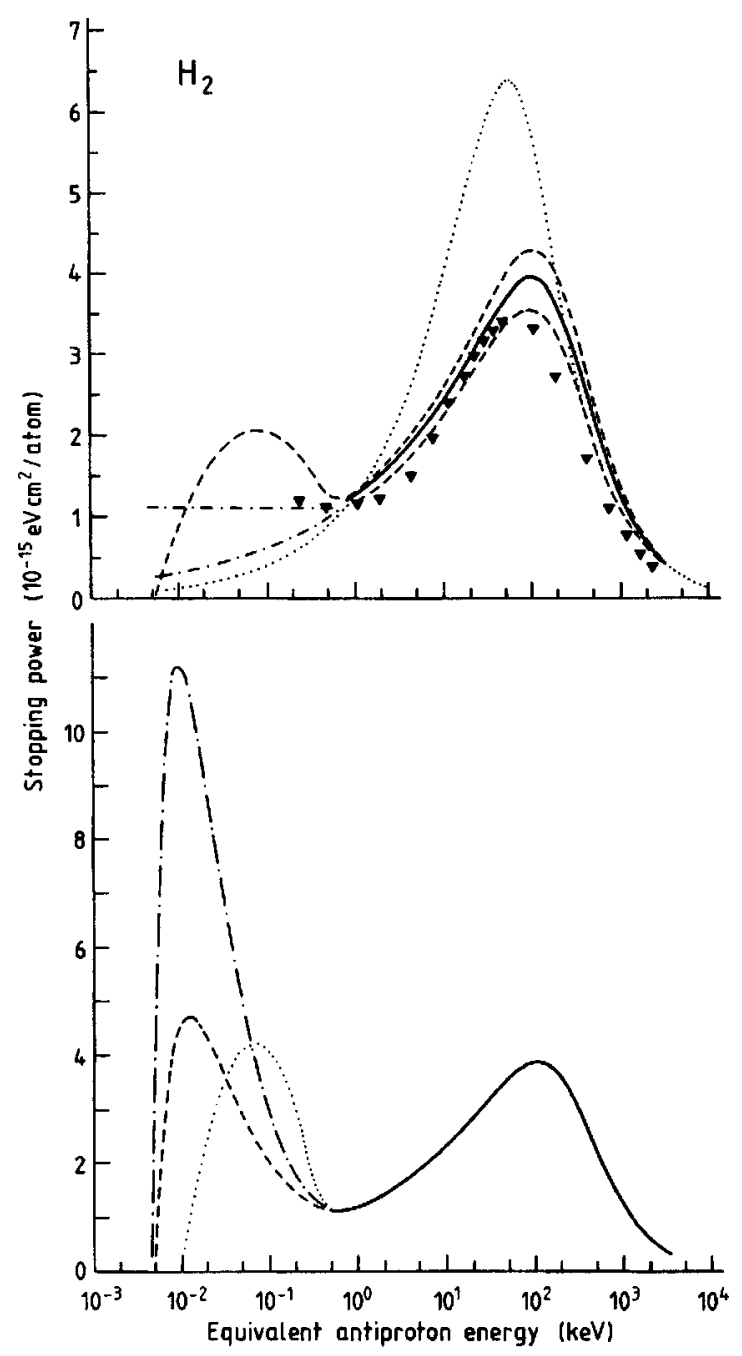

FIG. 10. Stopping power for low-energy antiprotons in $\mathrm{H}_{2}$ (Agnello et al., 1995). Upper part: Best fit (solid line) with upper and lower limits (dashed lines). The solid triangles denote $\mu .^{-}$data (Hauser, 1993), the dotted lines proton data. Lower part: Three acceptable paths of the $\bar{p}$ stopping power below $0.5 \mathrm{keV}$ (dashed, dotted, and dash-dotted lines). Solid line: fit to the data.

cently been proven in frictional cooling experiments with $\mu^{-}$(Mühlbauer et al., 1996). By slowing down the exotic particles in a stack of thin foils, and accelerating them in between with the help of a strong axial electric field, the phase-space volume of a $10-\mathrm{keV}$ muon beam could be decreased by a factor of ten; this factor came about partly because the axial field turns the velocity vector towards the beam axis and thus decreases the beam divergence.

At very low energies, multiple scattering effects (Molière, 1947; Marion and Zimmerman, 1967) will be even more serious than in the energy region above the Bragg peak, possibly leading even to backscattering out of the target.

Antiproton annihilation is, at least for light elements, a less serious problem during deceleration than one might expect. At low energies, the annihilation cross section is expected to rise rapidly with decreasing energy 
$\left[\beta^{2} \cdot \sigma_{\text {ann }} /\right.$ mbarn $\approx 0.51$ for hydrogen in the low-energy limit (Carbonell and Protasov, 1993)]. Annihilation nevertheless remains an unlikely outcome (relative to energy loss) of the antiproton's encounter with the atom down to the $100-\mathrm{eV}$ region, as it must score a direct hit on the nucleus to annihilate, but need only strike the atom to lose energy. Whereas the cross section for energy loss is in the $10^{-16} \mathrm{~cm}^{2}$ region for energy transfers of around $10 \mathrm{eV}$ per collision, the annihilation cross section is below $10^{-20} \mathrm{~cm}^{2}$, even at $100 \mathrm{eV}$.

All these effects can usually be taken suitably into account when modeling the capture process.

\section{The formation of antiprotonic atoms}

The Coulomb capture process (in which the antiproton changes places with a single atomic electron) begins when the $\bar{p}$ kinetic energy has been reduced to a few tens of eV. From this energy downward the probability for capture, which rises exponentially with decreasing energy (Cohen, 1983), reaches the order of magnitude of the probability for further energy loss. Two views of the capture process have become familiar, the semiclassical and the fully quantum-mechanical picture.

In the semiclassical treatment the antiproton is assumed to move on trajectories determined by classical mechanics; this seems well justified as the de Broglie wavelength of a $10-\mathrm{eV}$ antiproton, for example, is only $0.009 \mathrm{~nm}$, whereas the orbital radius of the $\mathrm{K}$ electrons in, for example, $\mathrm{Ca}$ is $0.41 \mathrm{~nm}$ (Bunge et al., 1993). The description of the $\bar{p}$ interaction with the atomic or molecular electrons, however, is based on quantummechanical calculations with the atomic electrons usually assumed to be a degenerate electron gas; for the lightest elements the kinematics of the individual electrons may be calculated directly. Although few calculations have been performed for antiprotons (Beck et al., 1993; Cohen, 1997), the results for muons can easily be transferred to $\bar{p}$ by appropriate kinematic scaling factors.

A brief description of the semiclassical picture of the Coulomb capture process is as follows. The approaching antiproton feels the screened Coulomb potential of the nucleus and, depending on the impact parameter at encounter, is deflected more or less strongly towards the center of the atom. It gains kinetic energy in the screened electric potential of the nucleus and loses it by interaction with the electrons. The angular momentum barrier, which every particle approaching an atom with finite impact parameter experiences, also plays an important role in the capture process; it can stop the approaching $\bar{p}$ from penetrating the atomic-collision partner, but may also trap a particle which has penetrated the atom, lost energy inside it, and therefore sees the barrier from the other side. As the $\bar{p}$ velocity before capture is much lower than that of the atomic electrons, the response of the electron shell to the heavy, slow projectile has to be taken into consideration. A first step in doing this is to include a polarization term (Leon and

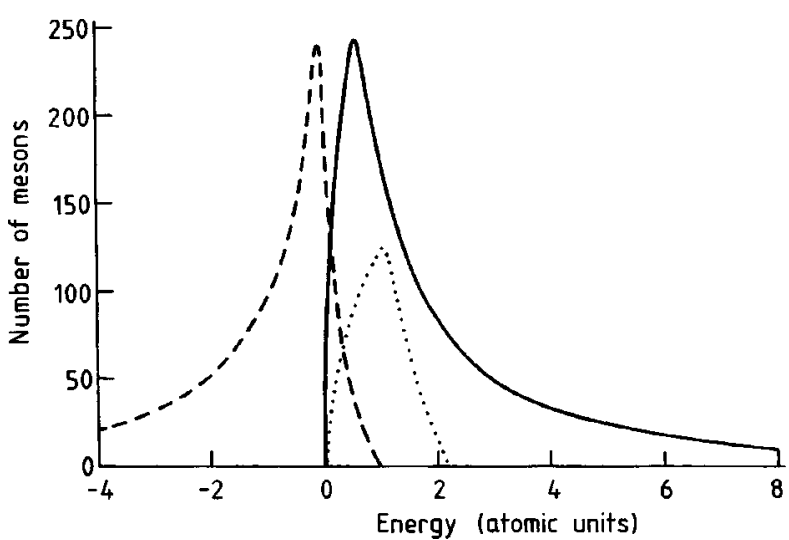

FIG. 11. Energies before and immediately after Coulomb capture of muons in $\operatorname{Zr}(Z=40)$ : solid line, before; dashed line, after; dotted line, result of the Fermi-Teller model for the distribution of energies immediately before capture (Leon and Miller, 1977).

Seki, 1977) $U(r) \propto-\alpha[1-\exp (-r / 2)] /\left(4+r^{2}\right)^{2}$, with $\alpha$ the atomic polarizability, in the interaction potential.

The author list for semiclassical calculations is rather long (Leon and Seki, 1974, 1977; Daniel, 1975, 1979, 1980, 1981; Vogel et al., 1975, 1977; Leon and Miller, 1977). One characteristic example is that of Leon and Miller, who find the distribution of muon energies immediately before and after capture (Leon and Miller, 1977) shown in Fig. 11; here again the conditions for muons may easily be transferred to antiprotons. The authors also obtain an angular momentum distribution after atomic capture which is very close to the statistical one $[p(\ell) \propto 2 \ell+1]$ predicted by simple considerations such as the $(2 \ell+1)$-fold multiplicity of a level with angular momentum quantum number $\ell$. In contrast Daniel (1981) deduced, from the shape $n(W)$ of the energy spectrum of the exotic particle before capture and from the distribution of impact parameters, an initial angular momentum distribution that is low for both low and high angular momenta, and peaks at $\ell$ values depending on the form of $n(W)$ (cf. Fig. 12). Experimental findings from muonic atoms indicate that the angular momentum distribution $p(\ell)$ may even, for the transition and rare-earth elements, be nearly constant. There are now reasons to believe that the initial distribution given in Fig. 12 is more accurate.

This angular momentum distribution determines the fraction of antiprotons that cascade down to the lowest levels of the exotic atom: As the $\bar{p}$-nucleus interaction is strong for the levels with small $\ell$, even at very large $n$ (West, 1958), flat angular momentum distributions mean large antiproton losses by annihilation almost immediately after capture in the exotic-atom trap.

The Fermi-Teller view of the atomic electrons as a degenerate Fermi gas, mentioned above, implies that the semiclassical calculations are not suited for atoms with low $Z$. It is just these atoms, however, that are especially interesting as microscopic traps in future experiments. A number of calculations have been performed on the atomic capture of exotic particles by $\mathrm{H}$ and $\mathrm{He}$. The 


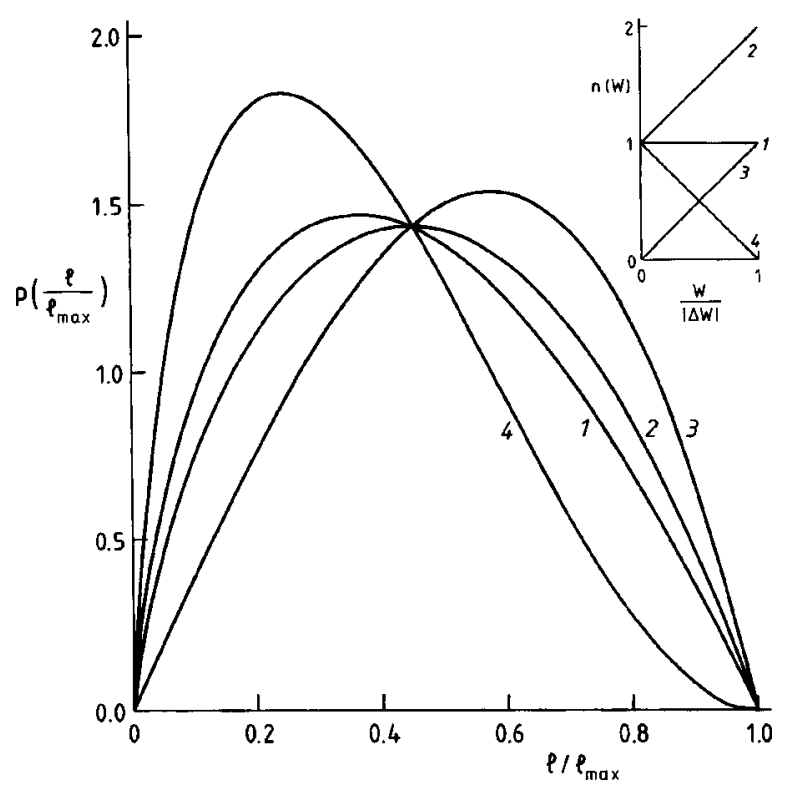

FIG. 12. Initial angular momentum distribution $P\left(\ell \mid \ell_{\max }\right)$ of muons after Coulomb capture under various assumptions for the kinetic-energy spectrum before capture. The numbers labeling the different $\ell$ distributions apply to the corresponding kinetic-energy distributions $n(W)$ in the insert (Daniel, 1981).

most recent include a calculation based on quantum coupling equations performed by Dolinov et al. (1989) and two quasiclassical approaches: Beck et al. (1993) have developed a theory for the atomic capture of $\bar{p}$ in He and very recently Cohen (1997) devised a corresponding theory for $\bar{p}$ capture in hydrogen molecules. Results from this calculation for the initial $\ell$ distribution in hydrogen are shown in Fig. 13.

The development of fully quantum-mechanical theories is hindered by the complexity of the description of the electron shell for all atoms with $Z>2$. Hence calculations of this kind have been nearly exclusively for muons in hydrogen and helium (a survey may be found in Hartmann, 1990). They agree in predicting that capture takes place only at energies below $100 \mathrm{eV}$ and that the initial $\ell$ distribution is close to the statistical one.

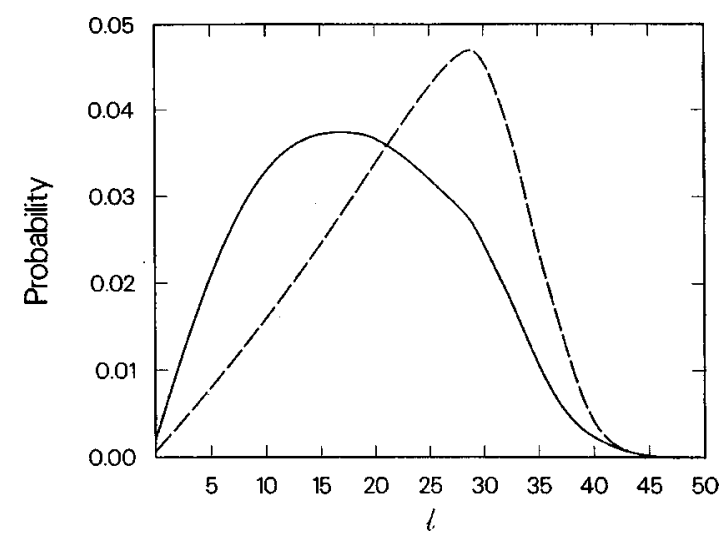

FIG. 13. Initial angular momentum distribution of antiprotons after Coulomb capture in hydrogen; solid line, hydrogen molecules; dashed line, hydrogen atoms (Cohen, 1997).
Confrontations of these theoretical results with experimental data on the Coulomb-capture process are, except for $\bar{p}$-He, quite scarce. This is certainly because determination of the extremely low energy of the antiproton immediately before capture, and the detection of the radiation or Auger electrons, again of low energy, emitted after it, is experimentally very difficult. A favorable exception is the antiprotonic helium atom. Systematic studies of transition energies and populations of this atom have widened our view on capture and cascade (Morita et al., 1994; Hayano et al., 1994; Maas et al., 1995), as will be described in Sec. IV.B.5. At least for this exotic atom, the long-held but intuitive picture that immediately after capture the radius of the antiproton orbit is roughly the same as that of the displaced electron (principal quantum number $n_{\mathrm{e}}$ ) has been confirmed, the expected value of the antiproton's principal quantum number, $n \approx \sqrt{m_{\text {red }} / m_{\mathrm{el}}} \cdot n_{\mathrm{e}} \approx 38$ for $\bar{p}-{ }^{4} \mathrm{He},{ }^{12}$ having now been found experimentally. This suggests that antiprotons captured by elements with higher $Z$ should have very high initial principal quantum numbers. For capture by $L$-electron emission in argon, for example, one must expect that $n>100$.

\section{The structure of antiprotonic atoms}

Our antiproton has now been loaded into its exoticatom trap and is orbiting the atomic nucleus, alone (in the case of protonium) or in the company of the remaining atomic electrons. What is the nature of this new abode? For nearly all relevant atomic levels, the antiproton's orbit will lie within or near the electrons' $\mathrm{K}$ shell for any atom. By Gauss's theorem these $Z-1$ remaining electrons should have no effect on the antiproton's motion and the point-nucleus binding energy of the antiparticle for a level with principal quantum number $n$ and total-angular-momentum quantum number $j=\ell \pm 1 / 2$ will be the Dirac result

$$
E_{n j}=m_{\mathrm{red}} c^{2}\left[\left[1+\left(\frac{Z \alpha}{n+\epsilon_{j}}\right)^{2}\right]^{-1 / 2}-1\right]
$$

with $\epsilon_{j}=\left[\left(j+\frac{1}{2}\right)^{2}-(Z \alpha)^{2}\right]^{1 / 2}-\left(j+\frac{1}{2}\right)$. The dependence of Eq. (15) on $m_{\text {red }}$ means that measurement of antiproton transition energies will determine the $\bar{p}$ mass (or more accurately the Rydberg constant). A number of corrections have to be applied, however, to this simple picture:

(1) The finite size of the nucleus manifests itself in a reduction of the binding energy. If the shape of the nucleus is known, the Dirac equation for the finiterange electrostatic potential may be solved numerically.

(2) The anomalous magnetic moment of the antiproton has to be taken into account by the addition of a Pauli term in the Dirac equation (Borie, 1983).

\footnotetext{
${ }^{12}$ Here and elsewhere, $m_{\text {red }}$ denotes the reduced mass of the $\bar{p}$ in the atom.
} 
TABLE VIII. Contribution to the binding energies of high-lying levels in antiprotonic ${ }^{208} \mathrm{~Pb}$ (all values in $\mathrm{keV}$ ).

\begin{tabular}{cccccc}
\hline \hline $\begin{array}{c}\text { Level } \\
(n, j)\end{array}$ & $\begin{array}{c}\text { Point } \\
\text { nucleus }\end{array}$ & $\begin{array}{c}\text { Vacuum } \\
\text { polar. }\end{array}$ & $\begin{array}{c}\text { Electron } \\
\text { screen. }\end{array}$ & $\begin{array}{c}\text { Other } \\
\text { corr. }\end{array}$ & $\begin{array}{c}\text { Final } \\
\text { energy }\end{array}$ \\
\hline $8,15 / 2$ & 2615.721 & 12.926 & 0.024 & 0.200 & 2628.871 \\
$8,13 / 2$ & 2617.820 & 12.960 & 0.023 & 0.201 & 2631.004 \\
$10,19 / 2$ & 1673.216 & 6.220 & 0.047 & 0.100 & 1679.583 \\
$10,27 / 2$ & 1673.883 & 6.229 & 0.047 & 0.100 & 1680.259 \\
$11,21 / 2$ & 1382.608 & 4.472 & 0.063 & 0.054 & 1387.197 \\
$11,19 / 2$ & 1383.018 & 4.477 & 0.063 & 0.053 & 1387.611 \\
$12,23 / 2$ & 1161.637 & 3.272 & 0.082 & 0.012 & 1165.003 \\
$12,21 / 2$ & 1161.890 & 3.274 & 0.082 & 0.022 & 1165.268 \\
\hline \hline
\end{tabular}

(3) A vacuum polarization correction will arise from the generation of $e^{+} e^{-}\left(\mu^{+} \mu^{-}\right)$pairs in the field of the nucleus. This, the most important radiative correction to the binding energy, has to be calculated to all orders because the range of the resulting potential is of the order of the electronic Compton wavelength (Borie, 1983). Further corrections of minor importance are those for relativistic recoil (the introduction of a reduced mass is only correct for the nonrelativistic problem) and for the polarization of the nucleus by the antiproton.

(4) The antiproton orbit will not always be fully inside the $\mathrm{K}$ shell. The (small) part of the electron cloud between particle and nucleus leads to a screening of the nuclear charge and changes the binding energy of the $\bar{p}$, for loosely bound levels quite drastically. The combined solution of the Dirac equation for nuclear and electron potential is much too complicated and approximation techniques have to be used. Vogel (1974) gives an account of the calculation procedure and presents numbers (although only for muonic atoms).

(5) The interaction of the $\bar{p}$ magnetic moment with that of the nucleus splits the levels. This splitting has been used to determine the orbital magnetic moment of the antiparticle (Sec. VI.B.4).

(6) The (short-range) strong interaction with the nucleus plays a decisive role for the levels with low $\ell$ because it not only shifts the binding energy for low $n$, but permits the disappearance of the antiproton even at high $n$ (Sec. IV.B.2). Thus annihilation may diminish the supply of antiprotons so urgently needed for good-statistics measurements. When one scales the annihilation width of the $\bar{p}-\mathrm{Ca}(n=1, \ell$ $=0)$ level, $\Gamma_{1,0}=943 \mathrm{keV}$, according to the overlap of the antiproton wave function with the nucleus to the $(n=15, \ell=0)$ level (West, 1958), the resulting width, $\Gamma_{15,0}=279 \mathrm{eV}$, is still orders of magnitude larger than the radiative width, $\Gamma_{\mathrm{rad}}=0.06 \mathrm{eV}$.

Table VIII gives an overview of the point-nucleus binding energy (including the effect of the anomalous magnetic moment) and the various correction terms for the case of $\bar{p}-\mathrm{Pb}$. As may be seen from this table, the corrections for levels with adjacent $n$ are very similar.
This means that most of them cancel when measuring transition energies and that experiments to derive, for example, the $\bar{p}$ mass and magnetic moment have a good chance of success.

\section{The antiprotonic-atom cascade}

In the exotic-atom cascade of the $\bar{p}$ from the highly excited states populated at capture down to the levels from which annihilation takes place, it is possible to discern rough analogues of cooling and deceleration processes in macroscopic traps (Sec. IV.A.3). Thus radiative transitions in the Coulomb field correspond to synchrotron radiation, while interaction with the atom's electrons correspond to "cooling" in an electron gas. The latter interaction is better known as the internal Auger effect; where this is energetically possible it involves the deexcitation of the atom with emission of the atomic electrons.

Two crucial differences are that the force $q E$ ( $E$ $=d V / d r)$ on the antiproton increases monotonically with decreasing $r$ in the exotic atom, so that it accelerates instead of decelerating, and that the spontaneous processes, which were very slow in the macroscopic case, are extremely rapid in exotic atoms for elements heavier than He. Even for these, the cascade time is several hundred orbital periods; the concept of an exotic atom is thus perfectly well defined and our description of it as a microscopic trap retains its validity.

In the early phase of the cascade, the antiproton may be viewed-as in the semiclassical treatment of Coulomb capture-as a classical particle moving in the (shielded) field of the nucleus, losing energy and angular momentum by interacting with the degenerate Fermi gas of the atomic electrons and by emitting bremsstrahlung. Calculations by Rook (1970) indicate that in this part of the cascade the Auger effect dominates, and the shape of the angular momentum distribution remains unchanged.

For principal quantum numbers $n<30$ the $\bar{p}$ is well inside the electronic $\mathrm{K}$ shell and the influence of the electron cloud may be neglected. The quantummechanical calculation for such hydrogenlike exotic atoms is quite straightforward, and all relevant formulas 


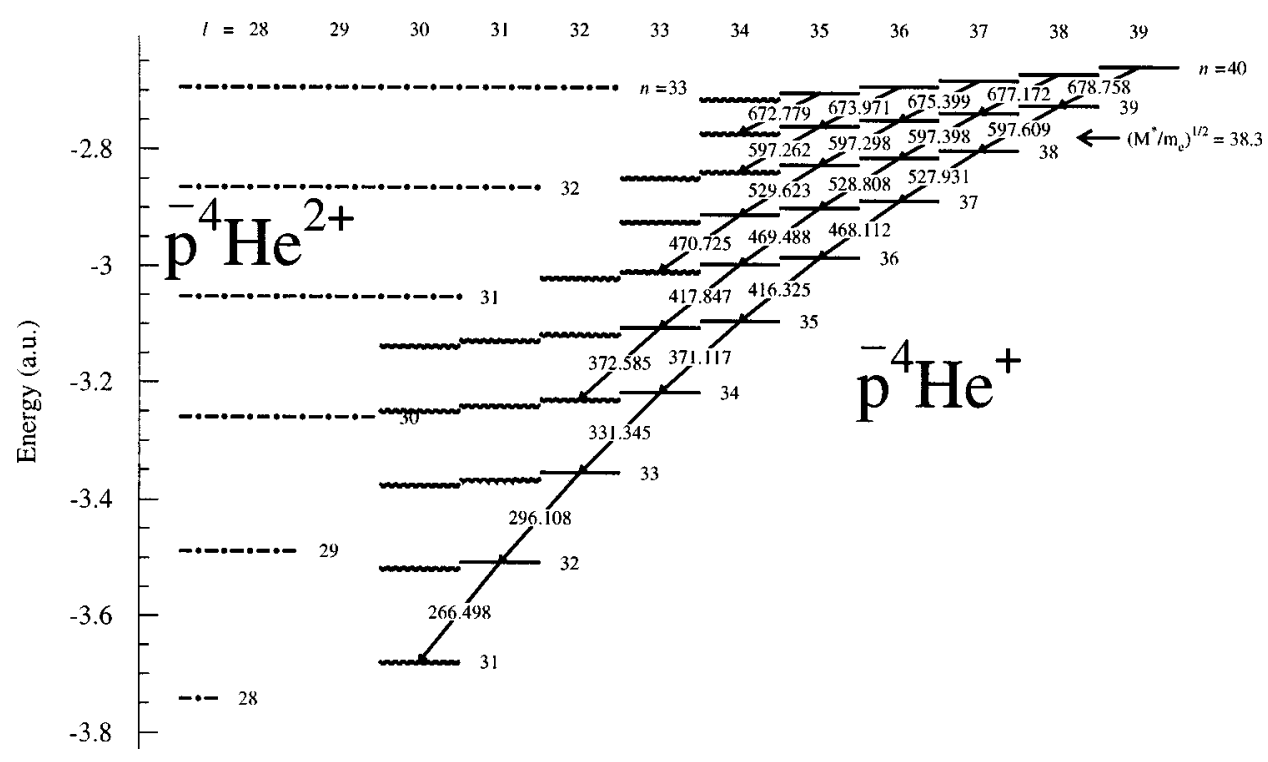

FIG. 14. Scheme of high-lying levels in $\bar{p}-{ }^{4} \mathrm{He}$. The numbers on the arrows denote the transition wavelength in $\mathrm{nm}$.

may be found in the thesis of Akylas (1978). The rate for radiative transitions of multipolarity $\lambda$ is given by

$$
\Gamma_{\mathrm{rad}}^{\lambda}=\mathrm{const}\left\langle f\left|r^{\lambda}\right| i\right\rangle E^{2 \cdot \lambda+1} .
$$

The most important transitions are the lowest-order electric ones; Akylas and Vogel (1978) showed that the rate for higher-order transitions is suppressed by a factor

$$
\Gamma_{\mathrm{rad}}^{\lambda+1} / \Gamma_{\mathrm{rad}}^{\lambda} \leqslant\left[\frac{Z \cdot \alpha}{n}\right]^{2}
$$

which for an antiprotonic atom with $Z=40$ and at $n$ $=10$ is a factor of about $10^{-3}$. The formula for the rate of electric dipole transitions becomes especially simple for those between circular orbits $(\ell=n-1)$ (Scheck, 1983):

$$
\begin{aligned}
\hbar \cdot \Gamma(E 1 ; n \rightarrow n-1) \\
\quad=\alpha^{5} \cdot m_{\mathrm{red}} c^{2} \cdot Z^{4} \frac{2^{4 n} n^{2 n-4}(n-1)^{2 n-2}}{3(2 n-1)^{4 n-1}},
\end{aligned}
$$

where $m_{\text {red }}$ is the reduced $\bar{p}$ mass.

The treatment of Auger transitions is somewhat more complicated because all multipole orders up to the octupole have to be included, and the dynamics of the antiproton-electron interaction may play a role (Leon and Seki, 1978).

The antiprotonic cascade is a complicated interplay between radiative and Auger transitions among a continuously changing group of atomic electrons. These electrons may be refilled from the outside in the case of metals or even insulators (the sometimes highly charged exotic atom strongly attracts electrons from the surroundings), but in low-pressure noble gases this supply is far too slow to prevent complete depletion by the continuous loss of electrons (Bacher et al., 1988). Completely stripped antiprotonic atoms constitute ideal traps for the $\bar{p}$, albeit for a very short time. In the absence of an electron cloud, the study of antiprotonic atoms should permit investigation of such fundamental $\bar{p}$ properties as mass and magnetic moment, as these enter directly into the formulas for the binding energy, as indicated above.

As we have suggested, the atomic cascade terminates when the antiproton annihilates on a proton or neutron of the nucleus, with the characteristic strong-interaction time of $10^{-23} \mathrm{~s}$. Except for the lightest elements, this occurs well before the antiproton has reached its $(1 S)$ ground state.

\section{The metastable antiprotonic helium atom as antiproton trap}

Our picture of the exotic atom as a microscopic antiproton trap is most valid for the metastable antiprotonic helium atom mentioned in Sec. IV.B.2. The literature on this rather special $n \approx 38$ exotic atom is now extensive enough for a review in itself, and we present here only a short description of its main features. Metastable $\bar{p}-\mathrm{He}^{+}\left(\equiv e^{-} \mathrm{He}^{++} \bar{p}\right)$ atoms constitute about $3 \%$ of all antiprotonic helium atoms formed when $\bar{p}$ are brought to rest in helium (in any physico-chemical state); they remain in existence for some $10^{9}$ (instead of the usual $10^{2}$ ) antiproton orbital periods. A level scheme is shown for $\bar{p}-{ }^{4} \mathrm{He}$ in Fig. 14. The metastable states are characterized by large-angular-momentum quantum numbers $\ell$ and have lifetimes of the order of microseconds (Iwasaki et al., 1991; Yamazaki et al., 1993). The metastability is brought about by three effects. First, radiative transitions are slow due to the small transition energies $\Delta E$ (typically $\approx 2 \mathrm{eV}$ ). The radiative rate for the $n=38$ $\rightarrow n=37$ transition between circular orbits in $\bar{p}-{ }^{4} \mathrm{He}$, for example, is only about $0.7 \mu \mathrm{s}^{-1}$ (Yamazaki and Ohtsuki, 1992; Shimamura, 1992). Second, Auger transitions have to have large $|\Delta \ell|, \geqslant 4$, to eject the electron from the $\bar{p}-\mathrm{He}^{++} e^{-}$system, and are therefore slow (Russell, 1969). Finally, the Stark effect is suppressed during collisions with $\mathrm{He}$ atoms, and hence so-called sliding transitions (i.e., those with $\Delta n=0, \Delta \ell<0$ ) to short-lived 
Auger-depopulated states (wavy lines in Fig. 14), are suppressed as well: the energy difference $\Delta E(n, \ell$ $\left.\rightarrow n, \ell^{\prime}\right)$ of the levels with the same $n$ is increased by the electric field of the remaining electron, thereby appreciably decreasing the transition probability,

$$
\Gamma_{\text {Stark }}\left(n, \ell \rightarrow n, \ell^{\prime}\right) \propto\left[\frac{\left\langle n, \ell|e \cdot| \vec{E}|| n, \ell^{\prime}\right\rangle}{\Delta E\left(n, \ell \rightarrow n, \ell^{\prime}\right)}\right]^{2}
$$

(Lamb and Retherford, 1950). Furthermore, Pauli blocking between the remaining electron in $\bar{p}$-He and the two electrons in the $\mathrm{He}$ atom prevents the exotic atom from approaching helium atoms in the target closely enough to induce sliding transitions to shortlived levels: the transition matrix element $\langle n, \ell| e$ $\cdot|\vec{E}|\left|n, \ell^{\prime}\right\rangle, \vec{E}=$ electric field strength, remains small.

The cascade of the antiproton down the ladder of metastable states towards the shorter-lived levels, which are depopulated by Auger transitions with $|\Delta| \ell \leqslant 3$ at rates around $100 \mu \mathrm{s}^{-1}$, proceeds predominantly along levels with $v=n-\ell-1=$ const, because radiative transitions with $\Delta n=\Delta \ell=-1$ are strongly favored over all others. After formation, the $\bar{p}$-He atom recoils with kinetic energies of some $\mathrm{eV}$ and is slowed down by elastic collisions with other He atoms. The mean energy after $k$ collisions is a constant fraction

$$
f=\left[\frac{1-(1-\epsilon)^{2}}{2 \epsilon}\right]^{k} \approx 0.5^{k}
$$

of the initial energy (Condon and Breit, 1936), with $\epsilon$ $=4 m M /(m+M)^{2}$; here $m$ and $M$ are the masses of $\bar{p}$-He and $\mathrm{He}$, respectively. About ten collisions will then suffice to thermalize the $\bar{p}$-He atom.

The lifetime of metastable $\bar{p}-\mathrm{He}$ in pure $\mathrm{He}$ gas is determined by the number of metastable states in the cascade, the mean lifetime of each state, and collisional quenching through Stark mixing with short-lived states (Korenman, 1979). Taking all these effects together and assuming binary collisions, we may write the $\bar{p}$ annihilation rate

$$
\lambda=\lambda_{0}+\rho_{\text {atom }} \cdot \sigma_{\text {Stark }}\langle v\rangle
$$

with $\rho_{\text {atom }}$ the He atomic density, $\sigma_{\text {Stark }}$ the cross section for Stark mixing, and $\langle v\rangle$ the mean velocity of $\bar{p}-\mathrm{He}$ relative to the helium atoms in the gas.

A recent LEAR experimental arrangement (intended to be reproduced at the AD) is shown in Fig. 15. Antiprotons of $200 \mathrm{MeV} / c$ are extracted in bunches of about 200 ns length with about $10^{8} \bar{p} /$ bunch. These are stopped in a $\mathrm{He}$ gas target cooled to temperatures of several kelvin. Their collective fate, coming from multiple $\bar{p}$ annihilations into charged pions, is signaled as an analog pulse some tens of $\mu s$ long in a lucite Čerenkov counter. In order not to overload the counter, the high voltage of the attached photomultiplier is switched off during the prompt-annihilation peak (which contains $\approx 97 \%$ of the annihilations). The analog signal from the photomultiplier is recorded with a digital oscilloscope. Transitions from metastable to Auger-depopulated states are resonantly induced by a laser-light pulse fired

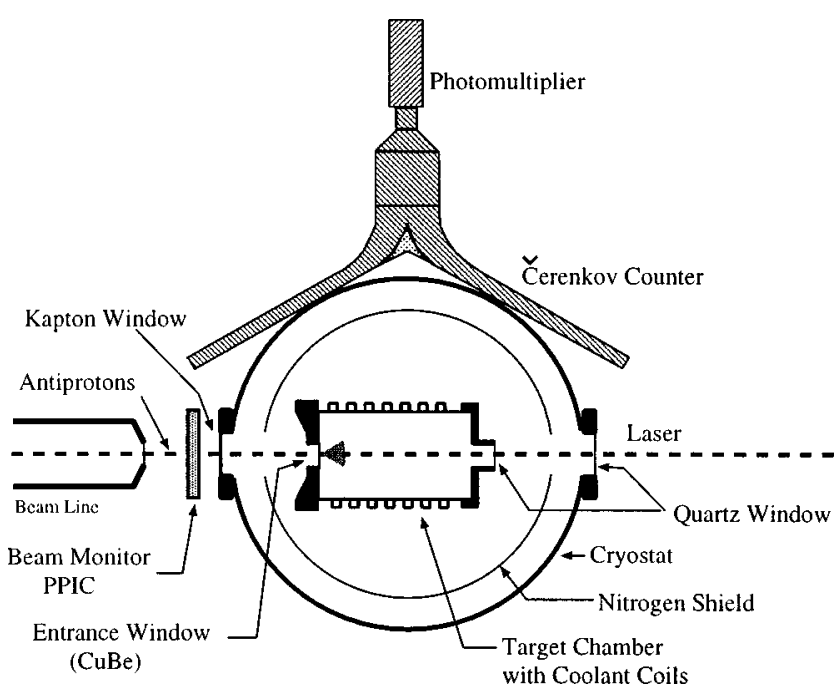

FIG. 15. Experimental arrangement for the laser-resonance studies on $\bar{p}$-He.

into the target through a quartz window at the downstream end. Further details may be found in Torii et al. (1997).

Our current knowledge of the metastable antiprotonic helium atom on the threshold of the $\mathrm{AD}$ era may be summarized as follows:

Atomic capture takes place preferentially into $\bar{p}$-He levels $n \geqslant 37$, as may be expected from the geometric similarity of the electron orbit before, and the antiproton orbit after, capture.

The observed value of $\sim 3 \%$ for the fraction of $\bar{p}$-He atoms that are metastable is compatible with an initial angular momentum distribution in the shape of a downward-bent parabola (Fig. 12), while the statistical $[p(\ell) \propto 2 \ell+1]$ initial distribution referred to in Sec. IV.B.2 gives a fraction about ten times higher.

Transition energies in $\bar{p}-{ }^{3} \mathrm{He}$ and $\bar{p}-{ }^{4} \mathrm{He}$ for, in all, 16 transitions, have been established by these laserresonance studies and are reproduced at the 5-ppm level by the most recent theoretical modeling of the threebody atom. It should be possible to use these existing results to determine an upper limit for the $\bar{p}-p$ mass difference comparable to that obtained from other $\bar{p}$-atom measurements; with the improved setup expected at the $\mathrm{AD}$, the comparison may be still further improved.

Quenching of the metastable states by collisions with other He atoms probably proceeds via Stark mixing of long- and short-lived states. The cross section for this process is at the $10^{-20} \mathrm{~cm}^{2}$ level, i.e., much smaller than the elastic-collision cross section.

One of the last results from LEAR was the resolution of the hyperfine structure of the $(n=37, \ell=35) \rightarrow(n$ $=38, \ell=34)$ transition in $\bar{p}^{4}$-He (Widmann et al., 1997). A precision measurement of the level splitting via microwave-induced transitions (to be discussed in Sec. VIII.B) may lead to an upper limit of the $\mu_{\bar{p}}-\mu_{p}$ difference that is meaningful as a test of $C P T$-symmetry.

This completes our discussion on holding antiprotons at rest and in isolation in microscopic and macroscopic 
traps. We now turn our attention to methods for doing the same thing with antihydrogen atoms.

\section{Confinement, cooling, and isolation of antihydrogen atoms}

Our arguments for the desirability of confining and isolating antiprotons in order to achieve the highest experimental precision on their properties are all the more true for the study of antihydrogen atoms, as can be seen from the recent rapid advances in ascertaining spectral transitions of ordinary hydrogen atoms, advances which have come largely from experiments on hydrogen atoms confined in neutral-atom traps or atomic beams. We present now a brief discussion of these devices; the larger problems of synthesizing antihydrogen atoms from their antiproton and positron constituents will be dealt with in Secs. VI.D, VII.B, and VIII.A.

\section{Neutral-atom traps}

Hydrogen and antihydrogen atoms are unaffected by the restraining forces developed by the electric and magnetic fields of Penning and Penning-Malmberg traps. They can, however, be confined within the potential well that an inhomogeneous magnetic field presents to the orbiting electron or positron. These possess permanent magnetic dipole moments $\mu\left(=5.79 \times 10^{-11} \mathrm{MeV} / \mathrm{T}\right)$ and will therefore have a potential energy $-\boldsymbol{\mu} \cdot \mathbf{B}$ in a field $\mathbf{B}$. As the nucleon moment is small compared with the electron or positron moment, this is close to the potential energy of the entire atom. If $\mu$ is antiparallel to $\mathbf{B}$, the atom will, in trying to minimize its energy, be drawn to magnetic-field minima; if it is parallel to $\mathbf{B}$, it will move away from these minima. Maxwell's equations forbid the existence of static magnetic-field maxima in free space so that low-field seekers will be trapped in the potential well around field minima while the high-field seekers will be ejected to the trap walls.

Neutral-atom traps based on this principle are by now well understood. A common design is that due to Pritchard (1983), in which the quadrupole field that confines the atoms radially is supplemented by a pair of coaxial solenoids at either end of the trapping region; these not only pinch the fields to give the axial inhomogeneity necessary for axial confinement but provide a nonzero bias field at the trap center, without which atoms in this region would undergo Majorana spin flips to high-fieldseeking states. The dipole force is weak; the well depth, $\mu \times \Delta B$, is measured in kelvin ( $\mathrm{meV}$ ) rather than $\mathrm{keV}$ for practically achievable field variations $\Delta B$ over the trap volume. Using the Ioffe-Pritchard design, Cesar et al. (1996) have achieved a precision of 2 parts in $10^{12}$ on measurements of the $1 S-2 S$ transition frequency in atomic hydrogen, and Setija et al. (1993) have made (less precise) measurements of the $1 S-2 P$ frequency.

Evidently positrons and antiprotons will have to be cooled to $\sim 1 \mathrm{~K}$ before recombination if the antihydrogen atoms formed are to stay inside a neutral particle trap built around the charged particle traps. This is still far too hot to permit the quantum limited (one part in
$10^{18}$ ) antihydrogen-hydrogen comparisons now under discussion. Supplementary neutral-atom cooling will therefore be required to reach the sub- $\sim \mathrm{mK}$ temperatures at which Doppler shifts (Hänsch and Zimmermann, 1993) have fallen to the same order of magnitude as the Zeeman and quantum limitations. The most promising technique for cooling hydrogen or antihydrogen to the $\mathrm{mK}$ level involves the scattering of Lyman- $\alpha$ laser light (Setija et al., 1993). Atoms moving against the laser beam will, owing to the Doppler shift, scatter its light with a slightly higher efficiency than those moving with it, with the net result that the approaching atoms slow down more than the receding ones speed up. The theoretical lower temperature limit for Lyman- $\alpha$ cooling of hydrogen, set by the isotropic recoil of the atom when it scatters the light, is $2.99 \mathrm{mK}$.

All the features discussed above appear in the design of Ioffe-Pritchard traps for AD experiments (Sec. VIII on antihydrogen).

\section{Atomic beams}

A beam of atoms with mean velocity $v$ and rms deviation $\Delta v$, diverging to a lateral size $d$ over a distance $L$ can be considered to be trapped within a cylindrical volume $\sim L d^{2}$ for a period $L / v$. Even at $10 \mathrm{~K}$ the speed of monoatomic hydrogen is some $400 \mathrm{~m} / \mathrm{s}$, resulting in a confinement time of only $\sim 0.4 \mathrm{~ms}$ in the 15 -cm-long beam used by Andreae et al. (1992) and Weitz et al. (1994) in their studies of the $1 S-2 S$ transition in atomic hydrogen. Although this is short compared with the 140-ms natural lifetime of the transition, these workers have recently (Udem et al., 1997) achieved an experimental precision of 3.4 parts in $10^{13}$ on the transition frequency, thus exceeding the precision cited above for the cold trapped atom technique.

The atomic beam therefore sacrifices the long storage times of the neutral-atom trap in favor of simplicity of construction and operation. To judge by the number of fundamental physical quantities that have been determined to high precision in the isolated atom environment found in atomic beams (Bayfield, 1977), this tradeoff has frequently been worthwhile. Examples include the fine-structure constant $\alpha$ (deduced from atomic-beam measurements on fine and hyperfine structures in one- and two-electron atoms) the proton magnetic moment, the Lamb shift, the equality of proton and electron charges (to better than one part in $10^{18}$ ), and upper limits on the electric quadrupole moment of the electron and proton.

The limitation of short confinement time when the beam velocity becomes too high is perhaps most evident in the antihydrogen synthesis experiments discussed in Sec. VI.D. In these experiments, the near-light-speed antihydrogen atoms were trapped in the above sense for a period of a few tens of nanoseconds only. As we shall see, ingenious quantum interference techniques can come to the aid of the experimenters even here for quite precise measurements of the antihydrogen Lamb shift. At the low-energy extreme (Sec. VII) experimental 
studies of eV beams of protonium $(\bar{p} p)$ and antiprotonic helium $\left(\bar{p}-\mathrm{He}^{++}\right)$ions are under careful consideration for the CERN AD program (ASACUSA, 1997), together with the possibility of applying atomic-beam techniques to antihydrogen studies.

During the early 1990s it was realized that, largely because of the technical developments described in previous pages, the antiproton had been placed in a new context - that of a laboratory for studying $C P T$ symmetry and the weak-equivalence principle at very high levels of precision. This laboratory began to function well before the end of the LEAR era and will be extended and refurbished by the advent of the AD. Before discussing these past and future studies of the antiproton (Secs. VI and VII), we must outline what are now the standard arguments used to justify such an ambitious undertaking.

\section{FUNDAMENTAL SYMMETRIES, ANTIPARTICLES, AND ANTIMATTER}

As the only stable antihadron, the antiproton should be rich in possibilities as a probe for fundamental symmetries. In vacuo its lifetime should, according to the $C P T$ theorem, be the same as that of the free isolated proton (presently known to be $>10^{32} \mathrm{y}$ for some modes), as should its mass and gyromagnetic ratio. By the same token, its additive quantum numbers (electric charge, lepton and baryon number) should have the same value but be opposite in sign to those of the proton. Bound in an exotic atom or a trap, it becomes a test body in an environment (the electromagnetic field) that in principle can be calculated to more or less arbitrary precision via QED; bound with a positron in an antihydrogen atom, it presents unequalled possibilities for high-precision spectroscopy.

\section{A. Antiprotons, antihydrogen, and the CPT theorem}

It is known that the existing experimental limit for CPT violation is very poor-the $10 \%$ level at best.

Kobayashi and Sanda (1992)

We defer discussion of this point of view to Sec. VII.D and summarize here some well-known background material concerning the $C P T$ theorem from Kabir (1965). The discontinuous operation $(C)$ relating free particle states to free antiparticle states is of course implicit even in the nonrelativistic, free-particle Schrödinger equation, where the complex conjugate of a solution is valid if it is taken to represent a particle of opposite charge and current density to the one represented by the original solution. Although it came into greater prominence with Dirac's attempt to find a Lorentz-covariant replacement for the Schrödinger equation, $C$ conjugation is not itself a Lorentz transformation. Furthermore, only proper Lorentz invariance ${ }^{13}$ is guaranteed when wave equations

\footnotetext{
${ }^{13}$ That is, invariance under velocity boosts, space rotations, space and time translations, and the identity.
}

are made Lorentz covariant. The improper transformations corresponding to space inversion alone $(P)$, time reversal $(T)$, and simultaneous inversion of both space and time, all clearly leave the interval invariant and therefore preserve Lorentz invariance but, like $C$, they cannot be obtained from any continuous sequence of infinitesimal transformations. The feature that no specific assumptions concerning $P$ and $T$ invariance need be made for proper Lorenz invariance (Hayward, 1990) is vitally important as it means that when the particles are allowed to interact, the interactions can break them both freely, as is indeed observed. And by implication, $C$ symmetry need not be assumed either.

We now try to understand particle physics (with considerable success) in terms of relativistic quantum field theories, not wave equations (Wess, 1988), but the arguments that $P$ and $T$ as well as $C$, when applied to freeparticle wave equation solutions, give equally valid alternative solutions, carry over into equivalent statements about free-particle (i.e., noninteracting) Hermitian fields (Kabir, 1965). The situation changes when we supplement free-particle relativistic quantum field theories with the minimal requirements necessary to take interactions into account, namely that interaction terms added to the Lagrangian density contain only the field operators and their derivatives (the locality condition) and that the usual spin-statistics relation holds. As noted in Sec. I.A, it turns out (Lüders, 1957) that, at least for scalar, spinor, and vector fields, these requirements make invariance of the theory under spacetime inversion unavoidable, without, however introducing new obligations concerning space or time inversion. ${ }^{14}$ Nevertheless, the time-inversion operation can only be given a consistent interpretation (e.g., Hayward, 1990) if it is associated with $C T$ instead of $T$ alone. ${ }^{15}$

The unavoidability of the connection between $C P T$ invariance and relativistic quantum field theories, even if the most general interactions between free fields are allowed, begs a number of questions:

Why, under these circumstances, are CPT tests of any interest at all?

Wess (1988) answers that in spite of the undoubted success of the standard model and QCD, the Higgs particle has not yet been seen, confinement is not fully understood, gauge invariances beyond the $S U(3)$ $\times S U(2) \times U(1)$ group are probably to be expected, and that, as discussed in the next section, gravitational relativistic quantum field theories seem impossible under the minimal axioms underlying the $C P T$ theorem. To this one might add that it is not known where the quarks

\footnotetext{
${ }^{14}$ Hayward also points out that, as spacetime has four dimensions, its inversion is equivalent to a finite complex proper transformation in which real rotations through angles $\pi$ are applied to two two-dimensional subspaces, maintaining reality of the coordinates only as a special case. This cannot be done for separate $C, P$, or $T$ operations.

${ }^{15}$ This distinction is emphasized by referring to $T$ alone as motion reversal, although time reversal continues to be used incorrectly.
} 
get their intrinsic masses; if they originate at the $10^{19}-\mathrm{GeV}$ Planck scale, the theoretical foundation of $C P T$ invariance appears to be unsound anyway (Lee, 1996). Moreover, the magnitude of the observed $C P$ violation is insufficient (Cohen, Kaplan, and Nelson, 1993) to explain the cosmic imbalance between matter and antimatter. It is really too early therefore to close the door on $C P T$-violating models.

At what level, then, would violations of CPT symmetry be important?

Here we must be careful not to give absolute significance to extremely small dimensionless numbers-tiny effects acting under cosmic conditions and for cosmic periods of time can change the look of the universe substantially. Thus weak interactions contribute "only" one part in $10^{7}$ to the Lagrangian, but the $P$ violation introduced by them has overwhelming consequences. One might then argue (questionably, according to Kobayashi and Sanda, 1992) that equality has nevertheless been established between the masses of the $\overline{K^{0}}$ and $K^{0}$ particleantiparticle pair to less than one part in $10^{18}$ (Carosi et al., 1990; Adler et al., 1997) and ask, is not a precision of one part in $10^{18}$ enough?

The answer must be a firm no for $C P T$ symmetrynot much bigger effects have extremely important consequences. For example, $C P$ violation in the neutralkaon system and the mass difference between long- and short-lived kaons $K_{L}$ and $K_{S}$ (both, at a few parts in $10^{15}$, equivalent to second-order weak-interaction effects, Barut, 1987) have extremely important consequences. Even electromagnetism produces a crucially important effect at the same $10^{-15}$ level-the hyperfine splitting of the hydrogen atom ground state. We might also recall that Einstein was prepared to introduce an imbalance of three parts in $10^{19}$ between the proton and the electron charges to explain the magnetic fields of the earth and the sun and that Bondi and Lyttleton (1959) tried to explain the expansion of the universe by the same mechanism (see also Petley, 1988). Finally, we might note that gravitational forces introduce terms of order only one part in $10^{40}$ into the interaction Lagrangian.

Then why antiprotons as probes of CPT invariance?

Because, as is evident from the discussions above and below, extremely-high-precision comparisons can be made between protons and antiprotons at rest and in isolation. Quantum limits apart, in cases where pairs of symmetry-related systems are available, the "degree of assurance" referred to by Park for a given symmetry is limited only limited by the precision of the experimental techniques. A good example is the comparison of the antiproton's $e / m$ value with that of the proton, obtained by measuring the cyclotron frequencies of these two species in a Penning trap (Gabrielse et al., 1995). This is a null experiment in which experimentally determined values for a system and its conjugate under the $C P T$ hypothesis of exactly equal values are compared. Even where null experiments are not possible (because, perhaps, the $C P T$ conjugate system is not available, as in the case of metastable antiprotonic helium), the degree of assurance may still be substantial, given the help provided by the powerful theoretical tools of QED.

And why antihydrogen atoms?

First, the ordinary hydrogen atom has for more than a century been the test bench at which we have refined and polished our understanding of the microscopic world; the antihydrogen atom should be just as useful a probe of the antiworld. Second, laser spectroscopic techniques should allow null comparisons of hydrogen and antihydrogen spectral frequencies with tremendously high accuracy, perhaps again as good as one part in $10^{18}$. By $C P T$ invariance, every detail of the atomic spectra of the two atoms should be the same; an example is the proposed comparison of the $1 S-2 S$ transition frequency in hydrogen and antihydrogen atoms, discussed in some detail in Secs. VII.C and VIII.A. One might argue here that the doubly exotic muonium $(M u)$ and positronium $(P s)$ hydrogenlike systems are much more freely available for $C P T$ tests than antihydrogen. They are in fact largely at a disadvantage, because $P s$ has a short life against annihilation and is self-conjugate, while $M u$, though not self-conjugate, is not available in the conjugate antimuonium $\overline{M u}=\mu^{-} e^{+}$form. Protonium ( $p$ $-\bar{p}$ ) is not only self-conjugate, it is subject to rapid annihilation unless (Sec. VIII.B) high- $n$ states can be observed in vacuo.

In fact the very success of $C P T$ symmetry seems to bring us to a paradoxical situation. Everyone admits that the standard model has many problems, and various attempts (e.g., Ellis, Mavromatos, and Nanopoulos, 1992; Colladay and Kostelecký, 1997) have been made to find a way out of these by abandoning one or more of the axioms. Yet no-one can point to a $C P T$-violating model as a serious rival to $C P T$-respecting ones and say that this or that difference should be looked for in that property of the proton and antiproton, because abandoning ingredients of the theorem in order to permit that difference means losing the hard-won gains of relativistic quantum field theories.

\section{B. Antiprotons, antihydrogen, and the weak-equivalence principle}

Aristotle claims that an iron ball of one hundred pounds, falling from a height of one hundred cubits reaches the ground before a one-pound ball has fallen a single cubit. I say that they arrive at the same time. You find, on making the experiment, that the larger precedes the smaller by two finger-breadths... Now you would not conceal behind these two fingers the ninety-nine cubits of Aristotle.

Galileo (1638)

When discussing the gravitational properties of antimatter, we are sooner or later forced to address a similar series of questions to the ones we met in connection with invariance under $C P T$ :

Does not the CPT theorem specify how antiparticles behave under gravity?

No. CPT invariance will hold for a given dynamical system if its Hamiltonian is such that the system's state 
vector $|\psi\rangle$ transforms into a new one $C P T|\psi\rangle$ which develops in time the same way as $|\psi\rangle$ does. If $|\psi\rangle$ represents, say, a system consisting of an antiproton falling to earth under gravity, the conjugate system is not an antiproton falling under the earth's gravity but an antiproton falling to an antiearth under its gravity. How antiprotons fall to earth is not constrained by $C P T$.

Would Galileo then be right for 1-kg iron and anti-iron balls (or $1 \mathrm{~kg}$ of protons and antiprotons)?

Not necessarily. We understand Galileo's (and later) results by recognizing that the coupling strength of a body to a gravitational field has nothing to do a priori with the normal concept of mass as the body's inertia, or resistance to acceleration produced by any means. The coupling strength is closer to the concept of charge familiar from the case of the electric field. Writing this gravitational charge or weight of an object $i$ as $Q_{i}$, the gravitational charge and radius of the earth and the acceleration produced by it at its surface as $Q_{E}, R_{E}$, and $g$, and the object's mass as $m_{i}$, Newton's second law gives

$$
G Q_{E} Q_{i} / R_{E}^{2}=m_{i} g .
$$

Galileo's result was therefore

$$
\begin{aligned}
Q_{i} / m_{i} & =g \times\left(R_{E}^{2} / Q_{E} G\right) \\
& =\text { const (independent of } i) .
\end{aligned}
$$

The gravitational charge/mass ratio was thus found to be the same for objects of the same composition but different sizes, when acted on by the earth's gravitational field. The experiments of Eötvös, Pekár, and Fekete (1922) extended this empirical law to objects of differing composition, and it became known as the principle of universality of free fall. Later still, Einstein formulated the law in terms of the weak-equivalence principle (WEP), making it the basis of the general theory of relativity, and explaining at last the mystery of the invariability of the gravitational charge-to-mass ratio by identifying the gravitational charge of bodies with their energy content $m c^{2}$, or more precisely, with their energy-momentum tensor. The most recent experiments of the Eötvös type (Adelberger et al., 1991) have shown that any WEP violation cannot exceed one part in $10^{11}$ in bulk matter.

To summarize, while the $C P T$ theorem must be respected by any quantum field theory that is consistent with special relativity, it is general relativity that must enter when making statements about the relative weights or gravitational couplings, of proton and antiproton. To the extent that the masses of particles and antiparticles are the same, their energy content is the same, and so of course should be their gravitational properties. Galileo would then be right about anti-iron balls if he assumed the weak-equivalence principle for antimatter, but he would still have to demonstrate it empirically. Although several general arguments exist on behalf of normal gravitational properties for antimatter, there are strictly no empirical data showing that it gravitates normally. ${ }^{16}$ Furthermore, as pointed out in critical discussions by Nieto and Goldman (1991), Hughes (1993a, 1993b), Charlton et al. (1994), and others, these arguments often assume the weak-equivalence principle, which is precisely the principle that ought to be subject to empirical testing.

Is the CPT theorem respected anyway in the presence of gravitational fields?

First, only scalar, spinor, and vector, and not tensor fields are included in the general proof of the theorem. For the first three, we are able to start from classical models, quantize them, use perturbation theory, and out comes a renormalizable theory. When we try the same thing with a spin-2 field it turns out to be nonrenormalizable. Consequently (Wess, 1989) gravity cannot yet be considered to derive from a quantum field theory. ${ }^{17} \mathrm{Sec}-$ ondly, Lorentz covariance is present among the axioms. The spin-2 gravitational field may or may not obey the CPT theorem, but Lorenz covariance is only locally valid in gravitational fields, where only the weaker condition of general coordinate invariance can be guaranteed. This may (Wald, 1980) be an obstacle to proving the theorem for general relativity.

As in the CPT case there is a paradox here, but it is of a different kind: studies of antiprotons and antihydrogen atoms at the extremely low energies needed to allow gravitational effects to be observed may allow us to put questions to Nature that she normally requires us to couch in extremely high-energy terms. Where, for example, has all the antimatter gone that supposedly constituted half the universe at its birth? The inadequacy of presently observed $C P$-violation effects to explain this, together with the absence of any empirical knowledge on the gravitational properties of antimatter, make it important to test the null hypothesis of equal gravitational couplings for matter and antimatter experimentally. Even a null result might be as significant for quantum gravity as the null result of Michelson and Morley was for special relativity, and that of Eötvös and Dicke for general relativity (Shiekh, 1997). A positive result would be sensational.

\section{STUDIES OF ANTIPROTONS AND ANTIHYDROGEN ATOMS TO DECEMBER 1996}

We now return to the experimental domain and evaluate past (this section) and future (Sec. VII) experi-

\footnotetext{
${ }^{16}$ Or, indeed, abnormally. Suspicions in the 1980s that there might be additional scalar and/or vector gravitational forces coupling to baryon or lepton number, which would have given protons and antiprotons slightly different gravitational properties, were laid to rest by Ericson and Richter (1990), who established an upper limit for such couplings of $10^{-6}-10^{-7}$ of the conventional (Einstein) one by inference from existing data for bulk matter, electrons, positrons, neutrons, and protons. See also Sec. VI.C.

${ }^{17}$ It is not at present known whether supergravity theories are renormalizable or not.
} 
mental studies of the antiproton and the antihydrogen atom in terms of the degree of assurance they furnish that Nature exhibits CPT symmetry and weakequivalence. It will quickly become apparent that it is only in the recent past that experiments have begun to provide any assurance at all. The reader might forgive us for digressing sometimes to discuss experiments with protons when these explain the shortfall in the antiproton case.

When we measure the properties of a given object, particulate or not, we are really making a comparison with the same property of a reference body or prototype. In an ideal experiment to check a symmetry principle the principle itself should define the choice of prototype, the measured quantity should depend on a single particle property predicted by the symmetry, and no assumptions other than the one being tested should be made in interpreting the result. An ideal $p-\bar{p}$ mass comparison to test $C P T$ symmetry would thus produce one number, depending only on mass, with the proton as the prototype, another number with the proton replaced in the apparatus by an antiproton, and a ratio of these two numbers whose difference from unity (if there is any) can be attributed to $C P T$ violation alone. Experiments that compare two systems under identical conditions are called null experiments.

Generally we can only approximate this ideal situation; thus the cyclotron frequency of trapped antiprotons [Eq. (7)] depends on the charge-to-mass ratio $q / m$, i.e., on two particle properties. Although the same frequency is measured with the proton as prototype in Gabrielse's series of experiments discussed in Sec. VI.B (Gabrielse et al., 1995), the ratio of these cannot be interpreted as a mass ratio without the (circular) assumption of $p-\bar{p}$ charge equality.

Still further from the ideal are measurements of antiproton transition frequencies in an exotic atom $\bar{p} A$; these can be thought of as measures of the Rydberg energy

$$
h c \cdot R y=m_{\bar{p}} q_{\bar{p}}^{2} \cdot q_{p}^{2} / 8 \epsilon_{0}^{2} h^{2} .
$$

Not only does $R y$ depend on $q_{\bar{p}}^{2} \cdot m_{\bar{p}}$ and not on $q_{\bar{p}}^{2}$ or $m_{\bar{p}}$, we do not even have the possibility of doing the same measurement on the $C P T$-conjugate atom $p \bar{A}$ as a prototype. As Hughes and Deutch (1992) have shown (cf. Sec. VI.B.3), it is possible to disentangle antiproton charge and mass measurements from cyclotron frequency and Rydberg measurements (see Secs. VI.B.1 and VI.B.2), but at considerable cost in precision.

Things get even more complicated in the case of the antiproton's magnetic moment. The fine-structure splitting is given in the Pauli approximation (Bethe and Salpeter, 1977) by

$$
\Delta E_{n, \ell} \approx\left(1+2 \kappa_{\bar{p}}\right)\left[\frac{\left(Z q_{\bar{p}} q_{p}\right)}{\hbar c}\right]^{4} \frac{1}{2 n^{3}} \frac{m_{\mathrm{red}} c^{2}}{\ell(\ell+1)},
$$

with

$$
\mu_{\bar{p}}=-\left(1+\kappa_{\bar{p}}\right) \cdot \mu_{\mathrm{N}} .
$$

This formula, from which the $\bar{p}$ magnetic moment is usually extracted (cf. Sec. VI.B.4), contains not only $\mu_{\bar{p}}$ but also the mass and the charge of the antiproton. Up to now this has posed no problem for the determination of $\mu_{\bar{p}}$, as this quantity is determined with much lower accuracy than $m_{\bar{p}}$, and $q_{\bar{p}}$.

\section{A. Direct measurements}

\section{Direct measurements of mass}

By a "direct measurement" of a particle property we mean one in which a single measured quantity determines the property without external assumptions. The most direct determination of the mass of a particle would then be via the kinematics of its motion. At first glance it could be argued that the first estimate of the antiproton mass made by Chamberlain et al. (1995; Segrè, 1958) shortly after its detection was kinematic, as the value was derived from the $\bar{p}$ momentum, evaluated from the radius of curvature $\rho$ of the particle track in a magnetic field to be $1.19 \mathrm{GeV} / c$, and the particle's $\beta$ values were constrained with the help of a Cerenkov counter to values between $0.75<\beta<0.78$ (a variant of the method determined the particle's velocity from the particle range). However, as the antiproton charge enters the equation for the radius of curvature, the determination does not really qualify as a direct measurement. Furthermore, the accuracy of such methods must always be limited, as $\rho$ (and even more, the Cerenkov angle) can only be measured with comparatively large errors.

The second method used in the earliest days of antiproton studies was based on the length and ionization density of particle tracks in photographic emulsions. As might be expected, the accuracy of these mass determinations was somewhat disappointing, $m_{\bar{p}}=(1824$ $\pm 51) \cdot m_{e}$ (Chamberlain et al., 1957; Segrè, 1958). Unfortunately, no better direct mass measurement seems to have resulted from the integrated production of some $10^{15}$ antiprotons ( $\equiv 2 n g$ ) at CERN, Fermilab or elsewhere since 1955 .

\section{Direct measurement of lifetime}

Compared with the lower limit for the total (mode independent) lifetime of the proton $\left[\tau_{p} \geqslant 1.6 \times 10^{25} \mathrm{y}\right.$ (Particle Data Group, 1996)] the lower limit for the lifetime of the $\bar{p}$ from the direct observation of antiproton survival time is pathetically small. As noted in the introduction, this was first estimated at the Bevatron (Chamberlain et al., 1955) as the time the antiprotons needed to fly from their creation point to the point of annihilation $\left(\tau_{\bar{p}} \approx 10^{-7} \mathrm{~s}\right)$. The next twenty years brought little progress, and in 1978 the lower limit was still only $\tau_{\bar{p}}$ $=120 \mu \mathrm{s}$ (at 95\% confidence), a result deduced from the inspection of 161 odd-prong events with net charge -1 observed after exposing a hydrogen bubble chamber to $760-\mathrm{MeV} / c$ antiprotons (Ganguli et al., 1978). As can be readily imagined, a breakthrough came when antiprotons were first contained in storage rings, again in 1978, 
TABLE IX. $\tau_{\bar{p}} / \mathrm{Br}$ ratio values for various decay channels for $\bar{p}$ decay (Geer et al., 1994). From experiment T861 at Fermilab.

\begin{tabular}{cc}
\hline \hline Channel & $\begin{array}{c}\tau_{\bar{p}} / \text { Br limit } \\
(95 \% \text { C.L. }) \text { in years }\end{array}$ \\
\hline $\bar{p} \rightarrow e+\gamma$ & $>1348$ \\
$\bar{p} \rightarrow e+\pi^{0}$ & $>554$ \\
$\bar{p} \rightarrow e+\eta$ & $>171$ \\
$\bar{p} \rightarrow e+K_{\mathrm{S}}^{0}$ & $>29$ \\
$\bar{p} \rightarrow e+K_{\mathrm{L}}^{0}$ & $>9$ \\
\hline \hline
\end{tabular}

whereupon the limit was raised by a factor of nearly $10^{9}$ to $32 \mathrm{~h}$ (Bregman et al., 1978; unfortunately no confidence level is given for this number). Antiprotons of $2.1 \mathrm{GeV} / c$ were stored in the ICE storage ring at CERN (Caron et al., 1978) and cooled stochastically, after which the time evolution of the beam was observed. Still another boost came with the development of small traps for antiproton storage. With a Penning trap Gabrielse et al. (1990) succeeded in storing about 1000 antiprotons (at a temperature of $4 \mathrm{~K}$ ) for more than two months, establishing directly a lifetime of $\tau_{\bar{p}} \geqslant 3.4$ months (at an unknown confidence level). It may be expected that this limit will be further increased in the future, after the AD at CERN has started operation.

Direct observation of the time evolution of antiproton beams as a means of determining $\tau_{\bar{p}}$ is strongly limited by the lifetime of both the experimental setups and the experimenters themselves. Another method (needing some input from theory) is the observation, or rather nonobservation, of products from $\bar{p}$ decay. Here it is helpful that only reaction products lighter than the $\bar{p}$ may emerge, and the decay products have to obey some conservation rules, such as those for charge or spin. All this restricts the possible number of decay channels, although the number searched for in the case of protons (Particle Data Group, 1996) is still impressive.

The first experiment of the kind just described was performed at ICE. Bell et al. (1979) searched with highefficiency detectors for the products of the decay of $2.1 \mathrm{GeV} / c \bar{p}$ into $\pi^{0}+e^{-}$. The limit for the lifetime in hours was found to be $\tau_{\bar{p}} \geqslant 1700 \mathrm{Br}$ (at $90 \%$ confidence); with the branching ratio $\mathrm{Br} \approx 0.20$ (Nanopoulos, 1978) this results in $\tau_{\bar{p}} \geqslant 340 \mathrm{~h}$.

At Fermilab the APEX test experiment (T861; Geer et al., 1994) and the APEX experiment itself (E868; Streets, 1997) provided the hitherto largest $\tau_{\bar{p}} / \mathrm{Br}$ value limits for $\bar{p}$ decay. T381 used a 144-module shower counter to search for decay products from $\bar{p}$ circulating with $8.9 \mathrm{GeV} / c$ in the antiproton storage ring. The Lorentz boost of the decay products helped to attain a good detection efficiency. With an integrated number of $(1.280 \pm 0.013) \times 10^{8}$ antiproton years the limits given in Table IX were achieved, while an improved experimental arrangement yielded the $\tau_{\bar{p}} / \mathrm{Br}$ ratios given in Table X (Streets, 1997). When set against the present lower limit of $10^{32}$ years for the proton lifetime for many decay modes, the CPT theorem prediction of equal proton and antiproton lifetimes cannot be said to have yet been
TABLE X. $\tau_{\bar{p}} / \mathrm{Br}$ ratio values for various channels for $\bar{p}$ decay. From experiment E868 at FermiLab (Streets, 1997).

\begin{tabular}{lc}
\hline \hline Channel & $\tau_{\bar{p}} /$ Br limit $(90 \%$ C.L. $)$ \\
\hline $\bar{p} \rightarrow e \gamma$ & $>1.3 \times 10^{6} \mathrm{y}$ \\
$\bar{p} \rightarrow e \pi^{0}$ & $>2.6 \times 10^{5} \mathrm{y}$ \\
$\bar{p} \rightarrow \mu^{-} \gamma$ & $>4.2 \times 10^{4} \mathrm{y}$ \\
$\bar{p} \rightarrow \mu^{-} \pi^{0}$ & $>1.8 \times 10^{4} \mathrm{y}$ \\
\hline \hline
\end{tabular}

seriously tested by experiment.

\section{Direct measurement of charge-bulk matter method}

As with all the other quantities characterizing the antiproton (and suitable for $C P T$ tests), the electric charge of the proton is known tens of orders of magnitude better than that of the antiproton. The proton experiments are of three kinds:

(1) the electric deflection of atomic (molecular) beams (Hughes, 1957),

(2) the electroacoustic effect (Dylla and King, 1973), and

(3) the electric neutrality of matter (Marinelli and Morpurgo, 1984).

The most precise value for $\Delta=q_{p}-q_{e}$ was attained (Marinelli and Morpurgo, 1984) in experiments with a ferromagnetic levitation electrometer (Marinelli and Morpurgo, 1982). In this device small steel balls (0.2-0.3 $\mathrm{mm}$ diameter) are held in (unstable) equilibrium with the gravitational force in a strong magnetic field by a feedback mechanism and exposed to a homogeneous electric field that works in horizontal direction. Carefully evaluating all possible forces on the steel spheres besides the electrostatic one, the authors arrive at a charge difference of $\Delta=(0.8 \pm 0.8) \times 10^{-21} e$. A prerequisite for this value to be valid is the neutrality of the neutron, which has been established at high accuracy, $q_{n}=(-0.4 \pm 1.1) \times 10^{-21} e$ (Gähler et al., 1982; Baumann et al., 1988). Thoughts about an improved experiment are described by Baumann et al. (1989); no further attempts have, however, been made to perform such a new experiment (Gähler, 1997).

These methods seem out of the question for antiprotons, although an atomic-beam experiment has been suggested that has something in common with both (1) and (3) (Hughes, 1957; Deutch, 1992). A beam of antihydrogen atoms would be injected between the plates of a parallel-plate capacitor. With plates of length $\ell$ charged to produce an electric field $\vec{E}$, the atoms will experience an acceleration

$$
\vec{a}=m_{\vec{p}}^{-1} \cdot(\Delta q \cdot \vec{E}+\nabla(\vec{\mu} \cdot \vec{E})+\nabla(Q \cdot \nabla \vec{E} / 6)+\cdots),
$$

where $\vec{\mu}$ is the magnetic and $Q$ the quadrupole moment of the $\overline{\mathrm{H}}$ atom. As was already pointed out by Hughes (1957), reversing the direction of the electric field would allow one to determine-and eliminate-the influence of the electric-field gradient at the edges of the parallelplate arrangement on the-polarizable-antihydrogen atom. The third term in Eq. (22) is small. The deflection of the beam is given by (Deutch, 1992) 


$$
\Delta y=y(\text { field up })-y(\text { field down })=\frac{\Delta q \cdot \ell^{2}}{2|\vec{E}| T_{\bar{H}}} .
$$

A (detectable) deflection of $\Delta y=25 \mu \mathrm{m}$ would follow for $\Delta q=10^{-8} e, E=100 \mathrm{kV} / \mathrm{cm}, T_{\bar{H}}=10 \mathrm{eV}, \ell_{2}=10 \mathrm{~cm}$. Although the experiment would be far from easy, such a limit would seem to be in the realm of the possible with some $10^{3}-10^{4}$ antihydrogen atoms, not far from the number currently being aimed at in projects at CERN and Fermilab.

\section{Direct measurement of the magnetic moment in traps}

The spin magnetic moment of the proton is one of the most precisely measured quantities in physics. By observing at the same time an electronic and a nuclear magnetic transition in atomic hydrogen, Winkler et al. (1972) succeeded in determining the electron-proton $g$-factor ratio, $g_{j}(\mathrm{H}) / g_{p}(\mathrm{H})=\mu_{j}(\mathrm{H}) / \mu_{p}(\mathrm{H})$ with $10 \mathrm{ppb}$ accuracy. From this the proton magnetic moment was derived to be $\mu_{p} / \mu_{B}=1.521032181(15) \times 10^{-3}$, again at an accuracy of $10 \mathrm{ppb}$. Quint and Gabrielse (1993) proposed to measure the $\bar{p}$ magnetic moment by trapping antiprotons in a 4-K Penning trap at 6 tesla. At this temperature a quantum-mechanical treatment of the $\bar{p}$ in the combined electric and magnetic fields is necessary. The resulting energy levels are characterized by $\hbar \omega_{\mathrm{c}}^{\prime}$, with $\omega_{\mathrm{c}}^{\prime}$ the eigenfrequency of the motion of the $\bar{p}$ in the electrostatic harmonic-oscillator potential, slightly modified by the axial magnetic field. Each oscillator level is split by the interaction of the antiproton magnetic moment $\mu_{\bar{p}}=1 / 2 \cdot g_{s} \cdot \vec{s}$ with the magnetic field. The anomaly frequency $\omega_{\mathrm{a}}$ is just the frequency of a transition that increases the oscillator quantum number by one unit and flips the antiproton spin from the higher to the lower energetic state (Brown and Gabrielse, 1986). Quint and Gabrielse (1993) wrote in a short note that they intended to excite the cyclotron motion in two steps via the reversed spin state by driving the spin flip as well as the anomaly transition of the antiproton by a magnetic dipole field at $250 \mathrm{MHz}$ and a magnetic quadrupole field at $160 \mathrm{MHz}$, respectively. They predicted that this method would yield the $\bar{p}$ magnetic moment with a relative accuracy of $10^{-9}$, which is considerably better than the best present-day value (Sec. VI.B.4). This interesting idea has not yet been experimentally verified because of the closure of LEAR, but once again, the CERN AD may provide the opportunity for such a confrontation with the $C P T$ theorem in the future.

\section{B. Indirect measurements}

One can only conclude from the previous section that precise, direct measurements of anything whatever concerning the antiproton are meager indeed. We therefore turn to indirect experimental methods for determining this particle's mass, lifetime, charge, and magnetic moment, either by measuring values representing a combination of these quantities or by relying heavily on theoretical assumptions.

\section{Cyclotron frequencies and mass/charge determinations}

The first attempt at determining $q / m$ in the era of the modern low-energy antiproton machines employed the Orsay rf mass spectrometer (de Saint Simon et al., 1995). A spectrometer originally built at Orsay in order to measure atomic masses with high precision was adapted to compare-at LEAR - the charge-to-mass ratio of $\mathrm{H}^{-}$ ions and $\bar{p}$ with an accuracy of $10^{-9}$. This required a mass resolving power of $10^{6}$, which was achieved, and an intense, low-emittance beam of $200-\mathrm{keV}$ antiprotons, which, as recognized by the authors, was not.

Beginning in 1990, Gabrielse et al. (1990) succeeded, with a Penning trap some $10^{4}$ times smaller in linear dimensions, in comparing the cyclotron frequencies of $\sim 100$ protons and antiprotons stored at $4.2 \mathrm{~K}$ in the same magnetic field $\vec{B}$. The resulting ratio,

$$
R=\frac{(q / m)_{\bar{p}}}{(q / m)_{p}}=1-(0.2 \pm 0.4) \times 10^{-7},
$$

constituted the first really precise test of the CPT theorem for hadrons. Since 1990, the method has been refined and the relative errors reduced by a further factor of 40 , bringing the ratio to $R=1+(1.5 \pm 1.1) \times 10^{-9}$ (Gabrielse et al., 1995). We stress, however, that the equality of the charge-to-mass ratio on the ppb level does not prove mass equality at the same level, as the equality of proton and antiproton electric charge has only been established with considerably lower precision, either directly or (Hughes and Deutch, 1992) by including Rydberg energy determinations with the cyclotron frequency measurements as described in Sec. VI.B.3.

\section{Exotic atoms: The antiprotonic Rydberg and mass/charge determinations}

Well before the "antiproton factories" at CERN and FermiLab went into operation, the mass of the $\bar{p}$ had been inferred from the transition energies of antiprotonic atoms (Hu et al., 1975; Roberts et al., 1975; Roberson et al., 1977). In order to avoid complications from the finite nuclear size (Sec. IV.B.3), transitions between high-lying orbits were chosen. The nuclear charge outside the mean radius of the well localized $(n=12, \ell$ $=11$ ) orbit in antiprotonic lead is, for example, only $\approx 10^{-35}$ of the maximum density, and corrections remain very small. To keep the energy to be determined in a range easily accessible with solid-state detectors, a highatomic-number exotic atom had to be chosen. In fact, such measurements only became feasible after detectors with good energy resolution had been developed.

An experimental arrangement for a precision measurement of transition energies will have the following general features: Antiprotons will be detected with the help of a scintillation counter telescope and stopped in the target, with the number of stops maximized by an adjustable degrader. $\mathrm{X}$ rays from the target in prompt coincidence with a stop signal from the telescope will be registered, together with $\gamma$ rays from a calibration source positioned near the target. This will minimize the errors 
TABLE XI. Experimental results for mass and magnetic moment of the antiproton as derived from solid-state detector experiments. A reevaluation by Pilkuhn and Schlaile (1983) decreased the value for the $\bar{p}$ magnetic moment to $\mu_{\bar{p}}$ $=-2.794(48) \cdot \mu_{\mathrm{N}}$.

\begin{tabular}{lcc}
\hline \hline \multicolumn{1}{c}{ Reference } & $\bar{p}$ mass $\left(\mathrm{MeV} / c^{2}\right)$ & $\begin{array}{c}\bar{p} \text { magnetic } \\
\text { moment }\left(\mu_{\mathrm{N}}\right)\end{array}$ \\
\hline Hu et al. $(1975)$ & $938.179(58)$ & $-2.791(21)$ \\
Roberson et al. $(1977)$ & $938.229(49)$ & \\
Roberts (1978) & $938.13(13)$ & $-2.817(48)^{*}$ \\
Average & $938.202(36)$ & $-2.795(19)$ \\
\hline \hline
\end{tabular}

in the energy calibration. Low background and good energy resolution will be essential and a strong exotic x-ray line has to be chosen.

In order to extract values for the $\bar{p}$ mass, the measured transition energies have to be compared to those calculated by solving the Dirac equation (cf. Sec. IV.B.3), applying all necessary corrections and adjusting the $\bar{p}$ mass to obtain optimum agreement.

Table XI, second column, shows the results for the antiproton mass from the three experiments already mentioned and their weighted average. This average, 938.202(36) $\mathrm{MeV} / c^{2}$, is in reasonable agreement with the value for the proton mass, $m_{p}$ $=938.27231(28) \mathrm{MeV} / c^{2}$ (Particle Data Group, 1996), but with quite a large relative error of $40 \mathrm{ppm}$.

Taking into account that nowadays $\pi^{-}$mass measurements with crystal spectrometers reach $2.5 \mathrm{ppm}$ accuracy (or better), a remeasurement of the $\bar{p}$ mass with a crystal spectrometer would be very desirable.

The very precise measurement of transition energies between metastable levels in $\bar{p}$-He (cf. Sec. IV.B.5) has recently permitted somewhat better conclusions on the antiproton mass, although indirect to the extent that they are inferred from its Rydberg constant. Eight transitions with $\Delta v=0$ have now been measured for $\bar{p}-{ }^{4} \mathrm{He}$ in all cascades from $v=0$ to $v=3$. For these transitions many of the uncertainties in the level energies cancel as energy differences for $\Delta n=-1$ are determined. The maximum deviation for all these measured energies from calculations (Korobov, 1996) is smaller than $5 \mathrm{ppm}$ and the mean deviation is only $3 \mathrm{ppm}$, assuming for $\bar{p}$ mass and charge, respectively, the value of proton mass and charge. We can conclude that the antiprotonic and protonic Rydberg values are equal at the 3-ppm level of accuracy.

\section{Exotic atoms and the antiproton charge}

We have already mentioned in the introduction to this section that by combining cyclotron frequency and Rydberg energy measurements, either the antiproton charge or its mass can be determined indirectly. Hughes and Deutch (1992) combined the cyclotron frequency ratio

$$
\omega_{p \bar{p}} \equiv \frac{\omega_{\bar{p}}}{\omega_{p}}=\frac{q_{\bar{p}} \cdot m_{p}}{q_{p} \cdot m_{\bar{p}}}
$$

with the Rydberg energy of the antiprotonic hydrogen atom normalized to that of the electronic hydrogen atom

$$
R y_{p \bar{p}}=\frac{2 m_{\mathrm{red}}}{m_{e}} \frac{q_{\bar{p}}^{2} \cdot q_{p}^{2}}{e^{4}}
$$

to extract the antiproton/electron charge ratio. Taking these formulas and using the latest values $\omega_{p \bar{p}}=1+(1.5$ $\pm 1.1) \times 10^{-9}$ and $R y_{p \bar{p}}=1-(7 \pm 4) \times 10^{-5}$ (Table XI), we may derive $q_{\bar{p}} / e=1 \pm 1.6 \times 10^{-5}$. The comparatively large error is entirely governed by the restricted precision of the $\bar{p}$-atom experiments on the $\bar{p}$ mass (Sec. VI.B.2).

\section{Magnetic moment from exotic atoms}

As we saw already in the introduction to this section, fine-structure splitting of high-lying $(n, \ell)$ levels in heavy antiprotonic atoms can be used to determine the $\bar{p}$ magnetic moment $\mu_{\bar{p}}$. For these levels corrections of the binding energy are small and mostly cancel for the energy difference between the levels (cf. Table VIII). The splitting $\Delta E_{n, \ell}$ is, for a point nucleus, given by Eq. (21). Including the recoil effect, which increases the contribution of the Pauli term to the fine-structure splitting, leads to (Bohnert et al., 1986)

$$
\begin{aligned}
\Delta E_{n, \ell}= & {\left[1-m_{\bar{p}}^{2} /\left(m_{\bar{p}}+m_{N}\right)^{2}+2 \cdot \kappa_{\bar{p}} m_{N} /\left(m_{\bar{p}}+m_{N}\right)\right] } \\
& \times\left[\frac{\left(Z q_{\bar{p}} q_{p}\right)}{\hbar c}\right]^{4} \frac{1}{2 n^{3}} \frac{m_{\mathrm{red}} c^{2}}{\ell(\ell+1)} .
\end{aligned}
$$

The only viable way, however, is to integrate the Dirac equation for a finite-size nuclear potential including the Pauli term for the anomalous magnetic moment and a vacuum polarization (VP) potential representing the first two orders of the VP corrections. All other corrections to the binding energy may be treated as perturbations (Borie, 1983). Comparing the fine-structure splitting calculated on the basis of the contribution of $\mu_{\bar{p}}$, one may draw conclusions on the deviation of $\kappa_{\bar{p}}$ from the canonical value.

The first such measurement was performed by Roberts et al. (1975) using antiprotonic x rays from lead and uranium. The experimental setup for such $\mathrm{x}$ ray experiments was described in Sec. VI.B.2. The weighted average from six lines (three from $\bar{p}-\mathrm{Pb}$, three from $\bar{p}$ - $\mathrm{U}$ ) came out to be $\mu_{\bar{p}}=(-2.819 \pm 0.056) \mu_{\mathrm{N}}$. A reevaluation by Roberts (1978), taking the advanced knowledge on corrections into account, gave $\mu_{\bar{p}}=(-2.817 \pm 0.048) \mu_{\mathrm{N}}$ (Table XI), about $0.1 \%$ lower than the previous value of these authors and in agreement with the $C P T$ prediction. At about the same time $\mathrm{Hu}$ et al. (1975), exploiting the fine-structure splitting of the $(11 \rightarrow 10)$ and $(12$ $\rightarrow 11)$ transitions in antiprotonic lead and uranium, arrived at $\mu_{\bar{p}}=(-2.791 \pm 0.021) \mu_{\mathrm{N}}$.

Improved antiproton beams and Ge-detector equipment enabled Kreissl et al. (1988) to obtain, with only two lines from antiprotonic lead, the current best antiprotonic atom value, $\mu_{\bar{p}}=(-2.801 \pm 0.009) \mu_{\mathrm{N}}$. This 


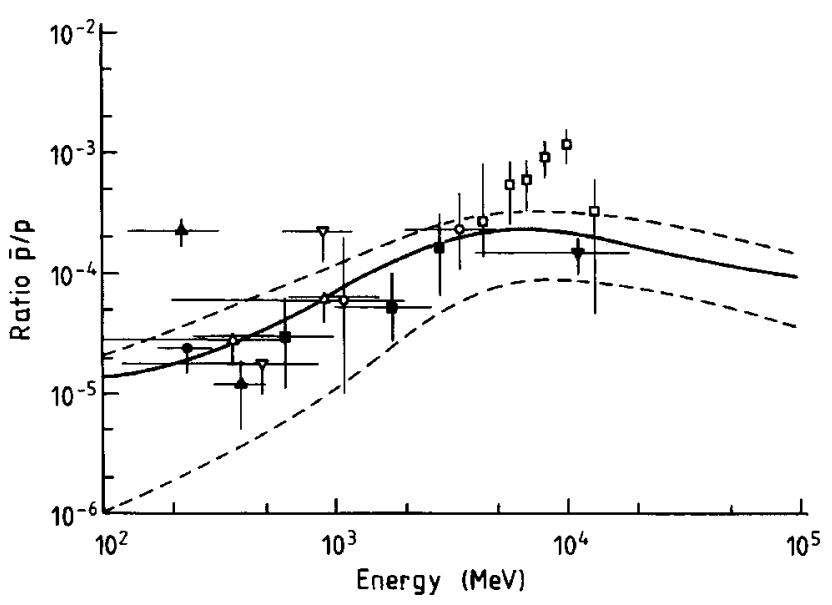

FIG. 16. Results for the cosmic-ray flux of antiprotons and protons: $\square$, Golden et al., 1979; ○, Bogomolov et al., 1990; $\triangle$, Ahlen et al., 1988; $\nabla$, Streitmatter et al., 1989; $\mathbf{0}$, Mitchell et al., 1996; ○, Orito et al., 1995; $\mathbf{\Delta}$, Buffington et al., 1981; $\mathbf{\nabla}$, Hof et al., 1996.

means a relative difference between the magnetic moments of proton and antiproton of

$$
\frac{\mu_{p}-\left|\mu_{\bar{p}}\right|}{\mu_{p}}=(-2.4 \pm 2.9) \times 10^{-3}
$$

Although this value is compatible with zero, it cannot really be said to constitute a significant test of the $C P T$ theorem.

\section{Lifetime from the antiproton abundance in cosmic radiation}

As already indicated, the limits on the $\bar{p}$ lifetime from direct measurements and from the observation of the decay products are still quite low. Rather early in the antiproton's history, astrophysics was able to set a much more stringent lower limit if certain cosmological assumptions were made. From the fact that the measured antiproton flux in the upper atmosphere is compatible with the assumption that the $\bar{p}$ are produced in secondary reactions with cosmic rays, Golden et al. (1979) concluded that the lifetime of the $\bar{p}$ has to be $\tau_{p} \geqslant 1.6$ $\times 10^{7} \mathrm{y}$, the cosmic-ray storage time. A number of experiments have since then been performed (Ahlen et al., 1988; Bogomolov et al., 1990; Mitchell et al., 1996) to determine the antiproton flux as a function of energy. Their results are compiled in Fig. 16. The flux of cosmicray antiprotons is reasonably well reproduced by the two cosmological models shown in the figure (Webber and Potgieter, 1989; Gaisser and Schaefer, 1992). Hence it seems established that the $\bar{p}$ seen with energies below $3 \mathrm{GeV}$ have their origin in the reaction of cosmic-ray particles (mostly protons). As the flux of the antiprotons is not lower than expected, no antiprotons may have disappeared and their lifetime must be comparable to or greater than the storage lifetime of the cosmic rays generating them. This lifetime has been estimated from the abundance of ${ }^{10} \mathrm{Be}$ to be $\approx 10^{7}$ y (Garcia-Muñoz and Simpson, 1988).
An even better lower limit for $\tau_{\bar{p}}$ was derived by Stephens and Finetti (1996), who studied the diffuse radiation generated by the decay

$$
\begin{aligned}
\bar{p} \rightarrow e & (459.4 \mathrm{MeV} \text { kinetic energy) } \\
& +\pi^{0} \text { (478.8 MeV kinetic energy). }
\end{aligned}
$$

The inverse Compton effect of the electrons with photons of the blackbody radiation account for the lowenergy $(\approx 1 \mathrm{keV})$ part of the spectrum, whereas the same process with starlight produces photons in the $\mathrm{MeV}$ region. $\pi^{0}$ decay, finally, leads to two $\gamma$ rays with a flat energy distribution between 10 and $470 \mathrm{MeV}$ and a sharp high-energy cutoff. Comparing spectra calculated under the assumption of a symmetric universe and a $\bar{p}$ lifetime of $1.0 \mathrm{~s}$ (left-hand flux scale of Fig. 17) with those observed in different satellite experiments (righthand flux scale of Fig. 17), the authors find $\tau_{\bar{p}}>1$ $\times 10^{17} \mathrm{y}$, unfortunately without confidence level. This limit is seventeen orders of magnitude higher than the present-day limit from antiproton decay experiments on earth and sixteen orders of magnitude smaller than the $\tau$ value for $p \rightarrow e^{+}+\pi^{0}$ decay (Becker-Szendy et al., 1990).

\section{Antiproton ballistics}

The determination of the weight or gravitational acceleration of the bare antiproton, simple as the principle may be, is a very demanding challenge (Beverini et al., 1986a, 1986b; Goldman et al., 1987). The recipe is simple, and was also the basis of an attempt by Witteborn and Fairbank (1967) to measure the electron and positron weights ${ }^{18}$ : Launch low-energy antiprotons upwards and record the time they need to reach a given height (Holzscheiter, 1991). The longest time observed, $t_{\max }$, is taken by the particles just reaching the upper end of the path $h$, given by

$$
h=\frac{g}{2} t_{\max }^{2} \Rightarrow g=\frac{2 h}{t_{\max }^{2}}
$$

independent (according to the weak-equivalence principle) of the particle's mass. If we know $h$ and $t_{\max }$ we may derive the gravitational acceleration $g$ of the $\bar{p}$ in the earth's field. The extreme difficulties lie in the details. In order to reach reasonable values for $h$ (a few meters at most), $t_{\max }$ has to be of the order of $\mathrm{s}$ and the kinetic energy of the $\bar{p}, T=(m / 2) v^{2}=m \cdot g \cdot h$, has to be in the $\mu \mathrm{eV}$ region. This means that in order to get a sizable fraction of antiprotons of such extremely low energy, the $\bar{p}$ sample has to be cooled to kelvin temperatures. Another difficulty lies in the weakness of the gravitational force. To exert an electric force of the same

\footnotetext{
${ }^{18}$ The misunderstanding persists that this experiment showed that the gravitational accelerations of positrons and electrons are equal to $10 \%$. In fact the $g$ of the electron was shown to be within $10 \%$ of $9.81 \mathrm{~ms}^{-2}$, but no measured value was obtained for the positron, owing to the systematic effects mentioned below.
} 


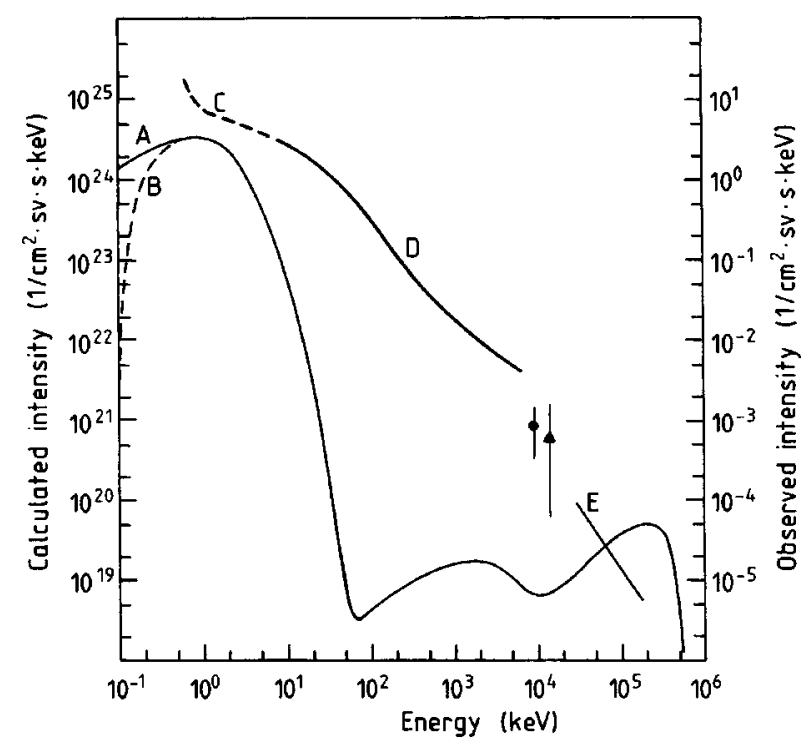

FIG. 17. Estimated intensity of the diffuse radiation in the intergalactic space $(\mathrm{A}, \mathrm{B})$ compared with the results of observations ( $\mathrm{C}-\mathrm{E}$ and full circles and triangles) (Stephens and Finetti, 1996). For further explanations see text.

size as the gravitational one on a proton (or antiproton), the electric field need only be $E \approx 10^{-7} \mathrm{~V} / \mathrm{m} .{ }^{19}$ The requisite field-free environment would normally be obtained by letting the charged particle fly inside a long cylindrical screening tube. In practice things are much less simple, and Darling et al. (1992) have given a detailed description of the residual electrical forces acting on the particle in such a tube. The most important effects are

(1) electrical forces by contact-potential patches (the patch effect; Herring and Nichols, 1949) by a shift of the electrons in the tube due to gravitational forces (Dessler et al., 1968), by the electric field leaking in at the end of the tube, and by a possible off-axis movement of the particle;

(2) forces due to magnetic fields which guide the $\bar{p}$ away from the tube axis;

(3) forces due to the interaction of the antiproton magnetic moment with magnetic stray fields;

(4) scattering on residual gas atoms.

These effects, together with the low yield of $\mu \mathrm{eV}$ antiprotons have up to now prevented this experiment from passing the design stage, while Ericson and Richter have questioned the usefulness of its $(1 \%-0.1 \%)$ projected precision (see footnote 16). Attention is, in any case, now mainly directed towards ballistic experiments with antihydrogen atoms (Holzscheiter et al., 1996). Here the neutrality of the projectile suppresses the most disturbing of the nongravitational interactions, the pure Coulomb interaction. Even the rather high polarizability of the antihydrogen atom leads only to a very weak force

\footnotetext{
${ }^{19}$ More graphically, the electric force on an antiproton from a proton or electron $10 \mathrm{~cm}$ distant is larger than the gravitational force between it and all the $4 \times 10^{51}$ neutrons and protons in the earth.
}

for reasonable field strengths and gradients. For this advantage one pays a price. The formation of low-energy antihydrogen is an up-to-now unsolved problem (Sec. VII.B); furthermore, the $\bar{p} e^{+}$system may no longer be easily guided by a magnetic field along a direction antiparallel to the gravitation field lines.

Recently a new ballistic method has been proposed to measure the gravitational acceleration (Lagomarsino et al., 1994; Lagomarsino et al., 1996): the gravityinduced shift of the center of the radial orbits of $\bar{p}$ in a Penning trap. $\mu \mathrm{eV}$ antiprotons are injected near the center of the trap (axial dimension $z_{0}$, voltage well depth in axial direction $V_{0}$ ), which is operated with the magnetic field perpendicular to the direction of the gravitational acceleration. The gravitation makes the guiding center of the cyclotron motion move on a circle with the center at $\left(x_{0}=m \cdot g \cdot z_{0}^{2} /\left(q \cdot V_{0}\right), y=0\right)$ and with a period $T_{\mathrm{m}}$ $=2 \pi / \omega_{\mathrm{m}}, \omega_{\mathrm{m}}=$ magnetron frequency. Here the $x$ axis is chosen parallel to the gravitational force. If the $\bar{p}$ were injected along the trap axis $(x=0)$ its position after $T_{\mathrm{m}} / 2$ would be just $2 \cdot x_{0}$. A measurement of this position allows us to determine $g$, as $q / m$ is known very well from the cyclotron-frequency measurement (see Sec. VI.B.1). The authors claim that electric forces on the $\bar{p}$ due to the patch effect, which are ten times stronger than the gravitational force, may still be tolerable. As with the other ballistic experiments, there is not yet even an experimental proof of principle.

\section{Experiments with "hot" antihydrogen}

We have discussed at some length the technical problems of reducing the energy of antiprotons to the thermal or subthermal regime and the (limited) extent to which high-precision comparisons of their various properties with those of protons have been interpretable as tests of the CPT theorem. The recombination of these ultralow-energy antiprotons with positrons to form antihydrogen has, with the advent of the AD machine, become one of the main reasons for achieving these conditions. However, two experiments, one at Fermilab and the other at CERN, have recently succeeded in synthesizing antihydrogen atoms at $\mathrm{GeV}$ energies. We now digress from our main theme to describe these experiments and evaluate their potential critically, before returning to a discussion of antihydrogen synthesis at extremely low energies. A more detailed discussion can be found in the review of Greenland (1997).

The successful searches for antihydrogen atom production at $\mathrm{GeV}$ energies (Baur et al., 1996, Christian et al., 1997) followed a 1992 suggestion made by Munger et al. (1993) and further developed by Munger, Brodsky, and Schmidt in 1994. The production mechanism involves the creation of electron-positron pairs during the passage of an antiproton through the Coulomb field of a nucleus $(Z, A)$. Occasionally the positron will remain bound to the antiproton, and owing to the extremely small momentum transferred, the resulting antihydrogen atom will emerge on almost exactly the original antiproton trajectory. 
This process is of course purely quantum electrodynamic, with a cross section estimated by Munger et al. (1993) as

$$
\sigma\left[\bar{p}+(Z, A) \rightarrow \bar{H}+e^{-}+(Z, A)\right] \sim 4 Z^{2} \mathrm{pb}
$$

for antiproton momenta above $\sim 6 \mathrm{GeV}$.

Although of a high order in $\alpha$, this can be conceptually understood in terms of $e^{+} e^{-}$annihilation, the timereversed equivalent of

$$
\gamma+\gamma \rightarrow e^{+}+e^{-}
$$

occurring via the interaction of virtual photons as the antiproton and $Z$ atom pass each other. The estimated cross section (Bertulani and Bauer, 1988) is

$$
\sigma \sim\left(Z r_{B}\right)^{2} \alpha^{6} \times \ln (\gamma),
$$

where $\gamma$ is the kinematic factor $E / M$ and $r_{B}$ the $1 S$ Bohr radius of the electron in hydrogen. Taking account of the further requirement that the positron must not only be created but captured into a $1 S$ state introduces an extra factor of $\alpha^{3}$ and gives the partial cross section

$$
\sigma_{1 S} \sim Z^{2} r_{B}^{2} \alpha^{9} \sim Z^{2} \ln (\gamma) \mathrm{pb}
$$

for producing ground-state antihydrogen.

The experiment reported in Baur et al. (1996) was done at LEAR (Fig. 5) using a xenon $(Z=54)$ jet target. The xenon atoms in the jet crossed the path of the circulating $1.94-\mathrm{GeV} / c(\beta=0.90, \gamma=2.3)$ antiproton beam, which traversed the jet some $4 \times 10^{6}$ times per second. The presence of the jet reduced the beam lifetime from several hours to about three minutes; its intensity was increased from $1 \times 10^{12}$ to $3 \times 10^{13}$ atoms s${ }^{-1}$ during the twelve-minute runs in order to maintain an approximately constant luminosity or event rate. Once formed, the $\sim 1.96-\mathrm{GeV} / c \bar{H}$ atoms were no longer confined in the LEAR ring and left it (Fig. 5) at the next bend, some $10 \mathrm{~m}$ from the jet. To confirm that the neutral particle was indeed an antihydrogen atom and not a neutron, the positron was stripped in the first of a series of three thin silicon counters just before the exit window but outside the bending magnet field. The resulting equal-velocity $e^{+}$and $\bar{p}$ had kinetic energies $0.663 \mathrm{MeV}$ and 1217 $\mathrm{MeV}$, so that the positron stopped in the silicon array and annihilated into two 511-keV photons, detected in a $\mathrm{NaI}$ crystal, while the antiproton continued through a dipole magnet and itself annihilated in a downstream array of scintillation counters. Candidate disassociated antiprotons were selected by a combination of constraints on the time of flight, pulse height, and deflection in the dipole, correlated with the information from the silicon array. During 15 hours of beamtime, eleven events were observed which survived all selection procedures on antiprotons and electrons and could therefore be deemed to be antihydrogen events. An estimate of background nevertheless gave an expected count of two during the 15 -hour run period. These figures are in rough agreement with what is expected from Eq. (26).

The experiment carried out in the Fermilab Antiproton Accumulator by Blanford et al. [1998a; see also Christian et al. (1997), Fig. 4, and Fig. 18] is very similar

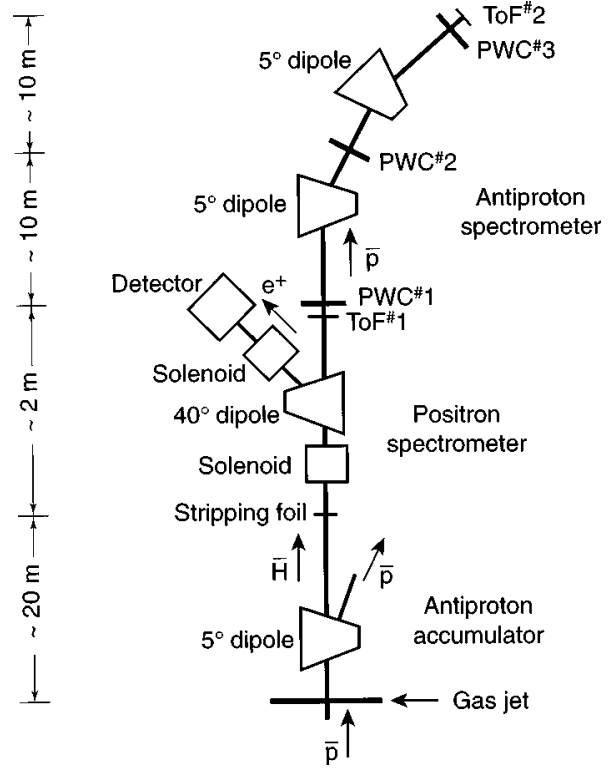

FIG. 18. Layout of the FERMILAB antihydrogen experiment, after an original diagram supplied by M. Mandelkern. See Fig. 4.

in layout and conception, although a hydrogen jet took the place of a xenon one, the $\gamma$ factor was considerably higher ( $\sim 9.5$ for $P=8.9 \mathrm{GeV} / c$ ), and the flight paths of both the antihydrogen atom and the disassociated antiproton were somewhat longer $(24 \mathrm{~m}$ and $26 \mathrm{~m}$, respectively). In addition, the positron was momentum analyzed by a separate dipole magnet before it annihilated. To date 57 events have been recorded.

The LEAR experiment was not undertaken with the aim of measuring any property of the antihydrogen atom, but Greenland has deduced a limit on its net charge from the fact that the atoms did in fact emerge from the LEAR bending magnet. The geometrical dimension of the exit port constrained the gyromagnetic radius $R_{\bar{H}}$ of $\bar{H}$ in the LEAR ring relative to that of the $\bar{p}$, and this in turn constrained the net $e^{+} \bar{p}$ charge difference to the value

$$
|\delta q / e| \leqslant \rho_{\bar{p}} / \rho_{\bar{H}}=1.9 \times 10^{-2} .
$$

This is far from being even as stringent a constraint on the positron-antiproton charge difference as the value of one part in $10^{5}$ deduced by Hughes and Deutch (1992) and referred to in the introductory paragraph to this section, but it can be seen as a primitive measurement of the net charge of bulk antimatter as suggested by Deutch (1992). The closure of LEAR has effectively put an end to such experiments at CERN, but the Fermilab experiment will continue for some time with attempts to measure the $n=2$ ionization cross section, the finestructure constant, and the Lamb shift for antihydrogen.

These experiments, suggested by Munger et al. (1994), and now proposed by Mandelkern et al. (1997) and Blanford et al. (1998b) are null CPT tests (albeit at low precision) to the extent that they can be repeated with hydrogen atoms recombined from protons in the same apparatus. We refer to them by the Fermilab proposal 
number E862. In the ionization experiment the 8.9$\mathrm{GeV} / c$ atoms (now emerging from a xenon jet) will be excited with an efficiency of about $10 \%$ to the $n=2$ manifold by passing them through a thin membrane located in a 0.7-T magnetic dipole field. The $\mathbf{v} \times \mathbf{B}$ motional electric field will ionize these $S$ - and $P$-state atoms with a state-dependent time constant and produce a new source of antiprotons distributed along the magnet axis over a distance $\sim 10 \mathrm{~cm} .{ }^{20}$ The mean lateral deflection of these when they annihilate on a downstream plane can be measured relative to the annihilation point of the (undeflected) $1 S$ atom. As field ionization is a tunneling process, its rate is highly sensitive to details of the antihydrogen wave function. Munger et al. estimate that the annihilation pattern produced by a sample of $1000 \bar{H}$ atoms should determine the mean radius of the $n=2 \bar{H}$ wave function to $\sim 0.3 \%$.

The E862 Lamb shift and fine-structure measurements follow in some respects an experiment described by Parkhomchuck (1988), on a beam of $65-\mathrm{MeV}$ hydrogen atoms. These were recombined from comoving electron and proton beams (as described later in this section) and were excited to $2 S$ states by passing them through a $0.1-\mu$ Formvar foil. The $2 S$ beam first moved through a $30-\mathrm{cm}$-long electrical condenser field of a few hundred $\mathrm{V} / \mathrm{cm}$. This induced a Stark perturbation so that the beam emerging from the condenser was in the superposed state $a|2 S\rangle+b|2 P\rangle$, the relative phase of $a$ and $b$ being determined by the condenser field, the atoms' transit time, and of course the Lamb shift $\mathcal{L}$ $=E\left(2 S_{1 / 2}\right)-E\left(2 P_{1 / 2}\right)$. The $2 P$ fraction decayed to $1 S$ with a time constant of $1.6 \mathrm{~ns}$ in the subsequent fieldfree drift space of length $1.5 \mathrm{~m}$, leaving only the metastable $2 S$ state. Finally, the metastable $2 S$ state was deexcited by a strong electric field; the $2 S-1 S$ photon detection rate then exhibited the oscillatory dependence of $a$ on the condenser voltage shown in Fig. 19.

Both complicating and simplifying factors occur in the transition from $65-\mathrm{MeV}$ protons $(\beta=0.35, \gamma=1.07)$ to antiprotons at some $9 \mathrm{GeV}(\beta \sim 1$ and $\gamma \sim 9)$, the antiproton beam energy of E862. The complicating factor is that in order to reproduce the Parkhomchuck conditions, the condenser would have to be some $10 \mathrm{~m}$ long for the same electric field, and the drift space several times the mean $2 P$ decay length of $\gamma \times c \times \tau_{2 P-1 S}$ $\sim 450.2 \mathrm{~cm}$. This is clearly impractical with the unfocused $\bar{H}$ beam.

Instead, the proposed measurement takes advantage of the close relationship between field ionization, as described above, and the Stark effect. The strong $(\sim 2$ $\left.\times 10^{9} \mathrm{~V} / \mathrm{cm}\right)$ motional electric field produced by the 0.7 $\mathrm{T}$ magnetic field following the excitation foil splits the $n=2$ manifold into a Stark triplet. Under these conditions (Mandelkern et al., 1997; Blanford et al., 1998b) the field-ionization rate depends strongly on the Stark

\footnotetext{
${ }^{20} 1 \mathrm{~S}$ atoms will not be ionized at all, while those with $n>2$ will ionize instantly.
}

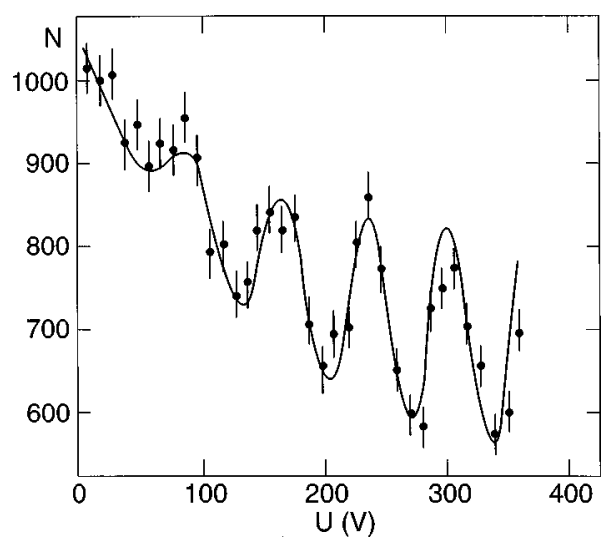

FIG. 19. Lamb-shift experimental results (see text) with 65MeV hydrogen beam, after Parkhomchuck (1988). The rate of detection of Lyman- $\alpha$ photons is displayed vs the voltage on the condenser (see text).

eigenstate, and the triplet can be labeled $\left|\Psi_{L}\right\rangle,\left|\Psi_{M}\right\rangle$ and $\left|\Psi_{S}\right\rangle$ in terms of the long, medium, and short fieldionization lifetimes of its members. These lifetimes (in the ratio 25:5:1) are such that any neutral atoms that emerge from the 30-cm-long 0.7-T magnetic field are in an almost pure $\left|\Psi_{L}\right\rangle$ state. In terms of component substates $|n, l, j\rangle$ this can be written

$$
\left|\Psi_{L}\right\rangle=\sqrt{\frac{1}{2}}|2,0,1 / 2\rangle+\sqrt{\frac{1}{6}}|2,1,1 / 2\rangle-\sqrt{\frac{1}{3}}|2,1,3 / 2\rangle .
$$

As antihydrogen atoms in the three substates have slightly different masses, the three waves will propagate with slightly different frequencies. During the atoms' flight through a field-free space, the components will therefore acquire relative phase changes, regenerating $\left|\Psi_{M}\right\rangle$ and $\left|\Psi_{S}\right\rangle$ states from the initial $\left|\Psi_{L}\right\rangle$ state as with similar regeneration effects in the $K^{0}-\bar{K}^{0}$ system. The regenerated amplitudes can be measured from the fieldionization pattern in a second "analyzing" magnet, approximately one $2 P$ decay length downstream.

In the neutral-kaon system, the regenerated amplitude can be used to determine the relative mass difference $\left(7 \times 10^{-15}\right)$ between $K_{L}$ and $K_{S}$. In the antihydrogen case (Fig. 20), the two regenerated amplitudes determine both the relative difference due to the Lamb shift $\left(4.7 \times 10^{-15}\right)$ and that due to the P-state fine structure $\left(\sim 7 \times 10^{-10}\right)$. With a drift space of one $2 P$ decay length, Mandelkern et al. then expect to reach a precision of $5 \%$ on the Lamb shift and $\sim 1 \%$ on the fine structure with about $10^{4}$ recombined atoms. This corresponds to little more than a few seconds of data acquisition under the 2000-H-atoms s ${ }^{-1}$ conditions of Parkhomchuck's hydrogen experiment, for which the Lamb shift precision (1\%) was not commensurately better. This apparently paradoxical situation is explained by the simplifying factor that the annihilation of the $\bar{p}$ (resulting from field ionization of the $\bar{H}$ atoms) can be detected with $100 \%$ efficiency, while the detection efficiency of VUV photons cited in Parkhomchuck (1988) is only $\sim 2 \times 10^{-3}$. 


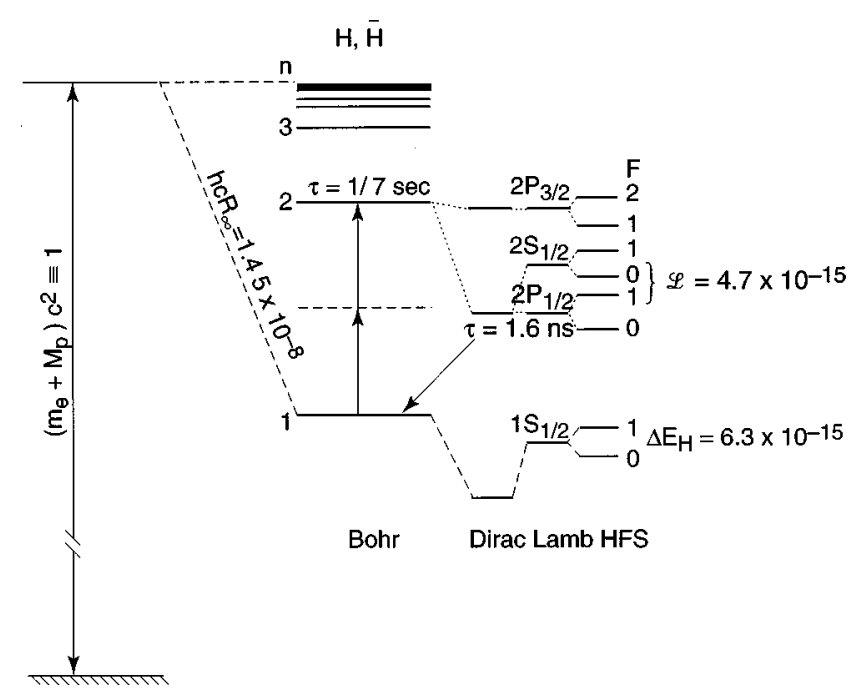

FIG. 20. Energy-level structure of the hydrogen and antihydrogen atoms. The ground-state binding energy $h c R_{\infty}, \mathrm{Lamb}$ shift $\mathcal{L}$, and ground-state hyperfine splitting $\Delta E_{H}$ are all normalized to the mass of the atom.

The interest of producing antihydrogen atoms at high energy is in the avoidance of the many technical problems (discussed in Secs. III and IV) associated with deceleration to energies $\ll h c R_{\infty}=13.6 \mathrm{eV}$, at which high recombination-reaction rates can be obtained. As it is the relative energy which determines the cross section, an alternative is to merge comoving, equal-velocity positron and antiproton beams in the same storage ring. This was recognized in the 1980s as a feasible, if difficult, goal for LEAR (Neumann et al., 1983, 1985; Wolf et al., 1986; Poth et al., 1988) and was indeed the production method used for ordinary hydrogen in the Parkhomchuck experiment described above. According to Bethe and Salpeter (1977), radiative recombination from the continuum,

$$
\bar{p}+e^{+} \rightarrow \bar{H}+h \nu
$$

and its $C P T$ conjugate (producing hydrogen) have cross section

$$
\sigma_{n}^{\text {spon }}=1.96 \pi^{2} \alpha^{3} a_{B}{ }^{2} \frac{1}{n} \frac{1}{1+n^{2} T^{*} / I_{0}} \frac{I_{0}}{T^{*}} .
$$

For final-state quantum number $n=1$ and center-ofmass energy $T^{*} \ll I_{0}=13.6 \mathrm{eV}, \quad \sigma_{1}^{\text {spon }}=2.1 \times 10^{-22}$ $\mathrm{cm}^{2} \times\left(13.6 \mathrm{eV} / \mathrm{T}^{*}\right)$. For higher $n$ the factor $n^{3}$ dominates for all but the lowest center-of-mass energies, but the cross section is still many orders of magnitude greater than that of Eq. (26). As we have seen, 2000 hydrogen atoms were synthesized per second by Parkhomchuck et al. with $40 \mu \mathrm{A}$ of $65-\mathrm{MeV}$ protons and an equalvelocity electron current of $0.3 \mathrm{~A}$. Moreover, rate increases of more than an order of magnitude were observed by Schramm et al. (1991) and Yousif et al. (1991) using laser stimulation of reaction [Eq. (29)], as suggested by Neumann et al. (1983, 1985), Wolf et al. (1986), and Poth et al. (1988). These methods must also be applicable to antihydrogen, and Meshkov (1997a, 1997b) and Meshkov and Skrinsky (1995) estimate an antihydrogen recombination rate of $10^{4} \mathrm{~s}^{-1}$ for $10^{11}$ stored $50-\mathrm{MeV}$ antiprotons and $10^{9}$ stored $27.2-\mathrm{keV}$ positrons.

Neumann $(1987,1988)$ discusses the possibilities for spectroscopic studies of antihydrogen atoms produced in the form of such swiftly moving beams. Even with laser stimulation, these beams will contain few atoms, and the time during which they can be kept under observation can clearly be no longer than their transit time through a reasonably sized experimental apparatus. ${ }^{21}$ As low-rate transitions (which via the uncertainty principle are just the ones that can be the most precisely determined) need long observation times, comparisons of hot hydrogen and antihydrogen atoms must always be of low precision, whether carried out by quantum interference or laser-spectroscopic means. The \%-scale precisions of the E862 quantum interference experiments and of the more recently proposed spectroscopic studies (Blanford et al., 1998c) of in-flight atoms, both to be tested with hydrogen atoms recombined from an $8-\mathrm{GeV}$ proton beam, reflect this overall limitation. It is for this reason that in the next section we return to a discussion of the physics of antihydrogen in the cold state.

\section{PHYSICS WITH COLD ANTIHYDROGEN}

The conclusion of Sec. VI must be that if the reason we measure the properties of the antiproton is to furnish a high degree of assurance on the $C P T$ symmetry principle that supposedly lies behind its very existence, the LEAR era was only a beginning in this work. A large part of the raison d'être for the AD machine is to furnish additional degrees of assurance via studies of the antihydrogen atom.

It is fortunate that the engine for antihydrogen research is of similar design to the one that has for some years driven studies of ordinary hydrogen and other atoms. Thus antihydrogen atoms held in a container made of ordinary matter will annihilate on collision with its walls; if they are to avoid this fate they must be levitated in neutral-atom traps. Fortuitously for antihydrogen physics, the same problem has had to be solved in BoseEinstein condensation (BEC) experiments in order to eliminate three-body recombination of spin-down monoatomic hydrogen atoms with helium-coated container walls (Setija et al., 1993). BEC and other studies have also seen the development of the Lyman- $\alpha$ cooling techniques that will be needed for antihydrogen studies, while the rapid increase in the precision of $1 S-2 S$ laser spectroscopy in hydrogen has come about partly because these atoms can be confined at very low temperatures in neutral traps or cold atomic beams (Hänsch and Zimmermann, 1993; Weitz et al., 1994; Cesar et al., 1996). Even the enormous difference in availability of antihydrogen and hydrogen atoms is largely irrelevant in certain shelving experiments, where a few atoms, continu-

\footnotetext{
${ }^{21}$ In the unstimulated CERN experiment the antiatoms traveled only $15 \mathrm{~m}$ and survived for only $\sim 40 \mathrm{~ns}$ before disassociation.
} 
ously driven around a closed cycle of atomic states, make the same sequence of transitions on each circuit; Hansch and Zimmermann (1993) have suggested an experimental comparison of the $H-\bar{H} 1 S-2 S$ frequencies that would require only $\sim 1000$ trapped atoms of each kind at a temperature of $0.2 \mathrm{~K}$, cycled 60 times.

The trend of these techniques is of course towards ever higher precision via the reduction of such systematic limitations as transit-time broadening and Doppler and Zeeman effects. It is safe to assume that while the specific problems of synthesizing antihydrogen atoms are being solved, progress will continue on the hydrogen atom alone toward quantum-limited precision in the $1 S-2 S$ transition. We therefore consider below only those problems specific to antihydrogen.

\section{A. Positron sources}

For antihydrogen production we will clearly need a supply of positrons. These are readily produced from radioactive sources such as ${ }^{22} \mathrm{Na}$,

$$
{ }^{22} \mathrm{Na} \rightarrow{ }^{22} \mathrm{Ne}^{*}+e^{+}+\nu+0.545 \mathrm{MeV},
$$

which has a half-life of $2.6 \mathrm{y}$, or from electron accelerators via electromagnetic showers:

$$
\begin{aligned}
& e^{-}+(Z, N) \rightarrow e^{-}+\gamma \\
& \gamma \rightarrow e^{+}+e^{-} .
\end{aligned}
$$

Both produce positrons over a broad range of energies that extends up to $\mathrm{MeV}$ values. As with antiproton production, this is in sharp conflict with any scheme for confining the particles in traps. The minute fraction of positrons at the extreme low-energy end of the spectrum can, however, be considerably increased by thermalizing the positrons in a solid moderator placed near, or sometimes in contact with, the source; those which reach thermal energies in a thin layer near the moderator surface can then be reemitted at $\mathrm{eV}$ energies with an efficiency $\epsilon \equiv I_{\mathrm{eV}} / I_{\text {source }}$, depending on the moderator substance and the work function of its surface (Charlton and Laricchia, 1993). This is possible because the typical time for annihilation is of the order of $100 \mathrm{ps}$, while thermalization takes place in a few ps. Single-crystal metallic moderators have been used either in a reflection geometry (i.e., with the $\mathrm{eV}$ positrons leaving the surface through which they entered) or in transmission geometry, in which case they enter one side of a thin foil and leave it through the other. Typical $\epsilon$-values for metal moderators are a few $\times 10^{-4}$. Recently the technique of freezing solid noble-gas moderators directly onto the surface of positron-emitting sources (Mills and Gullikson, 1986) has resulted in an order-of-magnitude improvement in $\epsilon$.

Normally the eV positron flux from a moderately strong (tens of $\mathrm{mCi}$ ) source is so far in excess of typical antiproton fluxes that these low overall efficiencies do not matter. It is thus generally technologically easier, as well as cheaper, to introduce $\mathrm{eV}$ positrons into a trap from a nearby source than to reproduce at electron ac-

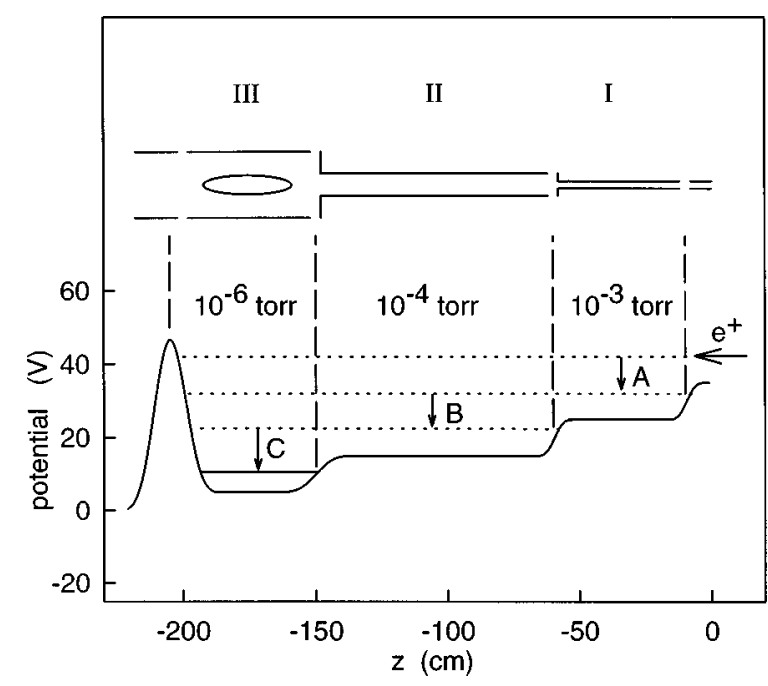

FIG. 21. Trap (see text) for positrons arriving from a continuous source, developed by Greaves and Surko (1997). The cylindrical Penning-Malmberg electrode geometry (above) produces the potential steps of regions A, B, C (below). These are maintained at successively lower buffer gas pressures, and a magnetic field of $1 \mathrm{kG}$ is aligned on the horizontal axis. Diagram kindly supplied by C. M. Surko.

celerators the more efficient but also more complex antiproton deceleration schemes. The simple expedient of loading the trap by switching the electrode potentials (as is done with bunched antiprotons, discussed in Sec. IV.A.1) will not, however, work with randomly emitted source positrons. These, having found their way into the trap, cannot remain there for long unless a dissipative force can be introduced before they find their way out again. Greaves and Surko (1997) introduce dissipation via collisions of the positrons with a differentially pumped nitrogen buffer gas in the three sections of the trap shown in Fig. 21. This procedure results in the accumulation of positrons into the deepest section of the trap. Naturally, the buffer gas introduces annihilation processes as well as dissipation even in this section. Figure 22 (top) shows that an equilibrium is eventually established between annihilation losses and loading rates. The former can be seen as a 60-s decay curve of the stored positrons when the source is removed (Fig. 22, bottom). If the buffer gas feed is also turned off, the pressure drops by some three orders of magnitude in $\sim 30 \mathrm{~s}$ and the annihilation loss time constant increases to 30 minutes. The trap contents can then be loaded into a valved-off ultralow-pressure recombination vessel by opening the valve and removing the potential barrier at the left of Fig. 21. These figures demonstrate that a loading rate of some $3 \times 10^{7} e^{+} \mathrm{s}^{-1}$ can be obtained with a relatively modest $65 \mathrm{mCi}$ source.

In a second method developed by Schwinberg et al. (1981), positrons were introduced without moderation from a source into a trap that was carefully biased to ensure that a few of them followed trajectories that would carry them out again only after a complete magnetron period $(\sim 100 \mu \mathrm{s})$. During this time (van Dyck, Schwinberg and Dehmelt, 1987) sufficient energy could be coupled out to a resonant tuned circuit (as described 

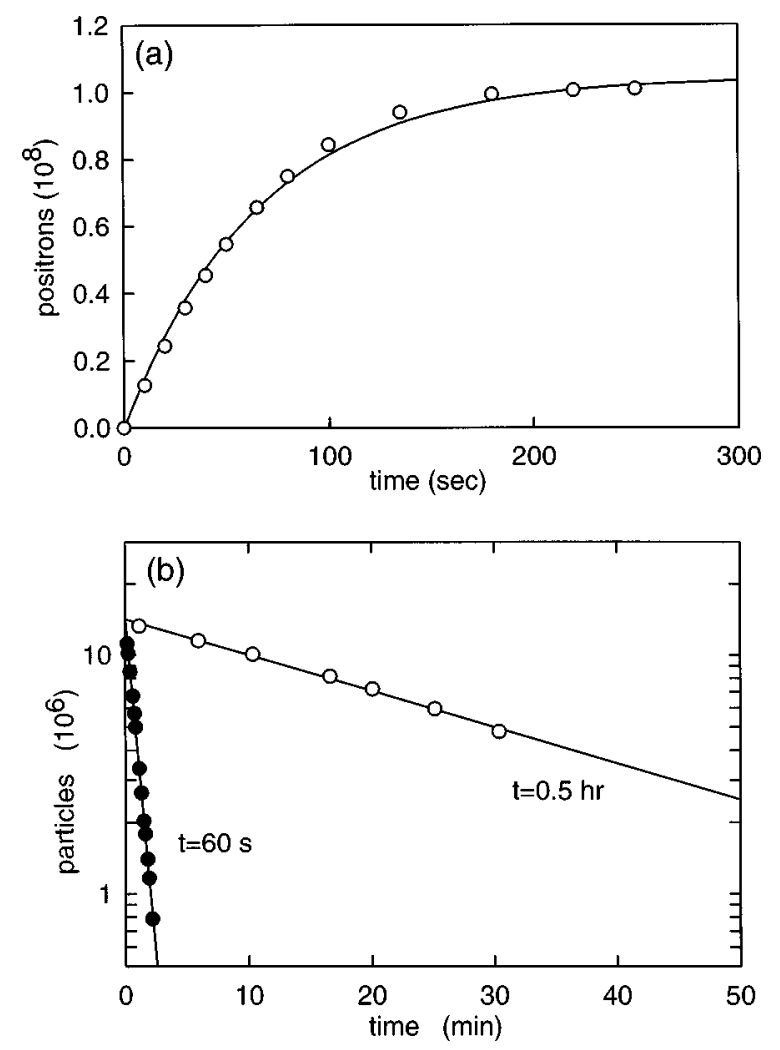

FIG. 22. Loading (top) and storage (bottom) of the positron trap of Fig. 21. The short and long decay constants are obtained with and without buffer gas feeding, and after the positron source is removed. Diagram kindly supplied by C. M. Surko.

in Sec. IV.A.3 for antiprotons) to keep these few positrons inside the trap permanently. By combining this technique with tungsten crystal moderation, Haarsma et al. (1995) obtained a loading rate from a $5 \mathrm{mCi}$ source of $3 \times 10^{4}$ per day. A recent development is the capture of positrons from the ionization of $P s$ atoms, emitted from the tungsten crystal (as are the positrons) by their positive work function; this has increased the accumulation rate by a further factor of 50 to some $10^{6}$ per day (ATRAP, 1997). Further increases in accumulation rate to $10^{6}$ per hour may be obtained simply by installing a larger $(150 \mathrm{mCi})$ source. There is also the possibility (Wineland et al., 1993) of introducing dissipative collisions with a ${ }^{9} \mathrm{Be}^{+}$ion plasma at a density of $\mathcal{O}\left(10^{9}\right)$ ions per $\mathrm{cm}^{3}$ (ATRAP, 1997). This plasma would be loaded in advance of the positrons and cooled by laser techniques to $10 \mathrm{mK}$. Coulomb repulsion between the like-charge species largely eliminates positron annihilation in this case, so that no pumping of the plasma is necessary.

\section{B. Recombination reactions}

We now have to consider how to produce antihydrogen atoms given that a supply of antiprotons and positrons has been made available in thermal equilibrium at $4.2 \mathrm{~K}$ in Penning or Penning-Malmberg traps by the

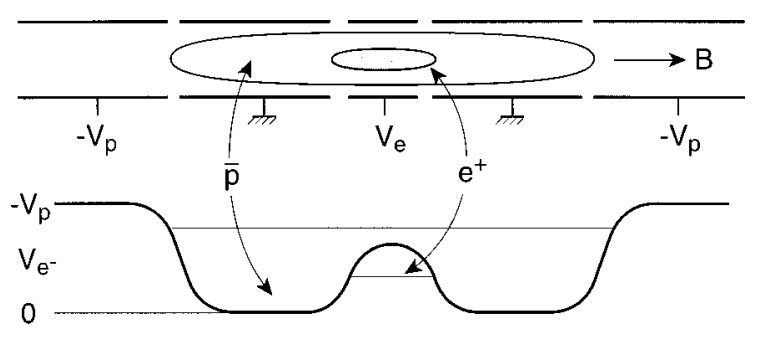

FIG. 23. Nested trap for joint confinement of positively and negatively charged particles, after Gabrielse. As with the positron trap of Fig. 21, the electrodes (above) have the cylindrical Penning-Malmberg geometry, and produce potential wells (below) of both signs.

methods described above. As traps for positive particles reject negative ones, it will be necessary to enclose both of these samples in some variant of the normal trap that can confine particles of both signs in a small volume. Two solutions have been suggested for this, the nested Penning-Malmberg trap (Brown et al., 1997) and the combined Paul and Penning trap ( $\mathrm{Li}$ and Werth, 1992; Walz et al., 1995).

A nested Penning-Malmberg trap is a positron Penning-Malmberg trap at the center of one containing antiprotons, as schematically shown in Fig. 23. This is possible because potential wells for oppositely charged species are of opposite sign. The principle has now been demonstrated for positrons and antiprotons (ATRAP, 1997) as well as for electrons and protons (Hall and Gabrielse, 1996). In the latter case, protons passing through the electron cloud during their excursions through the inner electron potential well lose energy by collisions until they settle into their own outer wells (the electrons of course radiate this energy efficiently, as we have discussed in Sec. IV.A.3). Gabrielse et al., (1989) have already demonstrated the cooling effect of collisions between antiprotons and electrons in singlespecies Penning-Malmberg traps. To ensure continued cooling in the double-species trap, the positron well depth would be adiabatically reduced until both species reached thermal equilibrium with their surroundings.

In the combined Paul-Penning trap, antiprotons are confined by the usual Penning-trap fields of Fig. 9. This configuration will plainly eject the oppositely charged positrons. Microwave radiation is therefore supplied to the trap volume, to add an oscillating electric quadrupole field that is strong enough to overcome this tendency for the positrons, but is too weak to affect the more slowly moving antiprotons. The principle has been demonstrated by Walz et al. (1995) with electrons and $\mathrm{H}_{2}{ }^{+}$ions (together with some protons).

Once enclosed in the same volume, the antiprotons and positrons can recombine via several chemical reactions:

$$
\begin{aligned}
& \bar{p}+e^{+}+e^{+} \rightarrow \bar{H}(n, l)+e^{+}, \\
& \bar{p}+e^{+} \rightarrow \bar{H}(n, l)+h \nu, \\
& N h \nu+\bar{p}+e^{+} \rightarrow \bar{H}(n, l)+(N+1) h \nu,
\end{aligned}
$$




$$
\bar{p}+\operatorname{Ps}\left(n_{1}, l_{1}\right) \rightarrow \bar{H}(n, l)+e^{-} .
$$

All of these have cross sections that increase rapidly as the relative velocity of the constituent particles falls, making it necessary to cool these to thermal or subthermal temperatures and plasmalike densities. In the case of the nested trap, further reduction of the positron well depth at equilibrium should then trigger the recombination by bringing the oppositely charged species into contact once more. The combined trap of course maintains them in constant contact during and after cooling to equilibrium, but in neither case have tests yet been done that show clear evidence for recombination. Furthermore, the necessary stability of protons passing through the electron cloud in the nested trap has not yet been shown, and the possible reduction of the reaction rate in the combined trap resulting from rf heating of the positrons is not well understood.

We have already encountered Eq. (34) in Section VI.D, when considering recombination in almost-equalvelocity comoving beams, and noted that its cross section for recombination to the $n=1$ state $\left[\sigma_{1}{ }^{\text {spon }}=2.1\right.$ $\times 10^{-22} \times\left(13.6 / T^{*}\right) \mathrm{cm}^{2}$ at relative energy $\left.T^{*}\right]$ can be increased by laser stimulation [Eq. (35)] by a factor of 10-100. The small scale of $\sigma_{1}{ }^{\text {spon }}$ relative to $\sigma^{\text {geom }} \sim 10^{-16} \mathrm{~cm}^{2}$ is just a reflection of the well-known fact that the time required for a $13.6-\mathrm{eV}$ electron to radiate a photon is about $1 \mathrm{~ns}$, while the time it spends within a few Bohr radii of the proton is only $\sim 10^{-15} \mathrm{~s}$. For $N_{\bar{p}}$ antiprotons and $N_{e^{+}}$positrons, both trapped in a common volume $V$ and with relative velocity $v^{*}$, the reaction rate $\Gamma_{\text {spon }}$ is

$$
\Gamma_{\text {spon }}=N_{e^{+}} N_{\bar{p}} \sigma_{1}^{\text {spon }^{*}} v^{* / V} .
$$

In a practical trap environment, actual rates depend on the degree of overlap of the plasmas, their fractional time of contact and their internal velocity distributions, as well as on disassociation rates via collisions or ionization by the trap fields. With $10^{7}$ antiprotons, $10^{8}$ positrons, and perfect overlap in space and time, an upper limit of $\Gamma_{\text {spon }}=7 \times 10^{3} \mathrm{~s}^{-1}$ at positron energy $10 \mathrm{meV}$ is estimated by the ATHENA group (ATHENA, 1997). This could be expected to increase by a factor of 100 if recombination were stimulated to the $n=2$ state with a $20-\mathrm{MW} / \mathrm{cm}^{2}$ pulsed $\mathrm{CO}_{2}$ laser, as discussed in Sec. VII.D (Schramm et al., 1991). Ever-higher laser powers do not produce ever-higher enhancement factors as they also increase the photo-reionization rate.

The three-body reaction [Eq. (33)] is interesting because estimated rates become extremely large at low energies. On the other hand its cross section $\sigma_{2 e^{+}}$is proportional to $n^{6} .^{22}$ It therefore populates fragile Rydberg states most abundantly, and many of these may not survive a long process of deexcitation to the more interesting low- $n$ states. Again because two positrons are in-

\footnotetext{
${ }^{22}$ Semiclassically, two positrons must collide simultaneously with the antiproton within an annulus $d r$; the probability for each of these collisions is $d \sigma / d n=2 \pi r_{n} d r$, with $r_{n} \propto n^{2}$.
}

volved, its rate dependence (Gabrielse, Rolston, and Haarsma, 1988) on $N_{e^{+}} / V$ is quadratic,

$$
\Gamma_{2 e^{+}} \sim\left(N_{e^{+}} / V\right)^{2}\left(1 / T^{*}\right)^{4.5}
$$

making high positron densities mandatory. These drawbacks are compensated for by the rapid inverse dependence on the relative energy; thus the ATHENA Collaboration (1997) gives $\Gamma_{2 e^{+}} \sim \Gamma_{\text {spon }}$ at the $10-\mathrm{mV}$ positron energy mentioned above and $\Gamma_{2 e^{+}} \gg \Gamma_{\text {spon }}$ at still lower energies.

The remaining possibility [Eq. (36)] entails somewhat easier trapping and cooling problems as (a) the $P s$ atoms need only be emitted into a single-species trap containing antiprotons, and (b) the ground-state cross section remains $\mathcal{O}\left(10^{-15}\right) \mathrm{cm}^{2}$ up to $\mathrm{eV}$ energies. In addition, and in contrast to the case of reaction [Eq. (33)], the $C P T$-conjugate reaction has now been experimentally demonstrated (Merrison et al., 1997). Its principal problem is an extremely low recombination rate $\left(\sim 10 \mathrm{~h}^{-1}\right.$ per $10^{10}$ low-energy positrons delivered from a separate accumulator to a $P s$-forming solid surface). It may, however, be possible to increase this figure by many orders of magnitude, if the $P s$ atoms are first laser-excited to $n_{1} \sim 10$.

\section{Spectroscopy}

Partly because of the immense power of modern laser and maser techniques and partly because of the development of neutral-atom traps and cold atomic beams, it has become almost commonplace to see phenomenally accurate measurements on the hydrogen atom reported in the literature. On the other hand, antihydrogen spectroscopy is (at the moment) like exobiology-a discipline without any subject matter. We cover briefly below what are likely to be the main points of interest when this situation changes. Somewhat more detail can be found in the review by Charlton et al. (1994), while modern hydrogen spectroscopy is treated in depth by $\mathrm{Pa}-$ chucki et al. (1996).

The main features of the hydrogen and antihydrogen energy-level structure are shown in Fig. 20, where all energy differences are expressed dimensionlessly as a fraction of the mass of the atom. The common scaling factor for all frequencies is the Rydberg constant $R_{\infty}$. This is the most important property determined by the larger energy intervals such as those from $1 S$ to $2 S$ and $1 S$ to $2 P$, although the reduced mass of the positron or electron enters at the $0.5 \%$ level. We have noted that a high precision null comparison of the $1 S-2 S$ frequencies in the $C P T$-conjugate atoms would be particularly fruitful. The fastest way the $2 S$ atom can deexcite to the ground state is via the emission of two photons, as angular momentum conservation forbids single $E 1$ dipole transitions (magnetic dipole single-photon emission has a decay constant of two days). The deexcitation is inherently very slow: its $\sim 1 / 7 \mathrm{~s}$ lifetime corresponds by the uncertainty principle to a linewidth of $1 \mathrm{~Hz}$ in $10^{15}$. However, two-photon spectroscopy is a practical advantage rather than a hindrance, since if the atom absorbs 
two counter-propagating photons, the first-order Doppler effect is zero; already, in the hydrogen case, the two-photon technique has opened a path towards quantum-limited experiments. The $1 S-2 S$ line center in such an experiment might ultimately be found to one part in $10^{3}$ of its width, raising the prospect of a $C P T$ test with antihydrogen at the $10^{-18}$ precision. For hydrogen, the cold-beam result of Udem et al. (1997) referred to in Sec. IV.C.2 is

$$
\nu_{1 S-2 S}(H)=2466061413.18734(84) \mathrm{MHz} .
$$

For completeness, the two-photon spectroscopy results for $P s$ and $M u$ (Fee et al., 1993; Maas et al., 1994) are

$$
\nu_{1 S-2 S}(M u)=2455529002(80) \mathrm{MHz}
$$

and

$$
\nu_{1 S-2 S}(P s)=1233607 \text { 216.4(3.2) MHz. }
$$

These values are less precise than those in hydrogen and are likely to remain so for reasons given in Sec. V.A.

While the $1 S-2 S$ frequency may until now have received the most attention as a benchmark experiment for antihydrogen, it by no means exhausts the possibilities of this atom as a $C P T$ laboratory; carefully designed studies of other spectroscopic features would permit its properties to be determined with high precision. For hydrogen the ground-state hyperfine structure $\Delta E_{H}$ is now known to about one part in $10^{12}$ experimentally; its quantum width is so small that it will probably never be reached experimentally. Moreover, the hyperfinestructure frequency directly measures the product of $\bar{p}$ and $p$ magnetic moments. Colladay and Kostelecký (1997) present a specific CPT- and Lorenz-invarianceviolating extension of the standard model that exhibits $H / \bar{H}$ differences in both $1 S-2 S$ and hyperfine Zeeman transitions. Hyperfine-structure comparisons of $H$ and $\bar{H}$ will thus be informative indicators of $C P T$ violation even when done with relatively low-precision "classical" atomic-beam techniques; done by standard microwave resonance techniques, they should provide extremely-high-precision comparisons (Nafe and Nelson, 1947). $1 S-2 S$ frequency comparisons, on the other hand, test the theorem via the Rydberg constant, i.e., in the relatively well-verified domain of $\bar{p}$ and $e^{+}$masses and charges, implying that the highest-precision comparisons, although undoubtedly technically possible, will also be mandatory if one is to learn anything new.

Other possibilities are the $2 S$ and $1 S$ Lamb shifts $\mathcal{L}$, which include radiative and vacuum polarization terms, the $1 S-2 P$ (1.6-ns-lifetime) Lyman- $\alpha$ transition, and transitions between Rydberg states. The proviso just made concerning the $1 S-2 S$ transition will of course apply equally to all these.

Spectroscopic experiments with antihydrogen differ from those made with hydrogen in two important respects. The first is the ease with which the recombination reaction itself produces excited atoms: Rydberg states will be populated preferentially in reaction (33) (although they may soon be lost again), $\mathrm{CO}_{2}$ laser- stimulated recombination [Eq. (35)] will initially produce samples of $n=12$ or $n=11$ states, and recombination via $P s$ [Eq. (36)] populates low-lying $n=2$ and $n$ $=3$ states as efficiently as it does the ground state. It is not necessary, as is the case with hydrogen, to start with the ground state; this is clearly an advantage when few antiatoms are available.

The second difference is that antiparticle and antiatom annihilations, being cataclysmic events on the atomic scale, are easy to see. In Sec. IV.B.5 we noted how certain transitions in antiprotonic helium could be detected with $\sim 100 \%$ efficiency via synchronous annihilation of the antiproton; it would hardly have been possible to detect photons emerging into a $4 \pi$ solid angle. It is possible to think of a number of ways to signal resonant transitions in trapped antihydrogen atoms by the appearance of annihilation. One example is to quench the resonant $2 S$ state to $2 P$ on the $1 S-2 S$ resonance; spontaneous deexcitation to the high-field-seeking $1 S$ hyperfine ground state would then release the atom from the trap and allow it to annihilate on the walls. The ground-state hyperfine structure itself could also be measured by detecting the transition from a high-fieldseeking (trapped) state to the low-field one. Photoionization to the continuum is of course possible from any state with $n \geqslant 2$.

However, as far as comparative hydrogenantihydrogen measurements at the highest precision are concerned these advantages are a double-edged sword, in that they introduce procedural differences between the $C P T$-conjugate atoms under study. In particular, detection by annihilation is not available at all for hydrogen. Extremely precise null comparisons relying on either of these points will therefore become impossible just when the precision reaches the level at which the null method is most needed.

\section{Evaluation of antihydrogen results in terms of $C P T$}

The claim that hydrogen-antihydrogen comparisons can furnish a meaningful degree of assurance that nature exhibits $C P T$ symmetry must clearly be justified against similar claims made on behalf of rival particleantiparticle systems.

So far the only test at the $10^{-18}$ level (suggested above as the goal of $1 S-2 S$ comparisons) is the one deduced from measurements of $C P$-violation parameters in the neutral-kaon system. Any $C P T$ violation manifesting itself as a $K^{0}-\bar{K}^{0}$ mass difference must also contribute an additional complex amplitude $\delta$ to the small $C P$-violating admixture $\epsilon$ in $K_{L}$ and $K_{S}$. The component of $\delta$ perpendicular (in the complex plane) to $\epsilon$ and the phase $\phi_{\epsilon}$ of $\epsilon$, are related to the mass difference by

$$
\frac{\left[m\left(K^{0}\right)-m\left(\bar{K}^{0}\right)\right]}{\left[m\left(K_{L}^{0}\right)-m\left(K_{S}^{0}\right)\right]} \sim\left(2 \delta_{\perp} / \sin \phi_{\epsilon}\right) .
$$

Carosi et al. (1990) deduced $\delta_{\perp}$ from measurements of $C P$-violating amplitudes in $K^{0} \rightarrow \pi^{+} \pi^{-}$and $K^{0} \rightarrow 2 \pi^{0}$ decays to obtain an upper limit of

$$
\left[m\left(K^{0}\right)-m\left(\bar{K}^{0}\right)\right] / m\left(K^{0}\right) \leqslant 5.0 \times 10^{-19}
$$


on hypothetical $C P T$ violation in the $K^{0}-\bar{K}^{0}$ system. Assuming no $C P T$ violation elsewhere than in $K^{0}-\bar{K}^{0}$ mixing, the CPLEAR Collaboration has derived a value of $1.06 \times 10^{-18}$ (Adler et al., 1997) for this limit from their own measurements of $C P$-violation parameters. Yet how meaningful are these values in the light of the discussions of Sec. V.A.? One might just as well use the known $K_{L}-K_{S}$ mass difference,

$$
\left[m\left(K_{L}\right)-m\left(K_{S}\right)\right] / m\left(K^{0}\right)=7 \times 10^{-15},
$$

as a reference value and obtain instead of Eq. (43) the limit

$$
\left[m\left(K^{0}\right)-m\left(\bar{K}^{0}\right)\right] /\left[m\left(K_{L}\right)-m\left(K_{S}\right)\right] \leqslant 2.6 \times 10^{-2} .
$$

It is usual to quote the impressive limit (43) to indicate the present status of the $C P T$ theorem. However, as noted at the head of Sec. V.A, Kobayashi and Sanda (1992) point out that as $m_{\bar{K}^{0}} \neq m_{K^{0}}$ requires both $C P T$ and $C P$ violation, Eq. (42) is the more realistic measure of $C P T$ invariance, and that if the theoretical error on $\phi_{\epsilon}$ is taken into account, the limit (45) ought to be further relaxed to about $10 \%$.

The next best $C P T$ tests from the static properties of antiparticles are the $e^{+} e^{-} g$-factor comparison $\left[g\left(e^{+}\right)\right.$ $\left.-g\left(e^{-}\right)\right] / g\left(e^{+}\right) \leqslant(0.5 \pm 2.1) \times 10^{-12}$ (van Dyck, Schwinberg, and Dehmelt, 1987) and the $p-\bar{p}$ cyclotron frequency comparison $\left[e_{\bar{p}} / m_{\bar{p}}-e_{p} / m_{p}\right] /\left(e_{p} / m_{p}\right) \leqslant 1.5$ $\times 10^{-9}$ (Gabrielse et al., 1995). Seen from the standpoint of Eq. (43), these values seem quite tame, all the more so when it is considered that the $(g-2)$-values should really be compared instead of the $g$ values themselves. Finally, as Hughes and Deutch showed, the comparison of proton and antiproton cyclotron frequencies $\omega_{c}=e$ $\times B / m_{\bar{p}}$ to 1.5 parts in $10^{9}$ is not strictly a mass or charge test of $C P T$ invariance. By combining it with the rather poorly known antiproton Rydberg constant, it can be interpreted as a test of either quantity, but at the much lower precision of one part in $10^{5}$. Even if the $\mathrm{e} / \mathrm{m}$ ratio comparison improves to one part in $10^{11}$, as may happen in the $\mathrm{AD}$ era, there will be no spectacular increase in precision on $q_{\bar{p}}$ or $m_{\bar{p}}$ until the antiproton Rydberg is also measured to one part in $10^{11}$.

Turning now to the $H-\bar{H}$ system, we first recall that any lifting of degeneracy reveals an underlying lack of symmetry. Thus the $K_{L}$ and $K_{S}$ mass difference comes about because of degeneracy lifting by the weak interactions; its small scale just means that the weak-interaction symmetry violation is a small dynamical detail relative to the $q \bar{q}$ QCD force (Barut, 1987).

This suggests that we look for a dynamical detail in the lepton-hadron QED force of about the same magnitude to scale possible $C P T$-violation effects in the $H$ $-\bar{H}$ system. The ground-state splitting of hydrogen discussed in the previous section is just such a detail. Expressed in dimensionless terms as in Eq. (43) its value is (Hellwig et al., 1970; Essen et al., 1971)

$$
\left[m\left(H_{F=1}\right)-m\left(H_{F=0}\right)\right] / m\left(H_{F=1}\right)=6.3 \times 10^{-15} .
$$

The frequency itself is known to better than one part in $10^{12}$, but the theoretical value is considerably less accurate. The degeneracy lifting of Eq. (46) of course comes from symmetry violation by the dipole-dipole force under independent rotations of these dipoles and can be used to scale hypothetical $C P T$ violation in the $H-\bar{H}$ system, as Eq. (44) does in the $K^{0}-\bar{K}^{0}$ system. The weakness of the hyperfine splitting does not of course come from weak interactions but from the combined weaknesses of (a) the relativistic effect we know as magnetism, (b) the proton's magnetic dipole (compared with that of the electron), and (c) the magnetic dipole-dipole force itself.

While the similarities in scale between the hyperfinestructure and $K_{L}-K_{S}$ splittings should not be taken too literally, they do suggest that the $H-\bar{H}$ lepton-hadron system and the $K^{0}-\bar{K}^{0}$ quark-antiquark system are at least on an equal footing as $C P T$ laboratories: details in the interaction of their components have in both cases already revealed extremely important symmetry violations. In assessing their relative merits, we could point out that as the neutral kaons are already $q \bar{q}$ systems, a source of asymmetry in matter-antimatter masses is $a$ priori perhaps more likely to be found in systems consisting wholly of quarks and/or leptons (Zichichi, 1996). A further point in antihydrogen's favor is that the $C P T$ test in the neutral-kaon system is not assumption-free, as it depends on a dynamical model linking the measured $C P$-violation parameters with the elements of the mass matrix.

Taking all these considerations together, the present $C P T$-violation limit in the $K^{0}-\bar{K}^{0}$ system is best seen as a yardstick for future $C P T$ tests, not as an argument against making them.

\section{E. Evaluation of antihydrogen results in terms of gravitation}

We take up again here the discussion of Sec. V.B, where we concluded that an empirical vacuum with respect to the gravitational properties of antiparticles needs to be filled. Ballistic experiments to this end (all so far unsuccessful) were described in Sec. VI.C, ${ }^{23}$ where we noted that comparisons of the ballistic properties of $e^{+}$and $e^{-}$and of $p$ and $\bar{p}$ must always be severely influenced by systematic errors arising from stray electric and magnetic fields. An alternative, nonballistic, approach concerns the behavior of antihydrogen clocks. Clocks, as Morrison reminds us (1958), are troublesome contrivances in gravitational fields. Each antihydrogen atom constitutes a microscopic clock made of antimatter, ticking with an entire sequence of eigenfrequencies

\footnotetext{
${ }^{23}$ An experiment has also been suggested in which a quantum interferometer would measure the gravitational acceleration of a horizontally propagating cold antihydrogen beam (Phillips, 1995, 1997), as has been done with neutrons and neutral atoms. Space considerations preclude a discussion of this interesting idea here.
} 
$\sim h c R / h^{2}$ when wound up by excitation from the ground state. The effect of a local gravitational field is to introduce a gravitational redshift in all these tick rates.

Hughes (1993a) and Hughes and Holzscheiter (1992) have used Thirring's approach to gravitation (Thirring, 1961, 1969) to exhibit the difference in the redshifts of hydrogen and antihydrogen clocks which would accompany an equivalence-principle violation by antimatter. ${ }^{24}$ In general relativity, the Newtonian gravitational potential $\phi=G Q_{E} / r$ at a distance $r$ from a point or spherical source of strength $Q_{E}$ is replaced by a symmetric $4 \times 4$ metric tensor $g_{\mu \nu}$. The relation between Newtonian and general relativity potentials is $\phi=c^{2} / 2\left(g_{00}-1\right)$, where $g_{00} \equiv 1+h_{00}$ and $h_{\mu \nu}$ is the gravitational field tensor. In the weak-field limit, which includes only terms up to $\mathcal{O}\left(v^{2} / c^{2}\right)$, off-diagonal elements can be ignored, although we still have to include non-Newtonian terms in $g_{11}, g_{22}$, and $g_{33}$. Hughes' approach further restricts attention to cases in which spin effects can be ignored, and in which $v / c \ll 1$ for the electron. All these assumptions are justified for experiments carried out on electric $n$-pole transitions of hydrogen and antihydrogen atoms at nonsingular points in the solar system.

As the field $h_{\mu \nu}$ couples to the energy-momentum tensor $T^{\mu \nu}$, the Lagrangian density is then just the contraction $h_{\mu \nu} T^{\mu \nu}$ and can be split into parts corresponding to the matter field of the electron $T_{e}^{\mu \nu}$ and the electromagnetic force field of the proton $T_{E M^{\mu \nu}}{ }^{25}$

Under the above assumptions the interaction density (Bell, 1987) splits into matter and charge parts, $L_{m}$ and $L_{q}$, such that

$$
\begin{aligned}
L_{m}= & \int_{\Omega} d t\left[-\left(1+\phi / c^{2}\right) m_{e} c^{2}\right. \\
& \left.+\left(1-3 \phi / c^{2}\right) \frac{1}{2}\left(v^{2} / c^{2}\right) m_{e} c^{2}\right]
\end{aligned}
$$

and

$$
L_{q}=\int_{\Omega} d^{4} x\left[\left(1-2 \phi / c^{2}\right) \mathbf{E}^{2}-\left(1+2 \phi / c^{2}\right) \mathbf{B}^{2}\right] .
$$

Equation (48) is analogous to the frequency change of an electrical resonant circuit or cavity due to the spacetime distortion introduced by a gravitational field. This can be thought of as a change of the dielectric constant of free space, its permittivity and permeability $\epsilon_{0}$ and $\mu_{0}$ being replaced by local values $\epsilon_{0}\left(1-2 \phi / c^{2}\right)$ and $\mu_{0} /(1$ $\left.-2 \phi / c^{2}\right)$, where $\phi$ is the local gravitational potential. ${ }^{26}$ Were Eq. (48) alone to apply, we would observe a gravitational blueshift in such frequencies. Equation (47) is a

\footnotetext{
${ }^{24}$ The authors caution that abandoning the equivalence principle inescapably leads to an absolute significance for $\phi$, so that observables like particle masses depend on where they are in the universe.

${ }^{25} \mathrm{~T}_{e}^{\mu \nu}=m_{e} c^{2} \gamma d x^{\mu} / d t \times d x^{\nu} / d t$; the $T_{E M}^{\mu \nu}$ part is defined in terms of the electromagnetic field strength tensor $F^{\alpha \beta}$. See Jackson (1975), p. 550.

${ }^{26}$ Constant over atomic distances.
}

larger inertial effect replacing the mass $m$ of a particle by $m\left(1-3 \phi / c^{2}\right)$ and changing the overall effect from a blueshift to a redshift.

Equations (47) and (48) differ from the zero-field values only via these additional factors; consequently the equation of motion resulting from the action principle is just the Schrödinger equation ${ }^{27}$ modified by the inclusion of similar factors. We can then directly replace $m$ and the fine-structure constant $\alpha=e^{2} / 4 \pi \epsilon_{0} \hbar c$ by the corresponding factors in the $1 S-2 S$ atomic transition frequency $\hbar \omega_{12}=1 / 2 m \alpha^{2} c^{2}\left(1 / 1^{2}-1 / 2^{2}\right)=3 / 8 m \alpha^{2} c^{2}$ to give

$$
\hbar \omega_{12}(\phi)=3 / 8 m\left(1-3 \phi / c^{2}\right) \alpha^{2}\left(1+4 \phi / c^{2}\right) c^{2} .
$$

We then get the result

$$
\omega_{12}(\phi) \approx\left[1+\phi / c^{2}\right] \omega_{12}(0),
$$

showing that the atom behaves like an elementary clock with redshift $\Delta \omega / \omega=\phi / c^{2}$, or equivalently that the weight of the $1 S-2 S$ energy difference is in proportion to its mass. These are the not unsurprising consequences of having implicitly assumed the validity of the weakequivalence principle.

Now if the principle is not valid for antimatter, gravitational fields will affect the time-keeping properties of elementary antihydrogen clocks differently from the way it affects those of elementary hydrogen clocks. ${ }^{28}$ Hughes introduces this possibility by allowing antihydrogen to experience the usual tensor coupling but with an anomalous strength so that its acceleration becomes $f g,(f \sim 1)$. In this case $\phi=\int_{\infty}^{r} g d r$ becomes $f \phi$ in $L_{m}$ resulting in a new transition frequency $\bar{\omega}_{12}$ for $\bar{H}$ :

$$
\omega_{12}(\phi)=\left[1+(4-3 f) \phi / c^{2}\right] \omega_{12}(0)
$$

so that assuming $\bar{\omega}_{12}(0)=\omega_{12}(0)$

$$
\left[\bar{\omega}_{12}(\phi)-\omega_{12}(\phi)\right] / \omega_{12}(\phi)=3(1-f) \phi / c^{2} .
$$

If such a difference were observed, it might just as well be caused by a weak-equivalence-principle violation as by a $C P T$ violation. We could only distinguish the two possibilities by repeating the experiment at some other place where the value of $\phi$ was different. This might be accomplished by a variety of means, such as doing the experiment at the bottom and the top of a mountain. The simplest solution, however, would be just to do the experiment several times over a one year period, allowing the ellipticity of the earth's orbit to do the job of moving the apparatus through a change in the gravitational potential of the sun. The annual solar variation of $\phi / c^{2}$ is about $3 \times 10^{-10}$, about 300 times the variation due to the change in the earth's field between sea level and the top of Mount Everest. This null redshift experiment would then test the weak-equivalence principle to one part in $10^{9}$ if the experimental precision quoted

\footnotetext{
${ }^{27}$ Justified in the present case because we neglect spin effects and assume that $v / c \ll 1$.

${ }^{28}$ For a more detailed discussion of this point see Greenland (1997).
} 
above in connection with testing $C P T$ (one part in $10^{18}$ ) could be achieved. This is about the level of precision achieved by Eötvös.

\section{EXPERIMENTS WITH THE CERN AD}

Two important events for the future of antiproton physics occurred during the writing of this review-the approval of the CERN AD project (Berlin et al., 1996) and the initial phases of its experimental program, and the appearance on the horizon of a new $50-\mathrm{GeV}$ proton synchrotron, the Japan Hadron Facility (JHF-97-1, 1997). The latter has clearly identifiable possibilities for continuing and extending antiproton physics beyond the end of the AD's probable seven-year existence.

The approved AD experiments extend over most or all of this period and present problems which sometimes have no complete solutions and sometimes have several competing ones. Under these circumstances we can only summarize here the general direction these experiments will take, and refer the reader to publicly available documents presented to various experiment review committees and workshops for more detailed information.

\section{A. Cold antihydrogen: production and spectroscopy}

Two antihydrogen experiments are currently featured in the approved AD program (ATHENA, 1997; ATRAP, 1997). They have a number of similarities and differences in pursuit of a common goal. Both understandably approach this goal in a number of intermediate steps, after each of which branch points may occur according to what has been learned. Both also leave open to some extent the choice of recombination reactions among those mentioned in Sec. VII.B pending new experimental data during the first few years of AD operation. As far as either group can foresee over a 5-10 year period, the main milestones to be reached are (a) construction and testing of traps for positrons and antiprotons and testing of methods for bringing the trappedparticle clouds together, (b) studies of recombination reactions and the production of useful samples of antihydrogen atoms without trying to trap them, (c) capture of these in a neutral-atom trap and their cooling to $\mathrm{mK}$ temperature, and finally (d) spectroscopic measurements of increasing sensitivity and precision, with the ultimate goal of carrying out $C P T$ and WEP tests of the kind described in Secs. V and VII.

The experience gained in collecting and cooling bunched antiprotons in Penning-Malmberg traps during the last years of LEAR operation (Secs. IV.A.1-3) makes this the least problematical of all the technical questions to be addressed. Thus a sample of some $10^{7}$ antiprotons is taken by both groups as a nominal goal for a trap with parameters given in Table VII, following the achievement of a trapped sample of more than $10^{6}$ atoms from a single LEAR bunch by Holzscheiter et al. (1997). In arriving at this figure, it is assumed that some 100 AD bunches of $\sim 10^{7}$ antiprotons can be stacked in the trap with an efficiency of $0.5 \%$, cooling each bunch between one minute AD cycles.

Solutions to the more difficult problem of loading positrons into a trap from a continuous source have been described in Sec. VII.A. The ATRAP group is closely investigating the Schwinberg method, with the improvements expected from ${ }^{9} \mathrm{Be}^{+}$-ion damping, while ATHENA is studying the differentially pumped neutralgas damping method, which is, as we have seen (Greaves and Surko, 1997), capable of accumulating some $10^{8}$ positrons in a few minutes.

In the ATHENA case, the requirement of differential pumping means that two outer traps will first be filled with positrons and antiprotons, which will then be emptied into an inner nested Penning-Malmberg or combined Paul-Penning trap, now the reaction vessel. The ATRAP Collaboration (ATRAP, 1997) used the last days of LEAR operation to accumulate $\mathcal{O}\left(10^{6}\right)$ positrons and a somewhat smaller number of antiprotons directly into a nested trap. Although the two plasmas were brought into contact at $4 \mathrm{~K}$, no recombination was observed, for reasons which may not be understood until the experiment is repeated with the AD. Both collaborations plan to confine the neutral- $\bar{H}$ atoms in Ioffe traps (Sec. IV.C.1) at a later stage of experimentation, by superimposing the necessary radial and axial magnetic-field inhomogeneities on the reaction vessel, and to cool them by scattering of Lyman- $\alpha$ light.

Development of the many complex experimental systems briefly mentioned above will continue during the AD lead-in period. Evaluation of their performance requires a detailed study of recombination rates as a function of the particle density, number, and temperature in the clouds, as well as of the trap characteristics. For this purpose a diagnostic system that can detect both the location and the instant of annihilation of antiprotons, positrons, and antihydrogen atoms will be needed. Both groups, therefore, intend to surround their reaction vessels by segmented annihilation detectors along the lines of the wire chamber and silicon strip detectors more familiar in high-energy physics spectrometers, although on a much smaller scale.

It is only after all these phases have been accomplished that high-precision two-photon $1 S-2 S$ and other spectroscopic transitions will be attempted on the antihydrogen atom, and neither group seriously expects to be in a position to undertake any of these later measurements before the year 2002. To reach the ultimate goal of null comparisons of hydrogen and antihydrogen will take some years more, perhaps until the end of the AD's useful life. It is towards an eventual antiproton facility at the Japan Hadron Facility that the antihydrogen physicists may eventually have to direct their attention.

\section{B. Antiprotonic helium and protonium}

The existence of metastable antiprotonic helium atoms and the laser resonance technique developed in the past five years have opened up promising vistas for new 


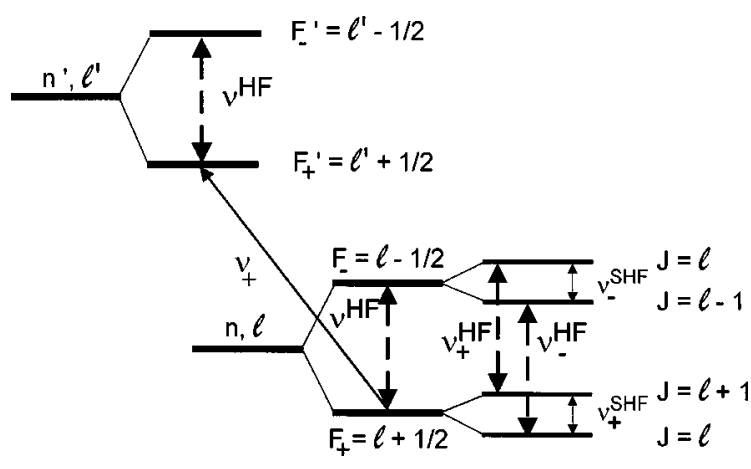

FIG. 24. Level diagram of the hyperfine-structure splitting in $\bar{p}$-He.

experiments on the $\bar{p}-\mathrm{He}^{++}$atom at the $\mathrm{AD}$, beginning in 1999 (ASACUSA, 1997). The interaction of the antiproton with the remaining electron is now well understood (Sec. IV.B.5): the energy eigenvalues for the $\bar{p}$ have now been calculated to a level of $\approx 5 \mathrm{ppm}$ (Korobov, 1996) by including relativistic terms in the electron's motion. Even closer agreement is obtained when the Lamb shift is taken into account (Elander and Yarevsky, 1997). A detailed study of the fine and hyperfine structure of the transitions between high-lying levels in $\bar{p}$-He could, for example, provide a much improved remeasurement of the magnetic moment of the antiproton.

The splitting comes about by the interaction of the magnetic moment,

$$
\left[g_{s}(\bar{p}) \overrightarrow{s_{\bar{p}}}+g_{l}(\bar{p}) \overrightarrow{l_{\bar{p}}}\right] \mu_{N},
$$

of the $\bar{p}$ with the nucleus and the remaining electron (hyperfine splitting). For ${ }^{4} \mathrm{He}$ the magnetic moment of the nucleus is zero.

The interaction is clearly dominated by the $\left(\vec{\ell}_{\bar{p}}, \vec{s}_{e}\right)$ interaction; the splitting $h \cdot \nu^{\mathrm{HF}}$ of a level $(n, \ell)$ into two levels $F_{ \pm}=\ell \pm 1 / 2$ by this interaction was calculated (Bakalov et al., 1996; Korobov, 1997) to be $\approx 52 \mu \mathrm{eV}$ for the $n=37, \ell=35$ level (see Fig. 24). An additional, much smaller super-hyperfine splitting into $J=F \pm 1 / 2 h \cdot \nu^{\mathrm{SHF}}$ $(\approx 0.7 \mu \mathrm{eV}$ for the same level), stems from the interaction of the $\bar{p}$ 's intrinsic magnetic moment with the electron spin and $\bar{p}$ angular momentum. This superhyperfine splitting should then determine $\mu_{\bar{p}}$.

An AD experiment with this aim should appear something like the following. Shoot a bunch of antiprotons into a He target and stop them there. Some of the $\bar{p}$ will be captured into the substates $\left(F_{-}=\ell-1 / 2, F_{+}=\ell\right.$ $+1 / 2)$ of a metastable level $(n, \ell)$ and will populate these substates (see Fig. 24) according to their multiplicity. By irradiation with laser light of resonance frequency $\nu_{+}$the state $F_{+}=\ell+1 / 2$ will be emptied to $F_{+}^{\prime}$, and the $\bar{p}$ will annihilate promptly after Auger transitions from this short-lived state. Apply a microwave pulse of suitable frequency and duration afterwards. Should the frequency correspond to one of the transition energies $\nu_{+}^{\mathrm{HF}}$ or $\nu_{-}^{\mathrm{HF}}$ (see Fig. 24), the population will change by induced transitions, oscillating between the levels with frequency $\nu_{0}=1 /(16 \pi \hbar) \cdot g \cdot \mu_{\mathrm{B}} \cdot B$ (where
$B=$ magnetic induction, $g=$ electron $g$ factor, and $\mu_{\mathrm{B}}$ $=$ Bohr magneton). Now adjust the microwave pulse duration so that it ends at the maximum population change, and irradiate the target again with laser light of frequency $\nu_{+}$to probe the new population of the $F_{+}$ states. If the microwave resonance condition is met, this population should have changed appreciably. From a comparison of $\Delta \nu=\nu_{-}^{\mathrm{HF}}-\nu_{+}^{\mathrm{HF}}(\approx 28 \mathrm{MHz})$ thus obtained with theory, the $\bar{p}$ magnetic moment may be deduced. As this method may not be very sensitive, a third, radiofrequency (rf) resonance can be used (ASACUSA, 1997) to determine $\nu^{\mathrm{SHF}}$ directly.

There are two prerequisites for this experiment to work:

(1) The difference between $\nu_{+}$and $\nu_{-}$has to be large enough to empty only the $F_{+}$state by laser irradiation (the bandwidth of the laser is-due to power and level broadening-finite). This is the case only for so-called "unfavored" transitions $\left(n, \ell \rightarrow n^{\prime}=n\right.$ $\left.+1, \ell^{\prime}=\ell-1\right)$ (Widmann et al., 1997).

(2) The microwave power has to be large enough to induce transitions between the hyperfine levels before all the antiprotons have annihilated or the empty state has been refilled from above. As an example, to invert the population of the $n=37, \ell$ $=35$ sublevels within $1 \mu \mathrm{s}$, a peak magnetic-field strength of $B=3 \times 10^{-4} \mathrm{~T}$ is necessary, which corresponds to a peak power of several tens of $\mathrm{kW}$.

The AD and trap and laser techniques might allow another appealing experiment to test $C P T$ in a purely baryonic environment, namely, the laser spectroscopy of protonium, $\bar{p} p$. This might be accomplished as follows:

Protonium atoms formed in a high- $n$, high- $\ell$ state should be long lived ( $\tau_{\text {rad }} \approx 1 \mu \mathrm{s}$ ), if collisions with other gas atoms are rare enough to suppress the Stark effect (this condition should be fulfilled at hydrogen pressures below $1 \mu$ bar $)$. Two laser pulses $(\lambda \approx 600 \mathrm{~nm})$ fired from opposite directions into the target should then induce two-photon resonant excitation of the $\bar{p}$ from $n \approx 40$ to $n \approx 50$. This two-photon excitation should efficiently suppress Doppler broadening and at the same time keep the linewidth small. The initially empty states around $n$ $=50$ will be populated when the resonance condition is met. A third laser pulse may then "ionize" the protonium atom from the $n \approx 50$ level (but not from the $n$ $\approx 40$ level), and the freed $\bar{p}$ may then be accelerated towards an electrode on which it will annihilate. The time coincidence of the annihilation products and the laser shots should signal the resonance condition.

Improving the bandwidth and absolute frequency calibration of the laser from the conditions during the LEAR era should make possible measurement of the antiprotonic atom's Rydberg energy with an accuracy exceeding the present value by orders of magnitude.

\section{CONCLUDING REMARKS-THE ROAD AHEAD}

The antiproton celebrated its fortieth birthday in November 1995 . This anniversary not only went largely un- 
noticed, it also heralded the beginning of the end of LEAR's useful life, the decision to discontinue this machine having been made in that year. Nevertheless, within a month or two of LEAR's formal closure ceremony in December 1996 it had been decided to construct a new antiproton facility, the Antiproton Decelerator (AD) literally out of the ashes of the old one.

Why has this phoenix arisen? No-one familiar with the funding crisis confronting basic science in the 1990s will be surprised that the decision was conditional on the AD's being financed from sources outside CERN. What a high-energy physicist might find astonishing, at least at first sight, is that it is being designed to provide samples of antiprotons that can be decelerated almost to rest. Our constant theme has been that it is not only interesting but essential to do this, and that while high-precision measurements of the static properties of the antiproton have improved consistently over the years, it is only recently that they have been interpretable as serious tests of $C P T$ invariance.

Two other important results from LEAR's declining years have been the first synthesis of antihydrogen atoms and the first spectroscopic studies done on an antiproton-containing atom, metastable $\bar{p} \mathrm{He}^{++} e^{-}$. At rest, the first of these provides an even better laboratory for studying $C P T$ invariance than the antiproton itself. In addition, it promises the first serious tests of the weak-equivalence principle in the antimatter domain; with antiprotons and positrons, only relatively lowprecision ballistic experiments seem possible, and none of them have as yet succeeded. Metastable $\bar{p} \mathrm{He}^{++} e^{-}$ has been the subject of the first-ever spectroscopic experiments on antiproton-containing atoms, with each successive year seeing increased experimental precision and scope going hand in hand with increased theoretical understanding, to arrive in the final year of LEAR's life at a measurement of the atom's hyperfine structure.

The dawn of the next century therefore holds out some enticing prospects for the study of the antiworld. By 2002, we may reasonably expect to see the synthesis of antihydrogen atoms at $\mathrm{mK}$ temperatures and the first laser-spectroscopic comparisons of hydrogen and antihydrogen. Concerning the antiprotonic helium atom, we must first note that the $C P T$-conjugate system is not available. While this makes null comparisons impossible, the atom is infinitely easier to produce and to study spectroscopically than is antihydrogen. By 2000 its fine and hyperfine structure should therefore be well understood and many of its visible-light transitions should have been measured to a few parts in $10^{8}$ and understood in terms of QED. We should also see the first steps being taken towards laser spectroscopy of metastable protonium. Finally, after the seven-year expected lifetime of $\mathrm{AD}$, it is certainly possible to envisage continuation of all this work at the Japan Hadron Facility.

We close this review by asking once more why we must do such experiments. In a 1995 talk entitled "Are matter and antimatter symmetric?" Lee (1996) looked back on the way the certainties of this century concerning $C, P, T$, and $C P$ symmetry turned in the end to doubts. His conclusion was that at least at the Planck scale of length (and maybe at a much larger scale of distance) the foundation of the $C P T$ theorem is also unsound, and that the symmetry between matter and antimatter therefore rests on experimental evidence alone.

If a man will begin with certainties, he shall end in doubts; but if he will be content to begin with doubts, he shall end in certainties. (Francis Bacon, 1605)

\section{ACKNOWLEDGMENTS}

The idea for this article originally came from A. Richter. During its preparation, the authors have benefitted from the assistance of so many of their colleagues that it would be impossible to mention them all by name. However, we should like to acknowledge the specific contributions of M. Charlton, P. T. Greenland, R. J. Hughes, D. Horváth, M. Mandelkern, D. Möhl, W. Pirkl, and G. Rouleau. Their constructive and careful reading of sections of the text resulted in many improvements, although they are in no way responsible for any errors. We also thank S. Geer for supplying information on the antiproton lifetime experiment, M. H. Holzscheiter, S. Maury, C. M. Surko, and M. Mandelkern for making original diagrams and unpublished data available, and T. Ypsilantis for putting the discovery of the antiproton into its historical context. It is a pleasure to acknowledge, too, the many insightful discussions at various times with $\mathrm{T}$. von Egidy, R. S. Hayano, and T. Yamazaki.

\section{REFERENCES}

AC Design Study Team, 1983, Design Study of an Antiproton Collector for the Antiproton Accumulator, CERN Yellow Report 83-10.

Adamo, A., et al., 1993, Phys. Rev. A 47, 4517.

Adams, C. S., and E. Riis, 1997, Prog. Quantum Electron. 21, 1.

Adelberger, E. G., B. R. Heckel, C. W. Stubbs, and Y. Su, 1991, Phys. Rev. Lett. 66, 850.

Adler, R., et al., 1997, in Proceedings of the Fourth Biennial Conference on Low Energy Antiproton Physics, edited by $\mathrm{H}$. Koch, M. Kunze, and K. Peters, Nucl. Phys. B, Proc. Suppl. 56A, 361.

Agnello, M., et al., 1995, Phys. Rev. Lett. 74, 371.

Ahlen, S. P., et al., 1988, Phys. Rev. Lett. 61, 145.

Akylas, V. R., 1978, Ph.D. thesis, preprint CALT-63-300 (California Institute of Technology, Pasadena, CA).

Akylas, V. R., and P. Vogel, 1978, Comput. Phys. Commun. 15, 291.

Andersen, H. H., and J. F. Ziegler, 1977, Hydrogen Stopping Powers and Ranges in all Elements (Pergamon, New York).

Andreae, T., W. König, R. Wynands, D. Leibfried, F. SchmidtKaler, C. Zimmermann, D. Meschede, and T. W. Hänsch, 1992, Phys. Rev. Lett. 69, 1923.

ASACUSA Collaboration proposal, 1997, CERN/SPSC 97-19, SPSC P-307.

Aschenauer, E. C., et al., 1992, CERN/SPSLC 92/28, SPSLC M494. 
ATHENA Collaboration proposal, 1997, CERN/SPSC 96-47, SPSC P-302; Memorandum CERN/SPSC 97-9, SPSC M-597. ATRAP Collaboration proposal, 1997, CERN/SPSC 97-8, SPSC P-306.

Bacher, R., P. Blüm, D. Gotta, K. Heitlinger, M. Schneider, J. Missimer, L. M. Simons, and K. Elsener, 1988, Phys. Rev. A 38, 4395.

Bacon, F., 1605, The Advancement of Learning I.i.3. A modern edition with an introduction by G. W. Kitchin can be found in the Everyman's Library series (Dent, London, 1954).

Baird, S., et al., 1997, in Proceedings of the Fourth Biennial Conference on Low Energy Antiproton Physics, edited by $\mathrm{H}$. Koch, M. Kunze, and K. Peters, Nucl. Phys. B, Proc. Suppl. 56A, 349.

Bakalov, D., I. V. Puzynin, T. P. Puzynina, and S. I. Vinitsky, 1996, Hyperfine Interact. 101/102, 487.

Barkas, W. H., W. Birnbaum, and F. M. Smith, 1956, Phys. Rev. 101, 778.

Barut, A. O., 1987, in Fundamental Symmetries, Proceedings of the First Course of the International School of Physics with Low-Energy Antiprotons, Erice, Italy, edited by P. Bloch, P. Pavlopoulos, and R. Klapisch (Plenum, New York), p. 227.

Baumann, J., R. Gähler, J. Kalus, and W. Mampe, 1988, Phys. Rev. D 37, 3107.

Baumann, J., R. Gähler, A. I. Ioffe, J. Kalus, and W. Mampe, 1989, Nucl. Instrum. Methods Phys. Res. A 284, 130.

Baur, G., et al., 1996, Phys. Lett. B 368, 251.

Bayfield, J. E., 1977, “Molecular Beams," in Encyclopaedia of Science and Technology (McGraw Hill, New York).

Beck, B. R., J. Fajans, and J. H. Malmberg, 1992, Phys. Rev. Lett. 68, 317.

Beck, B. R., J. Fajans, and J. H. Malmberg, 1996, Phys. Plasmas 3, 1250.

Beck, W. A., L. Wilets, and M. A. Alberg, 1993, Phys. Rev. A 48, 2779.

Becker-Szendy, R., et al., 1990, Phys. Rev. D 42, 2974.

Bell, J. S., 1955, Proc. R. Soc. London, Ser. A 231, 479.

Bell, M., et al., 1979, Phys. Lett. B 86, 215.

Bell, J. S., 1987, in Fundamental Symmetries, Proceedings of the First Course of the International School of Physics with Low Energy Antiprotons, Erice, Italy, edited by P. Bloch, P. Pavlopoulos, and R. Klapisch (Plenum, New York), p. 1.

Berlin, D., et al., 1996, Report CERN/PS 96-43(AR).

Bertulani, C. A., and G. Bauer, 1988, Phys. Rep. 163, 299.

Bethe, H. A., and E. E. Salpeter, 1977, Quantum Mechanics of One- and Two-electron Atoms (Plenum, New York), Chap. 12.

Beverini, N., et al., 1986a, CERN proposal PSCC-86.

Beverini, N., et al., 1986b, LANL report LA-UR-86-260 (unpublished).

Beverini, N., et al., 1988a, Phys. Rev. A 38, 233.

Beverini, N., et al., 1988b, Phys. Scr. T22, 238.

Blanford, G., D. C. Christian, K. Gollwitzer, M. Mandelkern, C. T. Munger, J. Schultz, and G. Zioulas, 1998a, Phys. Rev. Lett. 80, 3037.

Blanford, G., K. Gollwitzer, M. Mandelkern, J. Schultz, G. Takei, G. Zioulas, D. C. Christian, and C. T. Munger, 1998b, Phys. Rev. D 57, 6649.

Blanford, G., et al., 1998c, Proposal E903, Enrico Fermi National Accelerator Laboratory.

Bogomolov, E. A., et al., 1990, in Proceedings of the 21st International Cosmic Ray Conference, edited by R. J. Protheroe
(Department of Physics and Mathematical Sciences, University of Adelaide, 1990), Vol. 3, p. 288.

Bohnert, G., R. Decker, H. Pilkuhn, and H. G. Schlaile, 1986, Phys. Lett. B 174, 15.

Bondi, H., and R. A. Lyttleton, 1959, Proc. R. Soc. London Ser. A 252, 313.

Borie, E., 1983, Phys. Rev. A 28, 555.

Bregman, M., et al., 1978, Phys. Lett. B 78, 174.

Brown, D., G. Gabrielse, D. S. Hall, C. Heimann, W. Jhe, H. Kalinowsky, A. Khabbaz, T. Roach, and P. Yesley, 1997, in Proceedings of the Fourth Biennial Conference on Low Energy Antiproton Physics, Dinkelsbühl, 1996, edited by $\mathrm{H}$. Koch, M. Kunze, and K. Peters, Nucl. Phys. B, Proc. Suppl. 56A, 326.

Brown, L. S., and G. Gabrielse, 1986, Rev. Mod. Phys. 58, 233. Bruck, H., 1966, in Accelerateurs Circulaires des Particules (Institut Nationale des Sciences et Techniques Nucléaires, Saclay), p. 96.

Budker, G. I., 1966, in Proceedings of the Symposium Internationale sur les Anneaux de Collision, Saclay, edited by $\mathrm{H}$. Zyngier and E. Cremier-Alcan (Presses Universitaires de France, Saclay), p. II-1-1.

Budker, G. I., Ya. S. Derbenev, N. S. Dikansky, V. I. Kudelainen, I. N. Meshkov, V. V. Parkhomchuk, D. V. Pestrikov, B. N. Sukhina, and A. N. Skrinsky, 1975, IEEE Trans. Nucl. Sci. NS-22, 2093.

Budker, G. I., and A. N. Skrinsky, 1978, Sov. Phys. Usp. 21, 277. [See also Report of VAPP-NAP group to the International Conference on High Energy Particle Physics, Tbilisi (in English).]

Buffington, A., S. M. Schindler, and C. Pennypacker, 1981, Astrophys. J. 248, 1179.

Bunge, C. F., J. A. Barrientos, and A. V. Bunge, 1993, At. Data Nucl. Data Tables 53, 113.

Burbidge, G. R., and F. Hoyle, 1956, Nuovo Cimento 4, 558. Burbidge, G. R., and F. Hoyle, 1957, Astron. J. 62, 9.

Carbonell, J., and K. Protasov, 1993, Hyperfine Interact. 76, 327.

Caron, G., et al., 1978, Phys. Lett. B 77, 353.

Carosi, R., et al., 1990, Phys. Lett. B 237, 303.

Cesar, C. L., D. G. Fried, T. C. Killian, A. D. Polcyn, J. C. Sandberg, I. A. Yu, T. J. Greytak, D. Kleppner, and J. M. Doyle, 1996, Phys. Rev. Lett. 77, 255.

Cesar, C. L., 1997, in Proceedings of the International Workshop on Antimatter Gravity and Antihydrogen Spectroscopy, Sepino, 1996, Hyperfine Interact. 109, 293.

Chamberlain, O., E. Segrè, C. Wiegand, and T. Ypsilantis, 1955, Phys. Rev. 100, 947.

Chamberlain, O., et al., 1957, Nuovo Cimento 3, 447.

Charlton, M., J. Eades, D. Horváth, R. J. Hughes, and C. Zimmermann, 1994, Phys. Rep. 241, 65.

Charlton, M., and G. Laricchia, 1993, Hyperfine Interact. 76, 97.

Christian, D., G. Blanford, K. Gollwitzer, G. Zioulas, J. Schultz, M. Mandelkern, and C. Munger, 1997, Bull. Am. Phys. Soc. 42, 951.

Church, M. D., and J. P. Marriner, 1993, Annu. Rev. Nucl. Part. Sci. 43, 253.

Cohen, J. S., 1983, Phys. Rev. A 27, 167.

Cohen, J. S., 1997, Phys. Rev. A 56, 3583.

Cohen, A. G., D. B. Kaplan, and A. E. Nelson, 1993, Annu. Rev. Nucl. Part. Sci. 43, 27. 
Colladay, D., and V. A. Kostelecký, 1997, Phys. Rev. D 55, 6760.

Condon, E. U., and G. Breit, 1936, Phys. Rev. 49, 229.

Crowe, K. M., 1957, Nuovo Cimento 5, 541.

Daniel, H., 1975, Phys. Rev. Lett. 35, 1649.

Daniel, H., 1977, Nucl. Instrum. Methods 147, 297.

Daniel, H., 1979, Z. Phys. A 291, 29.

Daniel, H., 1980, Ann. Phys. (N.Y.) 129, 303.

Daniel, H., 1981, Z. Phys. A 302, 195.

Daniel, H., 1992, Nucl. Instrum. Methods Phys. Res. B 68, 459.

Darling, T. W., F. Rossi, G. I. Opat, and G. F. Moorhead, 1992,

Rev. Mod. Phys. 64, 237.

DeCecco, P., P. Hauser, D. Horváth, F. Kottmann, L. M. Simons, and D. Taqqu, 1997, Nucl. Instrum. Methods Phys. Res. A 394, 287.

de Raad, B., A. Minten, and E. Keil, 1966, CERN Yellow Report 66-21.

de Saint Simon, M., et al., 1995, Phys. Scr. T59, 406.

Dessler, A. J., F. C. Michel, H. E. Rorschach, and G. T. Trammell, 1968, Phys. Rev. 168, 737.

Deutch, B. I., 1992, Hyperfine Interact. 73, 175.

Dolinov, V. K., G. Ya. Korenman, I. V. Moskalenko, and V. P. Popov, 1989, Muon Catal. Fusion 4, 169.

Dylla, H. F., and J. G. King, 1973, Phys. Rev. A 7, 1224.

Eades, J., and L. M. Simons, 1989, Nucl. Instrum. Methods Phys. Res. A 278, 368.

Elander, N., and E. Yarevsky, 1997, Phys. Rev. A 55, 1855.

Ellis, J., N. E. Mavromatos, and D. V. Nanopoulos, 1992, Phys. Lett. B 293, 142.

Eötvös, v. R., D. Pekár, and E. Fekete, 1922, Ann. Phys. (Leipzig) 68, 11.

Ericson, T. E. O., and A. Richter, 1990, Europhys. Lett. 11, 295.

Essen, L., R. W. Donaldson, M. J. Bangham, and E. G. Hope, 1971, Nature (London) 229, 110.

Evans, L., E. Jones, and H. Koziol, 1989, in Proton-Antiproton Collider Physics, edited by G. Altarelli and L. DiLella (World Scientific, Singapore), p. 2.

Fano, U., 1953, Phys. Rev. 92, 328.

Fee, M. S., S. Chu, A. P. Mills Jr., R. J. Chichester, D. Zuckerman, E. D. Shaw, and K. Danzmann, 1993, Phys. Rev. A 48, 192.

Fermi, E., and E. Teller, 1947, Phys. Rev. 72, 399.

Gabrielse, G., X. Fei, K. Helmerson, S. L. Rolston, R. Tjoelker, T. A. Trainor, H. Kalinowsky, J. Haas, and W. Kells, 1986, Phys. Rev. Lett. 57, 2504.

Gabrielse, G., X. Fei, L. A. Orozco, R. Tjoelker, J. Haas, H. Kalinowsky, T. A. Trainor, and W. Kells, 1989, Phys. Rev. Lett. 63, 1360.

Gabrielse, G., X. Fei, L. A. Orozco, R. Tjoelker, J. Haas, H. Kalinowsky, T. A. Trainor, and W. Kells, 1990, Phys. Rev. Lett. 65, 1317.

Gabrielse, G., W. Jhe, D. Phillips, W. Quint, C. Tseng, L. Haarsma, K. Abdullah, J. Gröbner, H. Kalinowsky, 1993, Hyperfine Interact. 76, 81.

Gabrielse, G., D. Phillips, W. Quint, H. Kalinowsky, G. Rouleau, W. Jhe, 1995, Phys. Rev. Lett. 74, 3544.

Gabrielse, G., S. L. Rolston, and L. Haarsma, 1988, Hyperfine Interact. 44, 287.

Gähler, R., 1997, private communication.

Gähler, R., J. Kalus, and W. Mampe, 1982, Phys. Rev. D 25, 2887.
Gaisser, T. K., and R. K. Schaefer, 1992, Astrophys. J. 394, 174.

Galilei, G., 1638, Dialogues and Demonstrations Concerning Two New Sciences (Ed. Naz., Leyden), Vol. 8, 108.

Ganguli, S. N., P. K. Malhotra, R. Raghavan, A. Subramanian, and K. Sudhakar, 1978, Phys. Lett. B 74, 130.

Garcia-Muñoz, M., and J. A. Simpson, 1988, Space Sci. Rev. 46, 205.

Geer, S., J. Marriner, R. Ray, J. Streets, M. Lindgren, Th. Muller, J. Quackenbush, and T. Armstrong, 1994, Phys. Rev. Lett. 72, 1596.

Golden, R. L., S. Horan, B. G. Mauger, G. D. Badhwar, J. L. Lacy, S. A. Stephens, R. R. Daniel, and J. E. Zipse, 1979, Phys. Rev. Lett. 43, 1196.

Goldman, T., R. J. Hughes, and M. M. Nieto, 1987, Phys. Rev. D 36, 1254.

Golser, R., and D. Semrad, 1991, Phys. Rev. Lett. 66, 1831.

Good, M. L., 1961, Phys. Rev. 121, 311.

Greaves, R. G., and C. M. Surko, 1997, Phys. Plasmas 4, 1528.

Greaves, R. G., M. D. Tinkle, and C. M. Surko, 1994, Phys. Plasmas 1, 1439.

Greenland, P. T., 1997, Contemp. Phys. 38, 181.

Haarsma, L., K. Abdullah, and G. Gabrielse, 1995, Phys. Rev. Lett. 75, 806 .

Hall, D. S., and G. Gabrielse, 1996, Phys. Rev. Lett. 77, 1962. Hänsch, T., and C. Zimmermann, 1993, Hyperfine Interact. 76, 47.

Hartmann, F. J., 1990, in Electromagnetic Cascade and Chemistry of Exotic Atoms, edited by L. M. Simons, D. Horváth, and G. Torelli (Plenum, New York), p. 23.

Hauser, P., 1993, in Muonic Atoms and Molecules, edited by L.

A. Schaller and C. Petitjean (Birkhäuser, Basel), p. 67.

Hayano, R., et al., 1994, Phys. Rev. Lett. 73, 1485; Phys. Rev. Lett. 73, 3181(E).

Hayward, R. W., 1990, in Encyclopaedia of Physics, 2nd edition, edited by R. G. Lerner and G. L. Trigg (VCH Publishers, New York), p. 210.

Hellwig, H., R. F. C. Vessot, M. W. Levine, P. W. Zitzewitz, D. W. Allan, and D. J. Glaze, 1970, IEEE Trans Instrum. Meas. IM-19, 200.

Herring, C., and M. H. Nichols, 1949, Rev. Mod. Phys. 21, 185. Hof, M., et al., 1996, Astrophys. J. 467, L33.

Hojvat, C., and A. van Ginneken, 1983, Nucl. Instrum. Methods Phys. Res. 206, 67.

Holzscheiter, M. H., 1991, in Proceedings First Biennial Conference on Low Energy Antiproton Physics, edited by P. Carlson, A. Kerek, and S. Szilagy (World Scientific, Singapore), p. 437.

Holzscheiter, M. H., 1992, Phys. Scr. 46, 272.

Holzscheiter, M. H., X. Feng, T. Goldman, N. S. P. King, R. A. Lewis, M. M. Nieto, and G. A. Smith, 1996, Phys. Lett. A 214, 279.

Holzscheiter, M. H., et al., 1997, in Proceedings of the Fourth Biennial Conference on Low Energy Antiproton Physics, edited by H. Koch, M. Kunze, and K. Peters, Nucl. Phys. B, Proc. Suppl. 56A, 336.

$\mathrm{Hu}$, E., Y. Asano, M. Y. Chen, S. C. Cheng, G. Dugan, L. Lidofky, W. Patton, and G. A. Smith, 1975, Nucl. Phys. A 254, 403.

Hughes, R. J., 1993a, Contemp. Phys. 34, 177.

Hughes, R. J., 1993b, Nucl. Phys. A 558, 605c.

Hughes, R. J., and B. I. Deutch, 1992, Phys. Rev. Lett. 69, 579. 
Hughes, R. J., and M. H. Holzscheiter, 1992, J. Mod. Opt. 39, 263.

Hughes, V. W., 1957, Phys. Rev. 105, 170.

International Commission on Radiation Units and Measurements, 1993, Report 49: Stopping Powers and Ranges for Protons and Alpha Particles (ICRU, Bethesda, MD).

Iwasaki, M., et al., 1991, Phys. Rev. Lett. 67, 1246.

Iwasaki, M., 1994, private communication.

Jackson, G. P., 1997, in Proceedings of the International Workshop on Antimatter Gravity and Antihydrogen Spectroscopy, Sepino, May 1996, Hyperfine Interact. 109, 53.

Jackson, J. D., 1975, Classical Electrodynamics (Wiley, New York).

JHF-97-1, Proposal for Japan Hadron Facility, KEK Report 97-3, 1997.

Kabir, P. T., 1965, in Symmetries in Elementary Particle Physics, edited by A. Zichichi (Academic, New York), p. 11.

Kabir, P. T., 1995, in Proceedings of the Third Biennial Conference on Low Energy Antiproton Physics, edited by G. Kernel, P. Križan, and M. Mikuž (World Scientific, Singapore), p. 472.

Kalinowsky, H., 1993, Hyperfine Interact. 76, 73.

Kapchinsky, I. M., and V. A. Teplyakov, 1970, Prib. Tekh. Eksp. 2, 19 ("Linear accelerator with spatially homogeneous strong focusing," in Russian).

Kobayashi, M., and A. I. Sanda, 1992, Phys. Rev. Lett. 69, 3139.

Korenman, G. Ya., 1997, Preprint INP MSU 97-I/452, M. V. Lomonosov Moscow State University, D. V. Skobeltsyn Institute of Nuclear Physics, Moscow.

Korobov, V. I., 1996, Phys. Rev. A 54, R1749.

Korobov, V. I., 1997, in Proceedings of the Fourth Biennial Conference on Low Energy Antiproton Physics, edited by $\mathrm{H}$. Koch, M. Kunze, and K. Peters, Nucl. Phys. B, Proc. Suppl. 56A, 89.

Kreissl, A., et al., 1988, Z. Phys. C 37, 557.

Lagomarsino, V., G. Manuzio, and G. Testera, 1994, Phys. Rev. A 50, 977.

Lagomarsino, V., G. Manuzio, G. Testera, and M. H. Holzscheiter, 1996, Hyperfine Interact. 100, 153.

Lamb, W. E., and R. C. Retherford, 1950, Phys. Rev. 79, 549. LEAR Design Study Team, 1980, Design Study of a Facility for Experiments with Low Energy Antiprotons (LEAR), CERN Report CERN/PS/DL 80-7.

Lee, T. D., 1996, in The Discovery of Nuclear Antimatter, Conference Proceedings, Vol. 53, edited by L. Maiani and R. A. Ricci (Societa Italiana di Fisica, Bologna), p. 1.

Lee, T. D., R. Oehme, and C. N. Yang, 1957, Phys. Rev. 106, 340.

Lee, T. D., and C. N. Yang, 1956, Phys. Rev. 104, 254.

Leibfried, D., 1995, Ph.D. Thesis (University of Munich).

Leon, M., and J. H. Miller, 1977, Nucl. Phys. A 282, 461.

Leon, M., and R. Seki, 1974, Phys. Rev. Lett. 32, 132.

Leon, M., and R. Seki, 1977, Nucl. Phys. A 282, 445.

Leon, M., and R. Seki, 1978, Nucl. Phys. A 298, 333.

Li, G.-Z., R. Poggiani, G. Testera, and G. Werth, 1993, Hyperfine Interact. 76, 281.

Li, G.-Z., and G. Werth, 1992, Phys. Scr. 46, 587.

Lodi-Rizzini, E., and A. Zenoni, 1997, Nucl. Phys. News 7, 25. Lüders, G., 1954, K. Dan. Vidensk. Selsk. Mat. Fys. Medd. 28, 1.

Lüders, G., 1956, Naturwissenschaften 43, 121.

Lüders, G., 1957, Ann. Phys. (N.Y.) 2, 1.
Lüders, G., and B. Zumino, 1957, Phys. Rev. 106, 385.

Maas, F., et al., 1994, Phys. Lett. A 187, 247.

Maas, F., et al., 1995, Phys. Rev. A 52, 4266.

Malmberg, J. H., and J. S. de Grassie, 1975, Phys. Rev. Lett. 35, 577.

Malmberg, J. H., and C. F. Driscoll, 1980, Phys. Rev. Lett. 44, 654.

Mandelkern, M., et al., 1997, A Letter of Intent for a Continuation of E862: Measuring the Lamb Shift and Fine Structure of Antihydrogen, The E862 Collaboration, M. Mandelkern, contact person, Fermilab, Batavia, IL.

Marinelli, M., and G. Morpurgo, 1982, Phys. Rep. 85, 161.

Marinelli, M., and G. Morpurgo, 1984, Phys. Lett. B 137, 439.

Marion, J. B., and B. A. Zimmerman, 1967, Nucl. Instrum. Methods 51, 93.

Maury, S., 1997, in Proceedings of the International Workshop on Antimatter Gravity and Antihydrogen Spectroscopy, Sepino, 1996, Hyperfine Interact. 109, 43.

Merrison, J. P., H. Bluhme, J. Chevallier, B. I. Deutch, P. Hvelplund, L. V. Jørgensen, H. Knudsen, M. R. Poulsen, and M. Charlton, 1997, Phys. Rev. Lett. 78, 2728.

Meshkov, I. N., 1997a, Phys. Part. Nuclei 28, 198.

Meshkov, I. N., 1997b, in Proceedings of the International Workshop on Antimatter Gravity and Antihydrogen Spectroscopy, Sepino, 1996, Hyperfine Interact. 109, 33.

Meshkov, I. N., and A. N. Skrinsky, 1995, Report JINR-E9-95130 (JINR, Dubna).

Mikkelsen, H. H., 1992, Nucl. Instrum. Methods Phys. Res. B 69, 22.

Mills, A. P., and E. M. Gullikson, 1986, Appl. Phys. Lett. 49, 1121.

Mitchell, J. W., et al., 1996, Phys. Rev. Lett. 76, 3057.

Möhl, D., 1997, in Proceedings of the International Workshop on Antimatter Gravity and Antihydrogen Spectroscopy, Sepino, 1996, Hyperfine Interact. 109, 33.

Möhl, D., G. Petrucci, L. Thorndahl, and S. van der Meer, 1980, Phys. Rep. 58, 73.

Molière, G., 1947, Z. Naturforsch. 2a, 133; 1948, 3a, 78.

Møller, S. P., E. Uggerhøj, H. Bluhme, H. Knudsen, U. Mikkelsen, K. Paludan, and E. Morenzoni, 1997, Phys. Rev. A 56, 2930.

Morita, N., et al., 1994, Phys. Rev. Lett. 72, 1180.

Morrison, P., 1958, Am. J. Phys. 26, 358.

Mühlbauer, M., et al., 1996, Hyperfine Interact. 101/102, 607.

Munger, Ch. T., S. J. Brodsky, and I. Schmidt, 1993, Hyperfine Interact. 76, 175.

Munger, Ch. T., S. J. Brodsky, and I. Schmidt, 1994, Phys. Rev. D 49, 3228.

Nafe, J. E., and E. B. Nelson, 1947, Phys. Rev. 73, 718.

Nakamura, S. N., et al., 1994, Phys. Rev. A 49, 4457.

Nanopoulos, D. V., 1978, Reprint HUTP-78/A062 (Harvard University, Cambridge, MA).

Neumann, R., 1985, in Proceedings of the Workshop on Electron Cooling and Related Topics, ECOOL84, edited by $\mathrm{H}$. Poth, Report KfK 3846 (Kernforschungszentrum, Karlsruhe). Neumann, R., 1987, in Fundamental Symmetries, Proceedings of the International School of Physics with Low Energy Antiprotons, Erice, edited by R. Klapisch (Plenum, New York).

Neumann, R., 1988, Hyperfine Interact. 44, 305.

Neumann, R., H. Poth, A. Winnacker, and A. Wolf, 1983, Z. Phys. A 313, 253.

Nieto, M. M., and T. Goldman, 1991, Phys. Rep. 205, 221.

Okun, L. B., 1989, Phys. Today 42(6), 31. 
Okun, L. B., 1992, in Proceedings of the 29th Course on Physics at the Highest Energy and Luminosity (Plenum, New York), p. 1.

Orito, S., et al., 1995, in Proceedings of the 24th International Cosmic Ray Conference, Rome, Vol. 3, p. 76.

Pachucki, K., D. Leibfried, M. Weitz, A. Huber, W. König, and T. W. Hänsch, 1996, J. Phys. B 29, 177.

Park, D., 1958, Am. J. Phys. 26, 210.

Parkhomchuck, V. V., 1988, Hyperfine Interact. 44, 315.

Particle Data Group, Barnett, R. M., et al., 1996, Phys. Rev. D 54, 1.

Pauli, W., 1955, in Niels Bohr and the Development of Physics, edited by W. Pauli (Pergamon, New York), p. 30.

Payne, M. G., 1969, Phys. Rev. 185, 611.

Peaslee, D., 1996, in Proceedings of the Third International Conference on Nucleon-Antinucleon Physics, ITEP, Moscow 1995, Yad. Fiz. 59, 1672.

Petley, B. W., 1988, in The Fundamental Physical Constants and the Frontier of Measurement (Adam Hilger, Bristol/ Boston), p. 283.

Phillips, Th. J., 1995, in Proceedings of the Third Biennial Conference on Low Energy Antiproton Physics, edited by G. Kernel, P. Križan, and M. Mikuž (World Scientific, Singapore), p. 569.

Phillips, Th. J., 1997, in Proceedings of the International Workshop on Antimatter Gravity and Antihydrogen Spectroscopy, Hyperfine Interact. 109, 357.

Pilkuhn, H., and H. G. Schlaile, 1983, Phys. Rev. A 27, 657.

Pirkl, W., CERN PS Division, 1997, private communication.

Popper, K., 1959, The Logic of Scientific Discovery (Hutchinson, London).

Poth, H., et al., 1988, Hyperfine Interact. 44, 259.

Pritchard, D. E., 1983, Phys. Rev. Lett. 51, 1336.

Quint, W., and G. Gabrielse, 1993, Hyperfine Interact. 76, 379.

Roberson, P., et al., 1977, Phys. Rev. C 16, 1945.

Roberts, B. L., 1978, Phys. Rev. D 17, 358.

Roberts, B. L., et al., 1975, Phys. Rev. D 12, 1232.

Roll, P. G., R. Krotkov, and R. H. Dicke, 1964, Ann. Phys. (N.Y.) 26, 442.

Rolston, S. L., and G. Gabrielse, 1988, Hyperfine Interact. 44, 233.

Rook, J. R., 1970, Nucl. Phys. B 20, 14.

Russell, J. E., 1969, Phys. Rev. 188, 187.

Scheck, F., 1983, Leptons, Hadrons and Nuclei (NorthHolland, Amsterdam), p. 200.

Schempp, A., 1992, CERN Accelerator School, Report CERN 92-03, p. 522.

Schramm, U., et al., 1991, Phys. Rev. Lett. 67, 22.

Schwinberg, P. T., R. S. van Dyck, and H. Dehmelt, 1981, Phys. Lett. A 81, 119.

Segrè, E., 1958, Annu. Rev. Nucl. Sci. 8, 127.

Setija, I. D., H. G. C. Werij, O. J. Luiten, M. W. Reynolds, T. W. Hijmans, and J. T. M. Walraven, 1993, Phys. Rev. Lett. 70, 2257.

Seth, K., 1997, in Proceedings ot the Fourth Biennial Conference on Low Energy Antiproton Physics, Dinkelsbühl, 1996, edited by H. Koch, M. Kunze, and K. Peters, Nucl. Phys. B, Proc. Suppl. 56A, 385.

Shiekh, A. Y., 1997, in Proceedings of the International Workshop on Antimatter Gravity and Antihydrogen Spectroscopy, Sepino, 1996, Hyperfine Interact. 109, 105.

Shimamura, I., 1992, Phys. Rev. A 46, 3776.
Simons, L. M., 1993, Hyperfine Interact. 81, 253.

Stephens, S. A., and N. Finetti, 1996, Astron. Astrophys. 305, 367.

Streets, J., (APEX Collaboration), 1997, APS Spring Meeting. Streitmatter, R. E., S. J. Stochaj, J. F. Ormes, R. L. Golden, S. A. Stephens, T. Bowen, A. Moats, and J. Lloyd-Evans, 1989, Adv. Space Res. 9, No. 1265.

Thirring, W., 1961, Ann. Phys. (N.Y.) 16, 96.

Thirring, W., 1969, CERN notes.

Torii, H. A., et al., 1997, Nucl. Instrum. Methods Phys. Res. A 396, 257.

Udem, Th., A. Huber, B. Gross, J. Reichert, M. Prevedelli, M. Weitz, and T. W. Hänsch, 1997, Phys. Rev. Lett. 79, 2646.

van Dyck, R. S., Jr.,, P. B. Schwinberg, and H. G. Dehmelt, 1987, Phys. Rev. Lett. 59, 26.

Varelas, C., and J. Biersack, 1970, Nucl. Instrum. Methods 79, 213.

Vogel, P., 1974, At. Data Nucl. Data Tables 14, 599.

Vogel, P., P. K. Haff, V. Akylas, and A. Winther, 1975, Nucl. Phys. A 254, 445.

Vogel, P., A. Winther, and V. Akylas, 1977, Phys. Lett. B 70, 39.

Wald, R. M., 1980, Phys. Rev. D 21, 2742.

Walls, F. L., and H. G. Dehmelt, 1968, Phys. Rev. Lett. 21, 127.

Walz, J., S. B. Ross, C. Zimmermann, L. Ricci, M. Prevedelli, and T. W. Hänsch, 1995, Phys. Rev. Lett. 75, 3257.

Webber, W. R., and M. S. Potgieter, 1989, Astrophys. J. 344, 779.

Weiss, M., 1995, CERN Accelerator School, CERN 95-06, p. 959.

Weitz, M., A. Huber, F. Schmidt-Kaler, D. Leibfried, and T.

W. Hänsch, 1994, Phys. Rev. Lett. 72, 328.

Wess, J., 1988, Hyperfine Interact. 44, 1.

Wess, J., 1989, Nucl. Phys. B, Proc. Suppl. 8, 461.

West, D., 1958, Rep. Prog. Phys. 21, 271.

Widmann, E., et al., 1997, Phys. Lett. B 404, 15.

Wille, K., 1991, Rep. Prog. Phys. 54, 1005.

Wineland, D., C. Weimer, and J. Bollinger, 1993, Hyperfine Interact. 76, 115.

Winkler, P. F., D. Kleppner, T. Myint, and F. G. Walther, 1972, Phys. Rev. A 5, 83.

Witteborn, F. C., and W. M. Fairbank, 1967, Phys. Rev. Lett. 19, 1049.

Wolf, A., et al., 1986, in Low Energy Antimatter, Proceedings of the Madison Workshop 1985, edited by D. B. Cline (World Scientific, Singapore).

Wu, C. S., E. Ambler, R. W. Hayward, D. D. Hoppes, and R. P. Hudson, 1957, Phys. Rev. 105, 1413.

Yamazaki, T., et al., 1993, Nature (London) 361, 238.

Yamazaki, T., and K. Ohtsuki, 1992, Phys. Rev. A 45, 7782.

Yousif, F. B., P. van der Donk, Z. Kucherovsky, J. Reis, E. Brannen, J. B. A. Mitchell, and T. J. Morgan, 1991, Phys. Rev. Lett. 67, 26.

Ypsilantis, T., 1996, in The Discovery of Nuclear Antimatter, Conference Proceedings No. 53, edited by L. Maiani and R.

A. Ricci (Societa Italiana di Fisica, Bologna), p. 37.

Ypsilantis, T., 1997, private communication.

Zichichi, A., 1996, in The Discovery of Nuclear Antimatter, Conference Proceedings No. 53, edited by L. Maiani and R. A. Ricci (Societa Italiana di Fisica, Bologna), p. 123.

Ziegler, J. F., 1991, Code TRIM-91, IBM Research Division 28-0, Yorktown, NY 10598. 